\title{
Mode-Selective Vibrational Energy Transfer Dynamics in 1,3,5-Trinitroperhydro-1,3,5-Triazine (RDX) Thin Films
}

Neil Cole-Filipiak, Robert Knepper, Mitchell A. Wood, Krupa Ramasesha

Submitted date: 28/05/2021 P Posted date: 31/05/2021

Licence: CC BY-NC-ND 4.0

Citation information: Cole-Filipiak, Neil; Knepper, Robert; Wood, Mitchell A.; Ramasesha, Krupa (2021): Mode-Selective Vibrational Energy Transfer Dynamics in 1,3,5-Trinitroperhydro-1,3,5-Triazine (RDX) Thin Films. ChemRxiv. Preprint. https://doi.org/10.26434/chemrxiv.14691426.v1

Herein, we report on the sub-picosecond to sub-nanosecond vibrational energy transfer (VET) dynamics of the solid energetic material 1,3,5-trinitroperhydro-1,3,5-triazine (RDX) using broadband, ultrafast infrared transient absorption spectroscopy. Experiments reveal VET occurring on three distinct timescales: sub-picosecond, $5 \mathrm{ps}$, and $200 \mathrm{ps}$. The ultrafast appearance of signal at all probed modes in the mid-infrared suggests strong anharmonic coupling of all vibrations in the solid whereas the long-lived evolution demonstrates that VET is incomplete, and thus thermal equilibrium is not attained, even on the hundred picosecond timescale. Mode-selectivity of the longest dynamics suggests coupling of the $\mathrm{N}-\mathrm{N}$ and axial $\mathrm{NO}_{2}$ stretching modes with the long-lived, excited phonon bath.

File list (2)

RDX_VET_submission.pdf (1.66 MiB) view on ChemRxiv • download file 


\title{
Mode-Selective Vibrational Energy Transfer Dynamics in 1,3,5-Trinitroperhydro-1,3,5-Triazine (RDX) Thin Films
}

Neil C. Cole-Filipiak ${ }^{1}$, Robert Knepper ${ }^{2}$, Mitchell Wood ${ }^{2 *}$, Krupa Ramasesha ${ }^{1 *}$

${ }^{1}$ Combustion Research Facility, Sandia National Laboratories, Livermore, CA 94550, USA

${ }^{2}$ Sandia National Laboratories, Albuquerque, NM 87185, USA

*Authors to whom correspondence should be addressed: kramase@sandia.gov, mitwood@sandia.gov

\begin{abstract}
.
The coupling of inter- and intramolecular vibrations plays a critical role in initiating chemistry during the shock-to-detonation transition in energetic materials. Herein, we report on the subpicosecond to sub-nanosecond vibrational energy transfer (VET) dynamics of the solid energetic material 1,3,5-trinitroperhydro-1,3,5-triazine (RDX) using broadband, ultrafast infrared transient absorption spectroscopy. Experiments reveal VET occurring on three distinct timescales: subpicosecond, $5 \mathrm{ps}$, and $200 \mathrm{ps}$. The ultrafast appearance of signal at all probed modes in the midinfrared suggests strong anharmonic coupling of all vibrations in the solid whereas the long-lived evolution demonstrates that VET is incomplete, and thus thermal equilibrium is not attained, even on the hundred picosecond timescale. Density functional theory and classical molecular dynamics simulations provide valuable insights into the experimental observations, revealing compressioninsensitive timescales for the initial VET dynamics of high frequency vibrations and drastically extended relaxation times for low frequency phonon modes under compression. Mode-selectivity of the longest dynamics suggests coupling of the $\mathrm{N}-\mathrm{N}$ and axial $\mathrm{NO}_{2}$ stretching modes with the long-lived, excited phonon bath.
\end{abstract}

TOC Graphic:

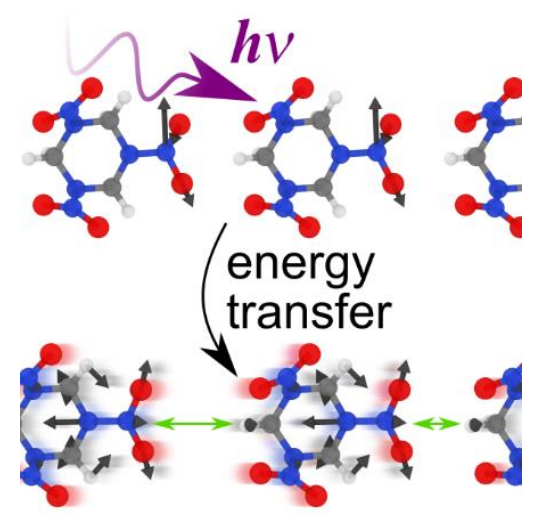

Keywords: ultrafast infrared spectroscopy; molecular crystals; energy transfer; energetic materials; RDX; HHTT; thin films; molecular dynamics; density functional theory; compression 


\section{INTRODUCTION}

Vibrational energy transfer (VET) via coupled molecular and lattice vibrations in energetic materials (EMs) is thought to influence the earliest chemical processes that lead to chemical bond cleavage and, ultimately, a detonation following a mechanical shock. Long-range interactionsmediated by low-frequency phonon modes - between the molecular constituents in an energetic solid play a critical role in VET, resulting in a complex interplay between inter- and intramolecular vibrations that is unique to molecular crystals. To explain the first steps in the shock-to-detonation transition, the multiphonon up-pumping model $^{1-3}$ posits that low frequency lattice modes (phonons) scatter into low-lying, molecular "doorway" states; thus multiple, successive scattering events partition more energy into successively higher energy vibrational modes until sufficient energy accumulates in vibrations associated with decomposition to begin the buildup to detonation. In an alternate and non-exclusive mechanism, the superposition of shock-excited lattice modes may directly couple to key reactive bonds with relatively few scattering events. ${ }^{4}$ Central to both models is whether or not the assumption of a rigid molecule ${ }^{5,6}$ holds true and the extent to which inter- and intramolecular vibrations are strongly coupled or may be treated separately, even when the lattice is compressed.

While there is rich literature investigating shockwave-induced processes in reactive and inert solids (see Refs. ${ }^{7-10}$ for recent examples), little experimental work exists ${ }^{11-14}$ on vibrational dynamics in solid, neat EMs that shed light on VET through strongly coupled, delocalized vibrational modes. Much of the existing VET research is performed in EM mimics or solvated

energetics (see Refs. ${ }^{15-17}$ for examples), where the importance of phonon-mediated long range interactions that form the basis for a complete picture of VET in EMs is not captured, leading to observed dynamics that are not representative of the dynamics in solid EMs. ${ }^{11,12}$ Furthermore, the 
molecular conformation of solvated energetics can be different from that in extended EM solids, ${ }^{12}$ resulting in differences in the nature of intermolecular interactions and vibrational coupling. Therefore, understanding the role of phonon-mediated interactions in guiding vibrational energy flow requires direct studies of ultrafast VET in solid EMs.

Given the broad, far- to mid-infrared, frequency range of vibrations in molecular crystals, VET investigations require ultrafast infrared spectroscopy to be coupled with broadband infrared pulses to derive a global view of VET dynamics. A recent study by our team used ultrafast broadband infrared spectroscopy and density functional theory to investigate VET in a pentaerythritol tetranitrate (PETN-I) thin film, revealing dynamics ranging from sub-picosecond to hundreds of picosecond timescales, and suggesting that VET is incomplete even up to $150 \mathrm{ps},{ }^{13}$ an unexpected finding given the typical timescales assumed for statistical energy partitioning. ${ }^{18}$ Unfortunately, due to the broad, overlapping vibrational transitions in PETN, we were unable to determine whether the long-lived VET process was due to persistent excitation of certain highfrequency vibrations or continued evolution of a non-thermal distribution of low frequency lattice modes that, in turn, perturbed ${ }^{6}$ the measured high-frequency vibrations. Regardless of the source of the long-time evolution, modes associated with the reactive nitrate ester $\left(\mathrm{RO}-\mathrm{NO}_{2}\right)$ stretch coordinate revealed some of the most dramatic spectral changes on the longest time scale.

In order to test if these long-time dynamics are anomalous and isolated to PETN, herein we study another molecular crystal, the secondary explosive 1,3,5-trinitroperhydro-1,3,5-triazine (RDX, also known as HHTT or cyclotrimethylenetrinitramine)—see Fig. 1 for an isolated molecular structure. Crystalline RDX has several polymorphs with $\alpha$-RDX as the most stable polymorph at room temperature and ambient pressure. In the $\alpha$-polymorph, the RDX ring takes on a chair conformer with two of the $\mathrm{NO}_{2}$ groups pointed axially and the third $\mathrm{NO}_{2}$ group oriented 
orthogonally along the equatorial direction ${ }^{19,20}$ (other polymorphs are characterized by different $\mathrm{NO}_{2}$ group orientations) with the crystalline unit cell qualitatively described as a quartet of dimers linked through adjacent $\mathrm{N} \cdots \mathrm{O}$ interactions. ${ }^{21,22}$ Cleavage of an $\mathrm{N}-\mathrm{N}$ bond and the production of various nitrogen-containing products (e.g. $\mathrm{NO}_{2}, \mathrm{HNO}, \mathrm{HONO}$ ) have been observed from thermal${ }^{23-25}$ and shock-excited ${ }^{26}$ samples and are thought be one of the first chemical steps in RDX initiation. Recent theoretical treatments of bond excitation ${ }^{27}$ and thermal conductivity ${ }^{28}$ indicate that RDX is a highly anharmonic molecular crystal where excited lattice modes are able to directly couple into the $\mathrm{N}-\mathrm{N}$ bond scission reaction coordinate; preliminary computational work by Chung and co-workers ${ }^{29}$ reports timescales of 0.16 and 5.6 ps for direct coupling and multiphonon uppumping to excite the $\mathrm{N}-\mathrm{N}$ bond, respectively. Indeed, several experimental and theoretical studies (Refs. ${ }^{30-32}$ for examples) have indicated anisotropic shock response in RDX, wherein it is hypothesized that select lattice distortions couple strongly to high frequency, "intramolecular" vibrations and also causes increased heating due to irreversible mechanical deformation on preferential slip planes..$^{31,33,34}$

To the best of our knowledge, there have been only two previous time-resolved, experimental studies of vibrational dynamics in solid RDX. The first study in single crystal RDX used anti-Stokes Raman spectroscopy to probe time-domain beating between different vibrational modes following broadband excitation below $1000 \mathrm{~cm}^{-1} \cdot{ }^{35}$ From the measured beat frequencies and known Raman transitions, two ring breathing motions at 466 and $672 \mathrm{~cm}^{-1}$ were postulated to play key roles in VET with decay timescales of $\sim 1$ ps. In the second study, Shi et al. employed ultrafast infrared pump-probe and two-dimensional infrared (2D IR) spectroscopy in the $1550 \mathrm{~cm}^{-}$ ${ }^{1}$ region corresponding to the axial and equatorial $\mathrm{NO}_{2}$ asymmetric stretching vibrations of microcrystalline RDX suspended in paraffin oil. ${ }^{12}$ Through measurements of vibrational relaxation 
times and the time evolution of a cross-peak assigned to the coupling of two $\mathrm{NO}_{2}$ stretching vibrations of $\alpha$-RDX dimers, two timescales were extracted and related to vibrational energy transfer using a phenomenological model: a $\sim 1.4$ ps timescale was assigned to intermolecular energy transfer between two neighboring $\mathrm{NO}_{2}$ stretching modes, whereas a 2-4.1 ps timescale was attributed to the vibrational relaxation of excited $\mathrm{NO}_{2}$ stretching modes to vibrations outside the probed frequency region. Due to the narrowband infrared pulses used in these experiments, only the $\mathrm{NO}_{2}$ stretching vibrations could be observed, and information regarding the role of other vibrations in VET could not be obtained.

In the current work, we present experimental and theoretical investigations of ultrafast, broadband VET dynamics in $\alpha$-RDX to understand the influence of chemical moieties on phononmediated energy transfer. Work presented here uses a plasma-based source of broadband infrared pulses to probe vibrational dynamics in RDX from $\sim 800 \mathrm{~cm}^{-1}$ to $1700 \mathrm{~cm}^{-1}$, and with $<300$ fs instrument response time, following narrowband infrared excitation of modes near $1530 \mathrm{~cm}^{-1}$ and $1260 \mathrm{~cm}^{-1}$. Our experimental observations are compared to VET pathways derived from density functional theory (DFT), as well as to the mode- and excitation-specific dynamics revealed by non-equilibrium molecular dynamics (MD) simulations. Both simulation methods also shed light on the response of the infrared spectrum to compression along different orientations, allowing us to draw additional insight into the role of intermolecular coupling in the observed dynamics.

As a brief aside on terminology, the terms "vibration" and "phonon" have been used interchangeably by some publications (see Ref. ${ }^{36}$ for example) or with strict separation of molecular and lattice motions-intra- and intermolecular modes, respectively—in a molecular crystal (as in Ref. ${ }^{18}$ for example). However, "intramolecular" modes may span a few tens of molecules ${ }^{11}$ and a clear distinction between RDX molecular vibrations and phonons or lattice 
vibrations appears to be inappropriate. ${ }^{27}$ Herein, frequencies observed in the experimental probe window will be generally referred to as "intramolecular" or "vibrations" and low frequency modes lying outside of this window will be referred to as "intermolecular," "phonon," or "lattice" modes; we separate intra- and intermolecular vibrations to convey an overall picture while noting that this distinction, particularly in the case of RDX as noted above, is not rigorous. In discussions of computational results, there is similarly no concrete way to define inter- and intramolecular vibrations and we will therefore use the experimental naming convention for consistency.

\section{METHODS}

\section{A. Experimental}

The RDX thin film samples were vapor-deposited in a bespoke high-vacuum system onto $1 \mathrm{~mm}$ thick, $25 \mathrm{~mm}$ diameter substrates of both $\mathrm{CaF}_{2}$ and BK-7 glass. Deposition was performed at a typical base pressure of $1 \times 10^{-6}$ torr using an effusion cell thermal deposition source to a nominal thickness of $\sim 3 \mu \mathrm{m}$. Immediately prior to deposition, both substrates were etched in situ using a Veeco Mark II Gridless End-Hall argon ion source to remove surface contaminants. Films on $\mathrm{CaF}_{2}$ substrates were used for the experiments described below while those on BK-7 substrates were used for microstructure characterization. Though the morphology of the deposited film strongly depends upon the surface energy of the substrate, ${ }^{37-39}$ the surface energy of clean $\mathrm{CaF}_{2}$ is similar to typical oxides ${ }^{40,41}$ and we expect the final morphologies to be comparable between substrates. Microstructure characterization was performed using scanning electron microscopy (SEM) to image the top surface and a fracture cross-section of films of each material. Samples were coated with $\sim 5 \mathrm{~nm}$ of iridium prior to imaging to mitigate surface charging. Images, shown in Fig. S1 in the Supporting Information (SI), were collected using a Zeiss GeminiSEM 300 operating with a $1.1 \mathrm{kV}$ accelerating voltage and using the secondary electron detector. For these 
thin films, $\alpha$ - RDX is expected to be the dominant polymorph; ${ }^{19,42}$ the FTIR spectrum shown in Fig. 1 supports this conclusion. ${ }^{43}$

The apparatus used for ultrafast broadband infrared spectroscopy has been previously described in detail. ${ }^{13,44}$ Briefly, the $\sim 50 \mathrm{fs}, 780 \mathrm{~nm}$ output from a commercial Ti:Sapphire laser is split into "pump" and "probe" arms, where the pump photoexcites the sample and the probe measures the pump-induced response. The pump arm of the laser output is first sent into a commercial optical parametric amplifier (OPA), whose signal and idler outputs are difference frequency mixed (DFM) and sent through a bandpass filter to generate tunable, narrowband midinfrared pulses that excite specific infrared transitions in the material. Two pump frequencies were used in the present experiments: i) $1530 \mathrm{~cm}^{-1}$ at $\sim 1.8 \mu \mathrm{J}$ pulse $\mathrm{s}^{-1}$ with a full-width-at-half-maximum (FWHM) of $150 \mathrm{~cm}^{-1}$ and ii) $1260 \mathrm{~cm}^{-1}\left(\sim 1.5 \mu \mathrm{J}\right.$ pulse $\left.\mathrm{p}^{-1}, 100 \mathrm{~cm}^{-1} \mathrm{FWHM}\right)$. A mechanical chopper wheel in the pump arm blocks alternating pump pulses to allow for shot-to-shot subtraction and measurement of pump-induced signal.

The probe arm of the laser output is used to generate a plasma which radiates light that spans visible to the terahertz frequencies, ${ }^{45}$ we select the mid-infrared portion by passing the plasma emission through a $0.5 \mathrm{~mm}$ thick Si wafer. These weaker broadband infrared (BBIR) pulses serve as the probe to monitor all IR-active vibrations within the detected bandwidth of 800-4000 $\mathrm{cm}^{-1}$. The pump and probe pulses are delayed with respect to each other using a motorized optical delay line for $\sim 330 \mathrm{ps}$ of total time-delay $(\Delta t)$, then focused and overlapped in the sample, and the transmitted probe pulse is sent into an infrared spectrometer and dispersed onto a 64-element, liquid nitrogen cooled $\mathrm{HgCdTe}$ array detector. Spectra were acquired with a 50 lines/mm grating for a spectral resolution of $\sim 15 \mathrm{~nm} /$ pixel. Multiple grating positions were required to record transients over the probe region of interest; the static absorption features of RDX-as measured 
by a commercial FTIR - were used to calibrate the reported frequencies at each spectrometer grating position. A $7.3 \mu \mathrm{m}$ long-pass filter was placed in the beam path prior to the detector aperture to minimize artifacts from higher order diffraction in the spectrometer at probe frequencies $<1370 \mathrm{~cm}^{-1}$. Experiments involving narrowband IR pump and broadband IR probe were performed with parallel relative polarizations of the pump and probe and orientational dynamics may contribute to the observed dynamics. ${ }^{46}$

The $1 / e^{2}$ focal diameters of the pump and probe beams at the sample position are $290 \mu \mathrm{m}$ (1530 $\mathrm{cm}^{-1}$ pump), $250 \mu \mathrm{m}\left(1260 \mathrm{~cm}^{-1}\right.$ pump), and $220 \mu \mathrm{m}$ (probe). Pump power studies were conducted using a half-wave plate and polarizer in the pump beam path. By monitoring the transient signal from a pumped band at $\Delta t=30 \mathrm{ps}$, the power dependence was measured at 1450 $\mathrm{cm}^{-1}$ and $1258 \mathrm{~cm}^{-1}$ to be $1.1 \pm 0.12$ and $1.2 \pm 0.16$ for the 1530 and $1260 \mathrm{~cm}^{-1}$ pump pulses, respectively. The two pump spectra are shown in Fig. 1; the $1530 \mathrm{~cm}^{-1}$ pump excites both the axial and equatorially oriented $\mathrm{NO}_{2}$ asymmetric stretching modes of $\alpha-\mathrm{RDX}$ and the $1260 \mathrm{~cm}^{-1}$ pump will excite multiple modes with $\mathrm{CH}_{2}$ bending, $\mathrm{N}-\mathrm{N}$ and $\mathrm{C}-\mathrm{N}-\mathrm{C}$ stretching, and $\mathrm{NO}_{2}$ bending/symmetric stretching character (see Fig. 1 and associated discussion). ${ }^{47}$ While the 1260 $\mathrm{cm}^{-1}$ pump is expected to only directly pump the two vibrational modes within its bandwidth, the $1530 \mathrm{~cm}^{-1}$ pump may also excite both an overtone of the mode at $780 \mathrm{~cm}^{-1}\left(\right.$ Ref. ${ }^{47}$ ) and possiblyvia two-photon excitation - the high frequency $\mathrm{C}-\mathrm{H}$ stretching modes ${ }^{47}$ around $3060 \mathrm{~cm}^{-1}$. Given the relatively low laser fluence used in the present work ( $c f$. Refs. ${ }^{11-13,17}$ ) and that absorption cross-sections for both two-photon and overtone absorption are likely small, other photoexcitation pathways are unlikely and we will assume photoexcitation of only the RDX fundamental vibrations within the $1530 \mathrm{~cm}^{-1}$ pump bandwidth. 
For transient infrared spectra where pump and probe frequencies are the same, the output from the OPA-DFM set-up is split into a 0.95/0.05 ratio, where the $95 \%$ arm serves as the pump and the 5\% arm serves as the probe. In order to minimize scatter of the pump beam at the sample from reaching the detector, these degenerate pump-probe experiments are performed with the pump polarization perpendicular to the probe polarization; a wire-grid polarizer, oriented along the probe polarization axis, is placed before the detector to minimize the detected pump light.

The location of maximum pump-probe temporal overlap $(\Delta t=0)$ was found by placing a $0.5 \mathrm{~mm}$ thick sample of Ge (100) or InSb (100) at the sample position. The temporal instrument response function (IRF) is assumed to have a Gaussian profile centered at $\Delta t=0$ with temporal FWHM given by the pump-probe cross-correlation in InSb. Cross-correlation widths with the 1530 $\mathrm{cm}^{-1}$ and $1260 \mathrm{~cm}^{-1}$ pump beams were approximately 200 and $300 \mathrm{fs}$, respectively, though we note that at least two photons of the $1260 \mathrm{~cm}^{-1}$ pump are required to excite the InSb bandgap. To minimize the effects of long-term drifts in laser power, time-delays between the pump and probe pulses were randomly sampled from a set list. Reported transient spectra are the average of repeated runs for a typical total of $\sim 10,000$ laser shots per difference spectrum.

Finally, linear infrared absorption spectra of RDX were taken at $24^{\circ} \mathrm{C}, 35^{\circ} \mathrm{C}$, and $37^{\circ} \mathrm{C}$ using a commercial FTIR spectrophotometer. Temperatures were chosen based on an upper limit temperature rise of approximately $10 \mathrm{~K}$ assuming $2 \mu \mathrm{J}$ of pump light is absorbed into a $3 \mu \mathrm{m}$ thick by $200 \mu \mathrm{m}$ diameter cylinder of RDX with density $1.8 \mathrm{~g} / \mathrm{cm}^{3}$ and $C_{\mathrm{p}}=1.28 \mathrm{~J} \mathrm{~g}^{-1} \mathrm{~K}^{-1} .48$

\section{B. Computational}

A combination of first-principles density functional theory (DFT) and classical molecular dynamics (MD) simulations were performed to complement the experimental VET work, each 
providing detail on VET pathways and timescales, respectively. All DFT calculations were performed within the Vienna Ab-Initio Simulation Package ${ }^{49-51}$ (VASP) while all classical MD simulations were performed utilizing the Large-scale Atomic/Molecular Massively Parallel Simulator (LAMMPS) software package.$^{52,53}$ A set of DFT calculations were performed in order to aid vibrational mode assignments, with the starting point being an equilibrated $\alpha$-RDX unit cell structure. ${ }^{19}$ We employ the generalized gradient approximation in the Perdew-Burke-Emzerhof form, ${ }^{54,55}$ a plane wave cutoff of $1250 \mathrm{eV}, 2 \times 3 \times 3$ Monkhosrt Pack k-point mesh $(0.0386 \times$ $0.0298 \times 0.0317 \AA^{-1}$ ), electronic smearing width of $0.2 \mathrm{eV},{ }^{56}$ and van der Waals corrections from Grimme. ${ }^{57}$ Each calculation is converged to an energy difference of $10^{-8} \mathrm{eV}$. Normal modes of vibration are calculated utilizing the finite displacement method (FDM) that is available in Phonopy ${ }^{58,59}$ and infrared active modes are identified by projecting a polarizability tensor onto each normal mode. ${ }^{60}$ Visualization of the mass-weighted, normal mode atomic displacements that accompanies each mode assignment utilized the OVITO software package. ${ }^{61}$ Anharmonic coupling between vibrations is calculated similarly through a finite displacement method only taking into account up to two-phonon scattering processes ${ }^{62}$ more details are available in our previous work on PETN. ${ }^{13}$

Conversely, molecular dynamics simulations are used to study the time evolution of VET following a well-prescribed pump excitation. Vibrational density of states and IR spectra are calculated at any finite temperature via a Fourier-transform of atomic velocities ${ }^{63}$ and the dipole autocorrelation function, ${ }^{64}$ respectively. Each of these spectra are collected over tens of picoseconds of trajectories, either as one continuous run or the summation of shorter-but numerous - statistically equivalent simulations. ${ }^{65}$ A velocity/dipole sampling interval of 5 fs yields a frequency resolution of $0.41 \mathrm{~cm}^{-1}$. The non-reactive interatomic potential from Bedrov et al. ${ }^{66}$ 
was employed after adjusting $\mathrm{N}-\mathrm{O}$ and $\mathrm{C}-\mathrm{H}$ bond strengths in order to make a better agreement to experimental IR spectra; see section S5 of the SI for further information.

It is important to stress that these MD simulations are classical in nature, meaning true light-matter interactions are not captured and thus we need alternate means to generate vibrational excitations in a controlled fashion. While the popular Langevin thermostat ${ }^{67}$ has no preferential control over the temperature of individual vibrations (only the simulation as a whole via an external "thermostat" bath), the random and dissipative force terms that are used to control temperature can be modified to have a time dependent magnitude; this modification is known as the Generalized Langevin Equations (GLE) ${ }^{68,69}$ Since only a select few modes will resonate with these timevarying thermostat oscillations, the remaining vibrations of the material will interact with the thermal bath and only see random noise terms as the base Langevin equations prescribe. The net result is a few vibrations at higher temperature than the rest of the system, which is precisely what is needed to study VET from MD. Dettori et al. ${ }^{70,71}$ have demonstrated these methods for a pumpprobe style simulation of hydrogen-bonded liquids and here we follow a similar protocol to selectively target vibrations of interest. Each of these GLE pumped vibrations emulate a Gaussian centered pump of width $33 \mathrm{~cm}^{-1}$, which act on a $3 \times 3 \times 3$ super-cell of RDX for 10 ps to establish a non-equipartition of vibrational kinetic energy; the pump is subsequently removed and spectra are collected in the micro-canonical ensemble. During both the pump and measurement period, the average temperature (and total kinetic energy) is conserved and therefore, by comparing spectra at an equivalent temperature, excitations and depopulations of modes due to the pumped mode and subsequent VET may be identified. Spectra are collected for a total of $100 \mathrm{ps}$; the end of this sampling period is taken as the presumed equilibrium (thermalized) spectra to which the earlier mode populations are normalized. With these normalized spectra, the temporal behavior about a 
small frequency window $\left(10 \mathrm{~cm}^{-1}\right)$ is fit to a mono-exponential decay function to extract the single mode relaxation time (SMRT).

\section{RESULTS AND ANALYSIS}

\section{A. Steady-State Results}
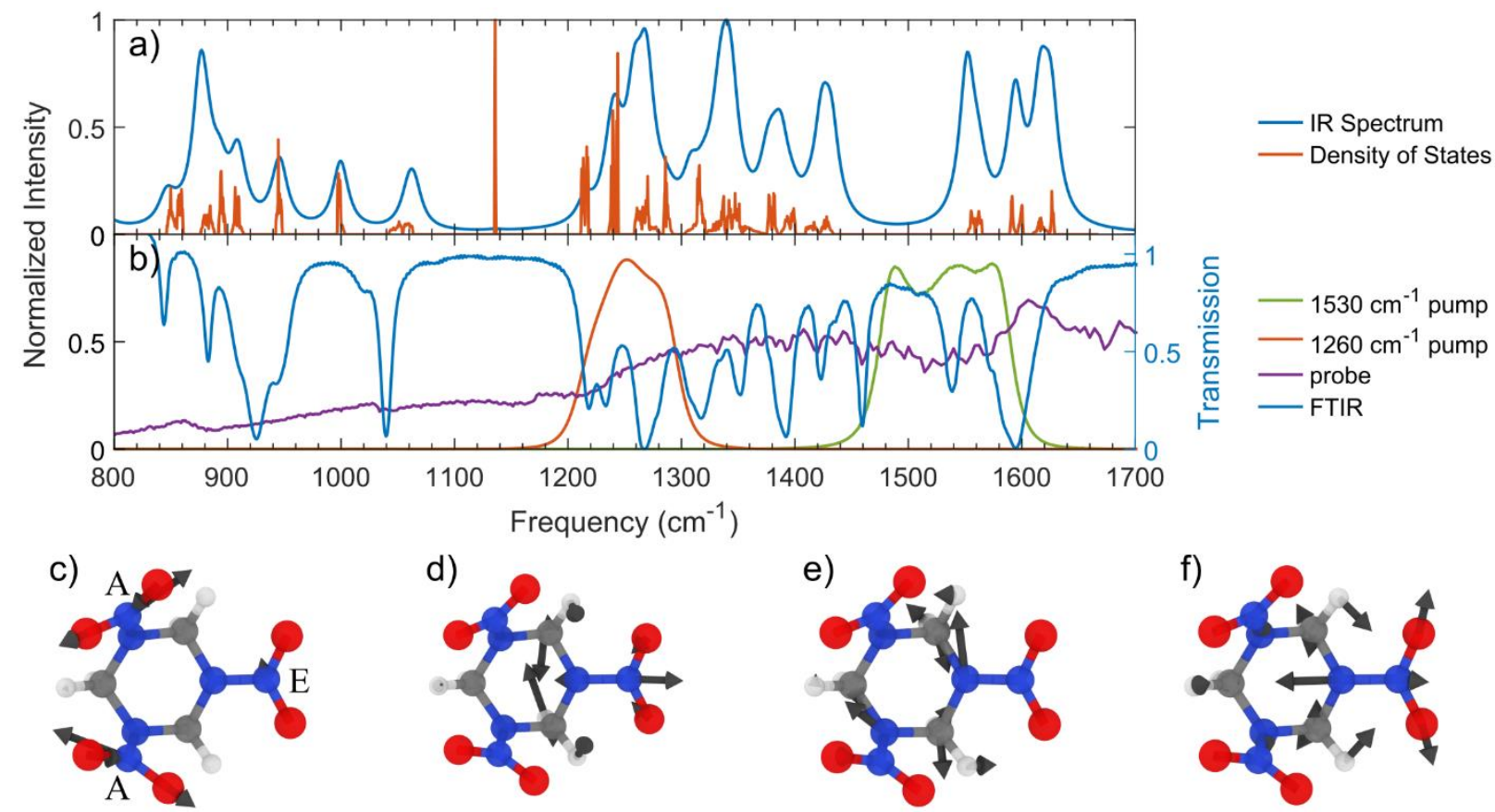

Figure 1. a) Calculated IR spectrum and density of states for $\alpha$-RDX using DFT. b) Experimental FTIR of the RDX thin film sample along with the pump and probe spectra. Representative crystalline $\alpha$-RDX vibrations with arrows showing mass-weighted atomic displacements for calculated frequencies of c) $1594 \mathrm{~cm}^{-1}$, d) $1432 \mathrm{~cm}^{-1}$, e) $1240 \mathrm{~cm}^{-1}$, and f) $1060 \mathrm{~cm}^{-1}$. Carbon atoms are gray, hydrogens are white, nitrogen atoms are blue, and oxygen atoms are red; axial and equatorial $\mathrm{NO}_{2}$ moieties are labeled $\mathrm{A}$ and $\mathrm{E}$, respectively. See Table $\mathrm{S} 1$ for additional modes.

We begin the results section by first examining the DFT calculated and experimentally measured static IR spectrum shown in Fig. 1 a) and b), respectively. The vibrational spectrum has been previously assigned in detail ${ }^{47}$ and the following is a brief summary of the main features with qualitative, "gas-phase"-like mode descriptions derived from our present calculations on crystalline $\alpha$-RDX; we note that these descriptions simplify the true motions in the extended crystal. The two bands at $\sim 1590$ and $1540 \mathrm{~cm}^{-1}$ correspond to the asymmetric stretch (as) of the 
two axial (ax) and one equatorial (eq) $\mathrm{NO}_{2}$ moieties, respectively - see Fig. $1 \mathrm{c}$ ) for an example as $-\mathrm{NO}_{2}{ }^{(\mathrm{ax})}$ vibration. Multiple peaks in the $1260-1480 \mathrm{~cm}^{-1}$ range correspond to the $\mathrm{CH}_{2}$ bending, twisting, and scissoring motions mixed with $\mathrm{N}-\mathrm{N}$ stretching character; for some modes the $\mathrm{NO}_{2}$ groups show bending and symmetric stretching (ss) motion, though the distinction between the two is less intuitive when accompanied by N-N stretch excitation. The band at $\sim 1270 \mathrm{~cm}^{-1}$ appears to be the $\mathrm{N}-\mathrm{N}^{(\mathrm{eq})}$ stretching and $\mathrm{CH}_{2}$ bending motion with a mix of $\mathrm{NO}_{2}$ bending/ss character. The next three peaks at 1040,1220 , and $1230 \mathrm{~cm}^{-1}$ are the ring $\mathrm{N}-\mathrm{C}-\mathrm{N}$ and $\mathrm{C}-\mathrm{N}-\mathrm{C}$ stretching modes which again contain $\mathrm{N}-\mathrm{N}$ stretching, $\mathrm{CH}_{2}$ twist or bend, and some $\mathrm{NO}_{2}$ bend/ss character; see example in Fig. $1 \mathrm{f}$ ). The peak at $945 \mathrm{~cm}^{-1}$ is composed of $\mathrm{N}-\mathrm{N}$ stretching, $\mathrm{CH}_{2}$ wag, $\mathrm{NO}_{2}$ bending, and some ring breathing character while the large band centered at $930 \mathrm{~cm}^{-1}$ may be described as ring distortion, $\mathrm{CH}_{2}$ wag, and $\mathrm{NO}_{2}$ bend. Finally, the lowest frequency peaks in our observation window at 840 and $880 \mathrm{~cm}^{-1}$ are due to in- and out-of-plane ring deformations, respectively, mixed with $\mathrm{C}-\mathrm{N}$ and $\mathrm{N}-\mathrm{N}$ stretch, $\mathrm{CH}_{2}$ wag, and $\mathrm{NO}_{2}$ bending motions. Three IR-active, lower frequency modes outside of our probe spectral range have been previously assigned ${ }^{47}$ as ring $\mathrm{N}-\mathrm{C}-\mathrm{N}$ stretching $\left(780 \mathrm{~cm}^{-1}\right)$ and in-plane ring bending modes $\left(600\right.$ and $\left.590 \mathrm{~cm}^{-1}\right)$. The calculated IR spectrum is in good, qualitative agreement with the experimental IR features at high frequencies $\left(>1200 \mathrm{~cm}^{-1}\right)$. At lower frequencies, the approximation of using a single unit cell for the FDM becomes more apparent as the predicted normal modes involve larger amplitude deformations (ring bending, intermolecular motions); peaks at $<1050 \mathrm{~cm}^{-1}$ are noticeably shifted by tens of wavenumber with some regions $\left(e . g . \sim 925 \mathrm{~cm}^{-1}\right)$ in disagreement with experiment. These finite size effects are not easily overcome due to computational cost constraints: the unit cell of $\alpha$-RDX already contains 168 atoms and two-phonon scattering predictions (anharmonic expansion of FDM, see below) require 51,642 unique DFT calculations. 
Predictions of two-phonon density of states in RDX, shown in Fig. S2, count the number of possible two-phonon collisions (both promotion and decay) into each frequency. The plots show the change in scattering rates from a thermally occupied phonon population for a series of pumped modes each approximated as a Gaussian addition to the Bose-Einstein population at a prescribed frequency, henceforth called $\Delta 2 \mathrm{PhDoS}$. In general, the $\Delta 2 \mathrm{PhDoS}$ show dense spectra of possible collisions that do not strongly depend on the pumped mode, indicating that each pumped mode may potentially couple to a plethora of modes, including the low-frequency phonon bath. We note that the $\Delta 2 \mathrm{PhDoS}$ will predict scattering events into frequencies that are not native vibrations of the material, therefore the predicted IR spectrum is superimposed on these data.

\section{B. Time-Dependent Results}

Frequency-resolved transient absorption spectra for both pump frequencies are presented in Figures 2 and 3. Example transient spectra at select time-delays are shown in Fig. 4; additional transient spectra may be found in Figures S10 and S12. For the degenerate pump-probe experiments-Figures 2 a), $3 \mathrm{c}$ ), and $3 \mathrm{~d}$ )—significant oscillations due to pump-probe interference were present in the raw data (see Fig. S13). To minimize these oscillations, these data were filtered using an established method ${ }^{72,73}$ for removing time- and frequency-dependent oscillations due to perturbed free-induction decay (PFID); $;{ }^{747}$ the resulting filtered transients are shown in Figs. 2 and 3. Briefly, the filtering procedure applies a damping function to the two-dimensional Fouriertransform of the transient signal to remove signal contributions that lie outside the time-bandwidth limit. Unfortunately, particularly for excitation at $1260 \mathrm{~cm}^{-1}$, these oscillations were large in amplitude and varied too slowly for the filter to completely remove.

The transient behavior of RDX is qualitatively similar for both pump frequencies and, except where noted, the following descriptions will apply to all transient results. To avoid the 
interpretation of coherent artifacts, ${ }^{74-76}$ we will limit our analysis to signal occurring for $\Delta t \geq 0.3$ ps. By $0.3 \mathrm{ps}$, the transient spectra show a negative change in absorbance due to stimulated emission or loss of ground state absorption (henceforth referred to as "bleach") from all vibrational modes across the probed spectral range. Each bleach feature is paired with a positive transient absorption (TA) signal arising from the new, pump-induced vibrational transitions. Such prompt appearance of signal across the spectrum suggests strong anharmonic coupling between all vibrational modes of RDX. Following the sub- 0.3 ps appearance, these bleach/TA couplets typically show an initial decay over the first ps, followed by a few-ps evolution until $\Delta t \approx 10 \mathrm{ps}$ where the spectra show hallmarks of vibrational relaxation (see below). Finally, the transient spectral features at select vibrational modes intensify over the next several hundred ps.

To help guide the discussion of spectral evolution and RDX dynamics, the Glotaran software package ${ }^{77,78}$ was used to extract the time-dependence of spectral features using global lifetime analysis with a sequential kinetic model. For both pump frequencies, the best fits were achieved with five time constants ( $5 \tau$ model); fit parameters are summarized in Table 1, example fits in the $1560 \mathrm{~cm}^{-1}$ spectral region are shown in Fig. 5, and fit residuals and evolution associated difference spectra may be found in the SI. The first time constant was fixed at $10 \mathrm{fs}$ to account for remaining coherent artifacts near $\Delta t=0$. Similarly, the final time constant was fixed at $10^{6} \mathrm{ps}$ to account for the persistent signal extending beyond the temporal observation window. Due to uncertainties in the accurate location of $\Delta t=0$ at every probed frequency and-particularly for the two regions where pump and probe frequencies are degenerate-residual pump interference, the $\sim 0.5-0.9$ ps behavior described by $\tau_{2}$ in Table 1 will be referred to as "sub-ps."

To help determine which modes contribute to the sub-ps time constant, fits were also performed omitting the first floating time constant (i.e. $\tau_{2}$ in Table 1) and fixing the remaining four 
at their optimized values ( $4 \tau$ model). The reduced fit quality from the $4 \tau$ model - compare the two fits in Fig. 5 for examples — highlights spectral regions which require the sub-ps time constant; we note that neither model appears to adequately describe the sub-ps $a s-\mathrm{NO}_{2}{ }^{(\mathrm{eq})}$ behavior following excitation at $1260 \mathrm{~cm}^{-1}$. At both pump frequencies, the sub-ps time constant appears to be largely occurring at $1540 \mathrm{~cm}^{-1}$ and, as shown in Fig. S19, around $\sim 930 \mathrm{~cm}^{-1}$. The sub-ps time constant also is needed near 1260 and $1040 \mathrm{~cm}^{-1}$ following the $1530 \mathrm{~cm}^{-1}$ pump, and near 1395 and $1460 \mathrm{~cm}^{-1}$ following the $1260 \mathrm{~cm}^{-1}$ pump; residual $1260 \mathrm{~cm}^{-1}$ pump interference shown in Fig. $3 \mathrm{c}$ ) and d) makes it difficult to assess any sub-ps behavior in this spectral region. For these lower frequencies following the $1530 \mathrm{~cm}^{-1}$ pump, the $4 \tau$ model fit appears to decay faster than the actual spectral response, possibly suggesting competing growth and decay dynamics. Conversely, following excitation at $1260 \mathrm{~cm}^{-1}$ the $4 \tau$ model provides a reasonable fit around $1040 \mathrm{~cm}^{-1}$ but does not appear to decay fast enough at lower frequencies. Indeed, the marked difference in initial spectral shape at $\sim 930 \mathrm{~cm}^{-1}$ for the two pump frequencies - compare Fig. $4 \mathrm{c}$ ) and f)—suggests a different pathway for the fastest VET steps. Finally, we note that our sub-ps fit time constants from the $5 \tau$ model are similar to both the coherence dephasing ( $0.48 \mathrm{ps})$ and intermolecular energy transfer $(\sim 1.4 \mathrm{ps})$ time constants reported by Shi et al. ${ }^{12}$

Following the sub-ps spectral evolution outlined above, a 5 ps time constant describes multiple line shape changes that reflect a broadening and shifting of all infrared peaks, akin to what is expected upon an increase in temperature (e.g. Refs. ${ }^{36,79,80}$ ). As can be seen in Figures 2 , 3 and 4 , the changes observed include loss of peak intensity, loss of TA peaks $\left(e . g .1380 \mathrm{~cm}^{-1}\right)$, shifts of peak frequencies (e.g. 900-960 $\mathrm{cm}^{-1}$ ), and the appearance of positive signal at frequencies higher than the existing negative features (e.g. 1050 and $\left.\sim 1325 \mathrm{~cm}^{-1}\right)$. Our results reveal that this timescale reflects a global relaxation of all probed vibrations towards transient spectra that 
qualitatively resemble the thermal difference infrared spectrum (ThDIR; i.e. the change in linear IR absorption between two different sample temperatures) shown in Fig. S14. The 5 ps time constant is in excellent agreement with the previous transient infrared spectroscopy studies in RDX microcrystals ${ }^{12}$ (4.11 ps for the $a s-\mathrm{NO}_{2}{ }^{(\mathrm{eq})}$ stretch) and dilute solutions of $\mathrm{RDX}^{12,17}(\sim 5-6$ ps for the $a s-\mathrm{NO}_{2}$ stretching mode), which assigned this time constant to "vibrational relaxation" of the photoexcited $\mathrm{NO}_{2}$ stretch.

Finally, the transient behavior of select IR transitions reveal a $\sim 200$ ps increase in signal intensity with minimal shifts to the frequencies, suggesting that VET is incomplete by the longest time-delay of $\Delta t=300 \mathrm{ps}$. These long-time changes are most obvious at $\sim 1590 \mathrm{~cm}^{-1}\left(\right.$ as $-\mathrm{NO}_{2}{ }^{\text {(ax) }}$ stretch), $1460 \mathrm{~cm}^{-1}\left(\mathrm{CH}_{2}\right.$ scissor, $\mathrm{N}-\mathrm{N}$ stretch, $\mathrm{NO}_{2}$ bend $\left./ s s\right), 1350 \mathrm{~cm}^{-1}\left(\mathrm{CH}_{2}\right.$ scissor and bend, $\mathrm{N}-$ $\mathrm{N}$ stretch), $\sim 1270 \mathrm{~cm}^{-1}\left(\mathrm{~N}-\mathrm{N}^{(\mathrm{eq})}\right.$ stretch, $\mathrm{CH}_{2}$ bend, $\mathrm{NO}_{2}$ bend/ss), $1220 \mathrm{~cm}^{-1}$ (ring $\mathrm{C}-\mathrm{N}-\mathrm{C}$ stretch, $\mathrm{CH}_{2}$ bend), $1040 \mathrm{~cm}^{-1}$ (N-N ${ }^{(\mathrm{eq})}$ stretch, $\mathrm{NO}_{2}$ bend/ss, ring $\mathrm{C}-\mathrm{N}-\mathrm{C}$ stretch, $\mathrm{CH}_{2}$ twist and bend), and $\sim 945 \mathrm{~cm}^{-1}$ (N-N stretch, $\mathrm{CH}_{2}$ wag, $\mathrm{NO}_{2}$ bend, ring distortion) for both pump frequencies. Following excitation at $1260 \mathrm{~cm}^{-1}$, the $200 \mathrm{ps}$ evolution is also noticeable at $880 \mathrm{~cm}^{-1}$ (out-of-plane ring distortion, $\mathrm{CH}_{2}$ wag, $\mathrm{N}-\mathrm{N}$ stretch, $\mathrm{C}-\mathrm{N}$ stretch, $\mathrm{NO}_{2}$ bend) and possibly $1320 \mathrm{~cm}^{-1}\left(\mathrm{CH}_{2}\right.$ scissor, $\mathrm{CH}_{2}$ bend, $\mathrm{N}-\mathrm{N}$ stretch), though the apparent pump-dependence is potentially attributable to the differences in signal-to-noise levels and the relative intensity of a neighboring positive feature at $\sim 1330 \mathrm{~cm}^{-1}$ — compare Figs. S10 b) and S12 c)—respectively. To highlight these longtime changes, single-frequency transients of the above frequency set may be found in Figures 5 , S19, S20, and S21. The remaining IR bands show little to no evolution after the 5 ps time constant; indeed, as shown in Fig. 5, there is a clear difference in the long-time evolution of the as- $\mathrm{NO}_{2}{ }^{(\mathrm{ax})}$ and $a s-\mathrm{NO}_{2}{ }^{(\mathrm{eq})}$ stretching modes. The mode-selectivity of these long-time dynamics may indicate specific, vibrational modes that directly couple to the low frequency lattice motions. ${ }^{4,27}$ 
Table 1. Time constants (ps) from the global lifetime analysis of the transient data. Reported bounds are the standard error of the fit.

\begin{tabular}{|c|c|c|c|c|c|}
\hline Pump Freq. & $\boldsymbol{\tau}_{\mathbf{1}}$ & $\boldsymbol{\tau}_{\mathbf{2}}$ & $\boldsymbol{\tau}_{\mathbf{3}}$ & $\boldsymbol{\tau}_{\mathbf{4}}$ & $\boldsymbol{\tau}_{\mathbf{5}}$ \\
\hline $1530 \mathrm{~cm}^{-1}$ & 0.01 (fixed) & $0.93 \pm 0.03$ & $5.28 \pm 0.07$ & $190 \pm 20$ & $10^{6}$ (fixed) \\
\hline $1260 \mathrm{~cm}^{-1}$ & 0.01 (fixed) & $0.46 \pm 0.02$ & $5.03 \pm 0.07$ & $260 \pm 30$ & $10^{6}$ (fixed) \\
\hline $\begin{array}{c}\text { Reference in } \\
\text { text }\end{array}$ & sub-0.3 ps & sub-ps & $5 \mathrm{ps}$ & $\sim 200 \mathrm{ps}$ & \\
\hline
\end{tabular}
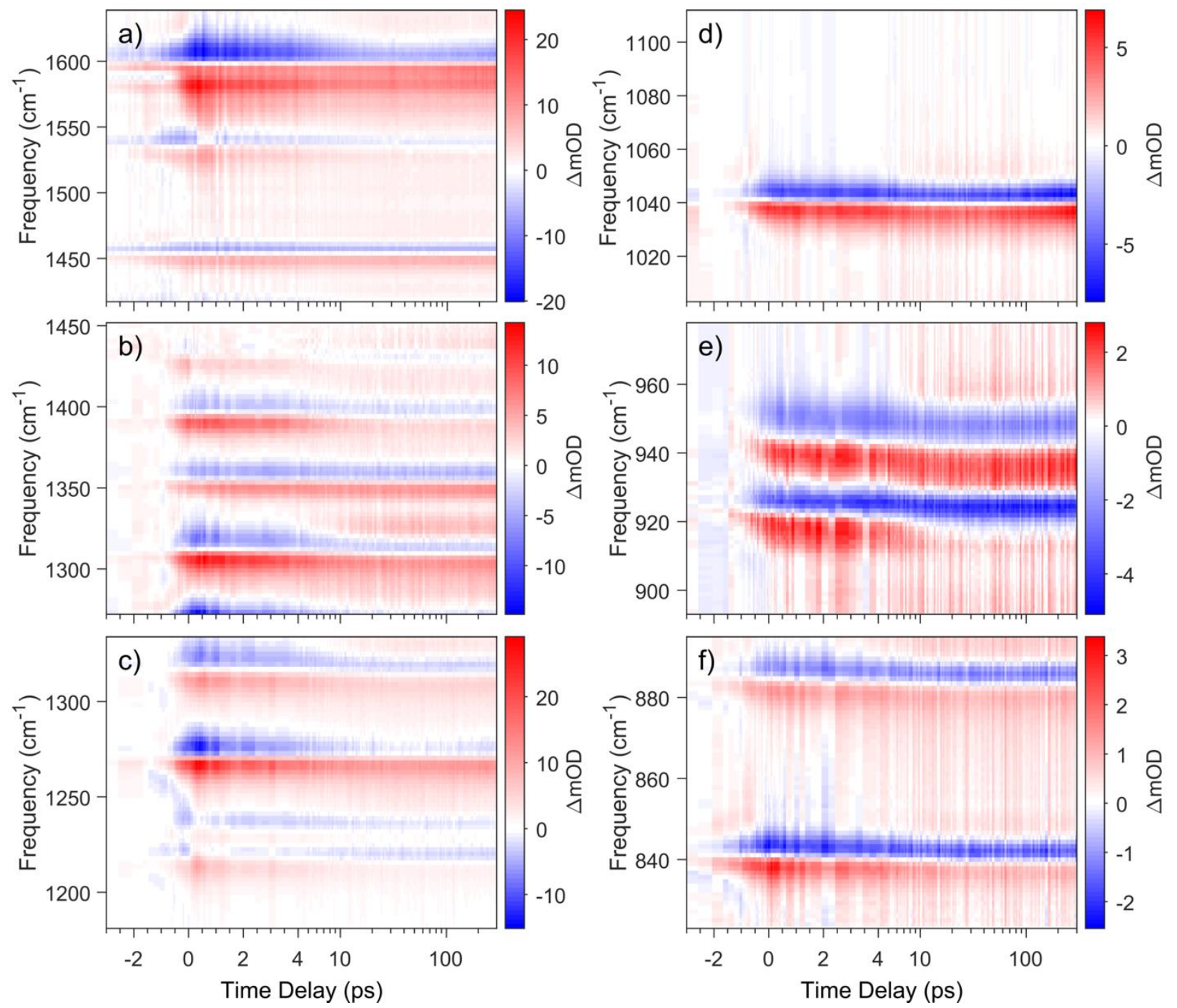

Figure 2. Transient spectra following excitation at $1530 \mathrm{~cm}^{-1}$ at each spectrometer grating positions. Time-delays are linear up to $4 \mathrm{ps}$ and logarithmic thereafter. The oscillations near $\Delta t=$ 0 in a) are the residual pump-probe interference pattern that was not fully removed by filtering; see text for additional details. See Fig. S9 for an alternate colormap. 

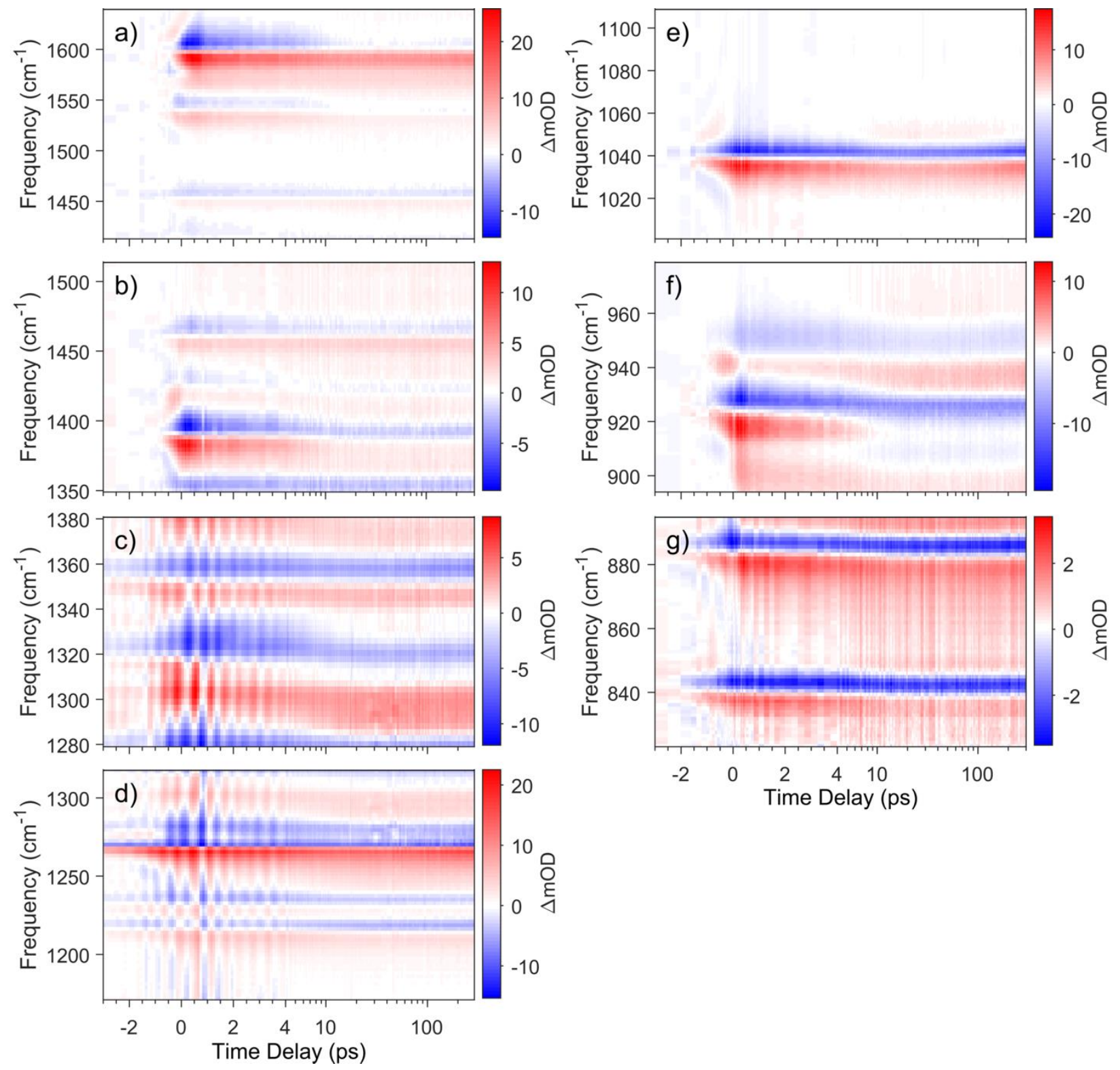

Figure 3. Transient spectra following excitation at $1260 \mathrm{~cm}^{-1}$ at each spectrometer grating positions. The oscillations near $\Delta t=0$ in c) and d) are the residual pump-probe interference pattern that was not fully removed by filtering; see text for additional details. Time-delays are linear up to 4 ps and logarithmic thereafter. See Fig. S11 for an alternate colormap. 

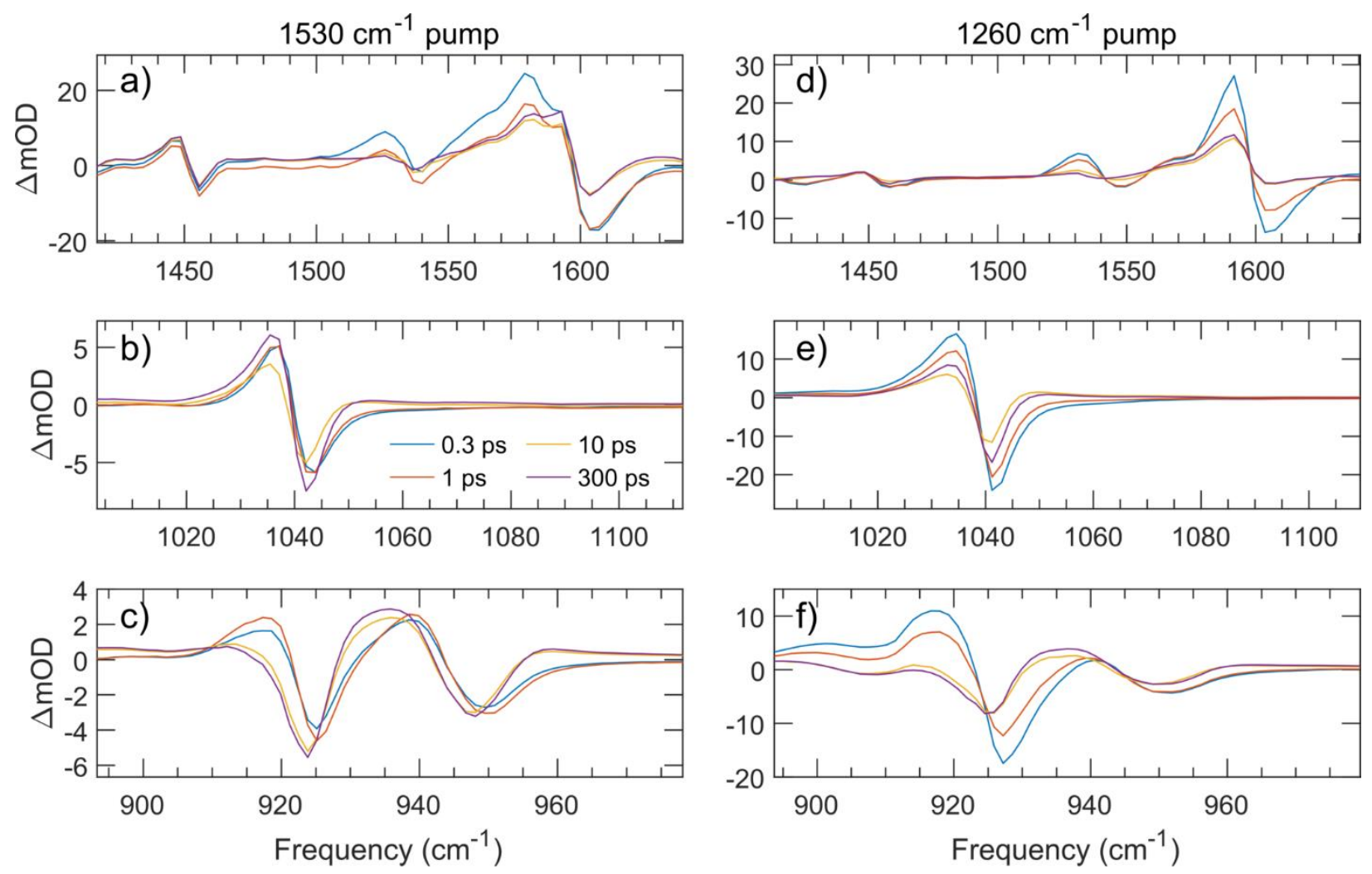

Figure 4. Example transient spectra at select time-delays following excitation at $1530 \mathrm{~cm}^{-1}(\mathrm{a}-\mathrm{c})$ and $1260 \mathrm{~cm}^{-1}(\mathrm{~d}-\mathrm{f})$. Spectra in a) are from the filtered results shown in Fig. 2 a). The full set of spectra may be found in Figures S10 and S12. 

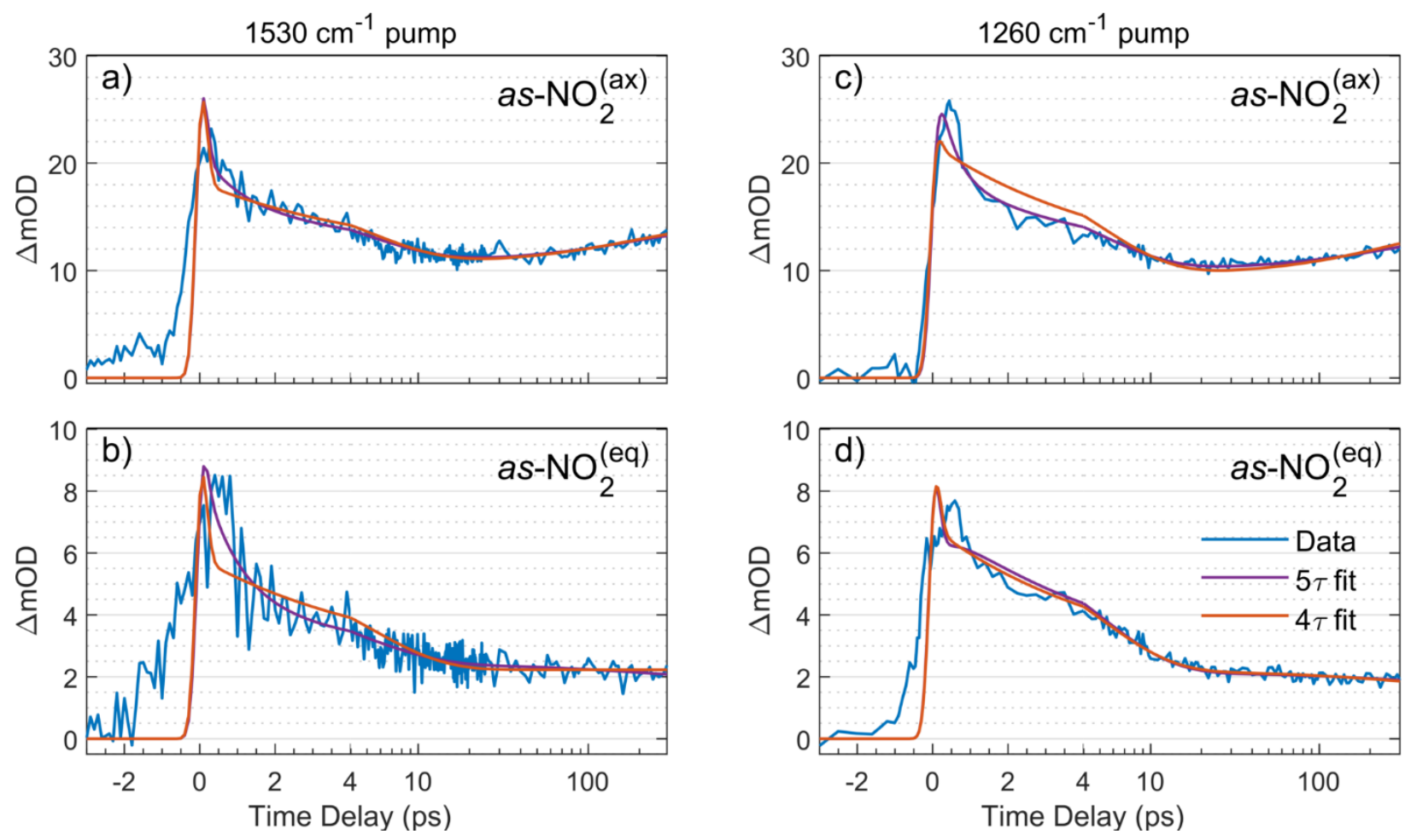

Figure 5. Transient behavior of the axial and equatorial asymmetric $\mathrm{NO}_{2}$ stretch modes following excitation at $1530 \mathrm{~cm}^{-1}$ ( $\mathrm{a}$ and b) and $1260 \mathrm{~cm}^{-1}$ (c and d). Example fits from two different kinetic models are also shown to highlight the contribution of a sub-ps time constant; see text for further details of the fit models. Frequencies shown are 1582, 1523, 1588, and $1531 \mathrm{~cm}^{-1}$ for parts a-d, respectively. Time-delays are linear up to $4 \mathrm{ps}$ and logarithmic thereafter. 


\section{DISCUSSION}

\section{A. Early Time Behavior}

We begin our discussion with the RDX dynamics associated with the initial excitation and sub0.3 ps appearance of transient signal. As with our previous report on VET dynamics in PETN, ${ }^{13}$ the prompt appearance of a broad-spectrum signal suggests an ultrafast VET step which couples all vibrational modes in RDX. Prompt VET was also reported by Shi et al. ${ }^{12}$ for RDX microcrystals, where 2D IR cross peaks were evident at dynamical times as early as $0.1 \mathrm{ps.} \mathrm{Such}$ rapid appearance of transient spectra, along with the reported 0.48 ps coherence dephasing time constant, ${ }^{12}$ strongly suggests that the initial excitation of modes across the spectrum is due to a coherent VET process. Though these initial dynamics occur within the time resolution of our experiment, there are pump-dependent spectral differences which suggest particular VET pathways. For example, in the experimental transient absorption spectra, the earliest features around $930 \mathrm{~cm}^{-1}$ show marked differences in shape and relative peak amplitudes between the two pump frequencies - compare Fig. 4 c) and f). Following photoexcitation with the $1530 \mathrm{~cm}^{-1}$ pump, decay of the excited $a s-\mathrm{NO}_{2}{ }^{(\mathrm{ax})}$ mode at $\sim 1590 \mathrm{~cm}^{-1}$ could excite the ring distortion, $\mathrm{CH}_{2} \mathrm{wag}$, and $\mathrm{NO}_{2}$ bending modes around $930 \mathrm{~cm}^{-1}$ and, by conservation of energy in a two-vibration scattering event, the in-plane ring breathing mode at $\sim 670 \mathrm{~cm}^{-1}$ — this latter mode was previously identified as a key VET intermediate in RDX. ${ }^{35}$ Similarly, the large initial transient response around 900$920 \mathrm{~cm}^{-1}$ from the $1260 \mathrm{~cm}^{-1}$ pump could be facilitated by coupling to the mode at $350 \mathrm{~cm}^{-1}$ (Refs. $35,47)$.

Broad-spectrum VET is supported by the dense $\triangle 2 \mathrm{PhDoS}$ shown in Fig. S2, which reveal multiple possible pathways for pairs of vibrational modes to couple. The clearest example of modeselectivity in the $\Delta 2 \mathrm{PhDoS}$ are the pumped (static Bose-Einstein population adjusted) modes at 
frequencies $<1000 \mathrm{~cm}^{-1}$, which show very few available scattering pathways to higher lying vibrations while the inverse process shows a plethora of available scattering events. To evaluate the most probable VET pathways, we would normally turn to the DFT predicted single mode relaxation times (SMRTs) of the RDX crystal. These SMRTs are the product of the $\triangle 2 \mathrm{PhDoS}$ and the scattering cross-section for each collision; ${ }^{62}$ however, our predicted SMRTs are unrealistically low, ranging from $0.01-0.1 \mathrm{ps}^{36}$ As the $\Delta 2 \mathrm{PhDoS}$ are strictly a count of allowable transitions, ${ }^{13}$ we are led to conclude that two-phonon collision cross-sections are the culprit for this poor prediction. While previous studies on metals and metal-oxides ${ }^{81,82}$ using the same computational method yield experimentally agreeable $1-10 \mathrm{ps} \mathrm{SMRTs,} \mathrm{these} \mathrm{materials} \mathrm{are} \mathrm{starkly} \mathrm{different} \mathrm{from}$ the molecular crystal studied here. Thus, without reliable two-phonon cross-sections, we cannot rigorously evaluate the most probable VET pathways and we limit our discussion to the above speculation. Further experimental work, such as a broadband 2D IR study (e.g. Ref. ${ }^{83}$ ), would provide considerable insight into these first VET steps.

After the prompt appearance of transient signal, multiple vibrational modes show evolution with sub-ps and 5 ps time constants from global fitting. The sub-ps time constant is similar to previously reported time constants in RDX microcrystals ${ }^{12}$ as well as the lifetimes extracted from temperature-dependent Raman linewidths of $\beta$-octahydro-1,3,5,7-tetranitro-1,3,5,7-tetrazocine crystals (HMX, a related EM) ${ }^{36}$ While it is tempting to separate and assign the two extracted time constants based solely on previous reports, we first consider our choice of kinetic model. For RDX microcrystals, Shi et al. ${ }^{12}$ report mode-specific time constants of $\sim 2$ and $4.11 \mathrm{ps}$ from simple exponential decay fits to single-frequency transients at the axial and equatorial as $-\mathrm{NO}_{2}$ stretching modes. To directly compare our present results with the fits from Shi et al., Fig. S22 shows lane integrated transients of the same spectral features following the $1530 \mathrm{~cm}^{-1}$ pump along with mono- 
exponential decay function fits. While our mono-exponential fits return similar time constants of $\sim 2$ and 5 ps, our trends do not match the previously reported results; ${ }^{12}$ the cause of this discrepancy remains unclear as, apart from a $\sim 60 \mathrm{~cm}^{-1}$ difference in central pump frequency and the use of microcrystals versus a thin film, the experiments appear to be comparable. Though the shorter, $\sim 2$ ps time constants could arise from simultaneous sub-ps and 5 ps decay dynamics, the extracted time-dependence of these features clearly depends on the kinetic model. While our assumption of a global sequential kinetic model is a useful description of correlated spectral changes across the probe window, it will not describe the multiple, parallel, and mode-specific VET pathways (and time constants) likely occurring following photoexcitation. Conversely, the independent fits to single frequencies by Shi et al. ${ }^{12}$ will extract mode-specific time constants but may not accurately describe the time dependence of broadening or spectral shifts.

While neither kinetic model is entirely correct, we can still draw a few conclusions by comparing different models to the experimental data. For example, the spectral regions requiring a sub-ps time constant — see the $4 \tau$ model description in the previous section—may be interpreted as effectively modifying the $5 \mathrm{ps}$ time constant to describe dynamics similar to those reported by Shi et al. ${ }^{12}$ (i.e. mode-selective, mono-exponential decays). Alternatively, the noticeably flat or slightly rising transient behavior around 1040 and $920 \mathrm{~cm}^{-1}$ following the $1530 \mathrm{~cm}^{-1}$ pump — shown in Fig. S19-strongly indicates competing decay and growth dynamics (i.e. two separate time constants). Indeed, rising signal at $\sim 920 \mathrm{~cm}^{-1}$ could support our proposed VET pathway coupling the initially excited as $-\mathrm{NO}_{2}{ }^{(\mathrm{ax})}$ mode at $\sim 1590 \mathrm{~cm}^{-1}$ to the modes at 930 and $670 \mathrm{~cm}^{-1}$. However, as with the initial, sub- 0.3 ps excitation of modes across the RDX spectrum, we are unable to firmly assign such pathways with the present results and again highlight the need for further experimental and computational work to decouple the numerous, competing VET pathways. 
The appearance of a $5 \mathrm{ps}$ time constant at both pump frequencies and for all the probed vibrational transitions strongly indicates that this time constant describes an aggregate VET behavior in RDX, though global fitting may miss mode-specific variations. Nonetheless, this 5 ps time constant corresponds to the clear evolution of all transient spectra to resemble the ThDIR spectrum shown in Fig. S14, with the caveat that direct quantitative comparisons between the transient spectra and ThDIR are inappropriate. As each VET process must necessarily couple to the low frequency vibrations to promote transitions, including the bath of long-range lattice motions, the strong similarity between the ThDIR and transient spectra for $\Delta t \geq 10 \mathrm{ps}$ suggests that the $5 \mathrm{ps}$ time constant is the time required for the vibrationally excited, high frequency modes to decay and repopulate their ground vibrational state, as supported by the pump-probe style MD simulations (Figures S3 and S4; see discussion below for details). However, vibrational relaxation of the high frequency modes does not result in the repopulation of the pre-excitation ground state. Instead, a shifted ground state is populated since the VET-excited low frequency lattice modes will perturb the higher frequency vibrations, ${ }^{6}$ manifesting as shifting and broadening of the line shapes (see Fig. S14 and Refs. ${ }^{13,36}$ for examples) and resulting in the reported transient spectra for $\Delta t \geq$ $10 \mathrm{ps}$. Thus, because of these clear spectral changes, we may firmly assign the 5 ps time constant to de-excitation of the high frequency vibrational modes in RDX.

\section{B. Long-Time Behavior}

\section{i. Signal Origin}

The final, $200 \mathrm{ps}$ time constant demonstrates that VET remains incomplete by $\Delta t=300 \mathrm{ps}$ in $\alpha$-RDX. Such long lived dynamics are not captured by standard theoretical treatments of VET ${ }^{84,85}$ and, to the best of our knowledge, have rarely been reported. ${ }^{13,14,86}$ In our previous investigation of VET in PETN, ${ }^{13}$ we speculated that a similar, $\sigma(100 \mathrm{ps})$ time constant was due to evolution of 
such an excited phonon distribution outside of the probe spectral window, though this conclusion remained tentative due to broad, overlapping spectral features. With the above assignment of the 5 ps time constant in RDX, the further 200 ps spectral evolution must be due to dynamics within the low frequency vibrations and lattice modes.

In RDX, the clear mode-selectivity of the 200 ps time constant thus suggests that select high frequency vibrations are more sensitive than previously thought to changes in the excited phonon bath. This enhanced sensitivity may imply strong coupling between low and high frequency vibrations, supporting a direct energy transfer ${ }^{4,27}$ model following a shockwave rather than a “doorway mode"-mediated multiphonon up-pumping scheme. ${ }^{1-3,87,88}$ To test that specific high frequency reporter vibrational modes are sensitive to long-lived phonon VET, we have recalculated the $\alpha$-RDX IR spectrum (see Fig. 1) with a 10\% static lattice compression to represent some characteristic of the experimental excited phonon bath. Figure 6 shows the ambient and compressed spectra for compression along the [010] crystal axis; similar compressions along the [100] and [001] axes each show unique peak shifting based on orientation of compression. Thus, these IR spectra of compressed RDX $i$ ) demonstrate that high frequency, "intramolecular" modes will be sensitive to lattice excitation (i.e. a breakdown of the rigid molecule approximation) and ii) suggest that the observed vibrational mode-selectivity arises from particular phonon excitations. However, without reliable two-phonon scattering cross-sections (see section 4.A) we cannot conclusively determine whether these reporter modes — which could potentially bypass a slow uppumping process - are sensitive to VET in select phonon modes or to broad-spectrum phonon evolution. 

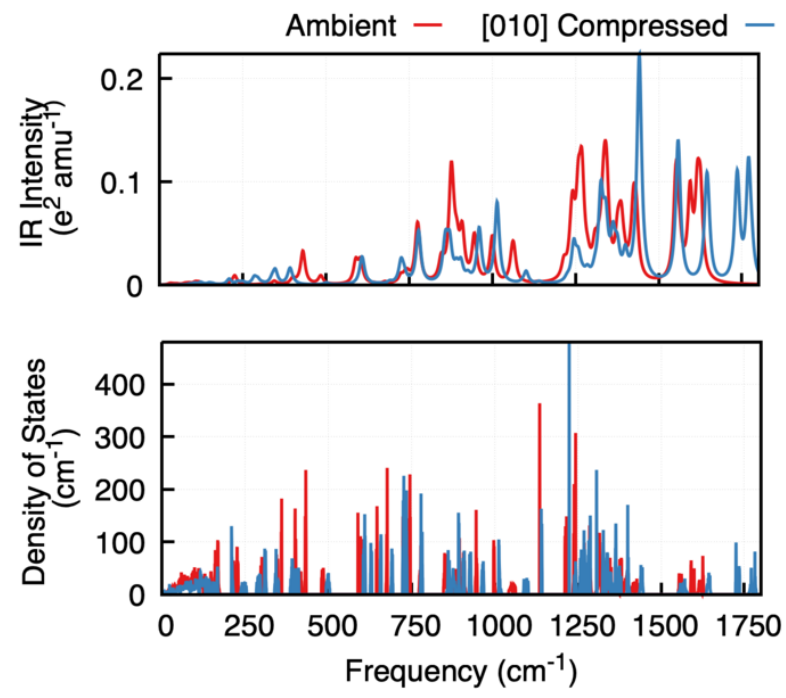

Figure 6. (Top) DFT calculated infrared spectrum of $\alpha$-RDX for both ambient and $10 \%$ compressed unit cells. Mild blue-shifting of all modes is observed, with as- $\mathrm{NO}_{2}$ being an exception where a large, $>50 \mathrm{~cm}^{-1}$, shift is present. (Bottom) Calculated total vibrational density of states again highlights inhomogeneous frequency shifts given the [010] lattice compression.

\section{ii. Low Frequency Vibrational Dynamics}

Similar to the case of PETN, ${ }^{13}$ we next consider two possible, phonon-centric explanations for the observed 200 ps dynamics. First, we could assume that the 5 ps time constant describes the rapid and complete thermal equilibration ${ }^{18}$ of pump-induced excess energy within the laser-sample interaction volume (i.e. that VET is complete by $\Delta t \approx 10 \mathrm{ps).} \mathrm{The} \mathrm{observed} \mathrm{long-time} \mathrm{spectral}$ changes would thus be due to spatial conduction of the locally higher temperature phonon distribution out of the laser-sample interaction volume. However, on the $300 \mathrm{ps}$ time scale of the present experiments, we would expect such cooling to only affect a small fraction of the total pumped volume. ${ }^{13,80}$ Furthermore, ThDIR spectra show decreasing signal intensity with decreasing temperature (Fig. S14 and Refs. ${ }^{13,80}$ ) in contrast with the observed increase in transient signal (see Figures 5 and S19-S21) on a 200 ps timescale. Thus, while thermal conduction of excited phonons out of the probe volume is certainly occurring, we believe this process alone does 
not explain the present observations. We are thus left with the second possible explanation for the longest time constant: that VET is incomplete by $\Delta t=300$ ps and we are observing continued evolution of a non-statistical, excited phonon distribution outside of the probe window-a conclusion again in marked contrast with previous theoretical treatments. ${ }^{18,89}$

To explore the dynamics of low frequency phonon modes, and avoid the requisite and prohibitive cost of DFT, we conducted MD simulations of mid-IR pumped vibrations looking for signatures of long-lived, non-equilibrium dynamics. Figure 7 shows the MD predicted phonon mode SMRTs comparing ambient density and compressed $\alpha$-RDX, where the vibrations at 1266 $\mathrm{cm}^{-1}$ (ambient pressure) and $1280 \mathrm{~cm}^{-1}(10 \%$ compression along the [010] axis) have been pumped. Additionally, a total and partial vibrational density of states is plotted that highlights axial or equatorial $\mathrm{NO}_{2}$ contribution. Similar to Figures 2 and 3, the bottom panels of Fig. 7 show the evolving total vibrational density of states through time, where the color scale indicates if modes are in higher (red) or lower (blue) population relative to equipartition of energy. Importantly, these low frequency phonons are initially cold relative to the pumped vibrations, meaning that — while the pump is present- these vibrations act as a sink for the energy deposited into the system. In contrast with the DFT-derived SMRTs, here the SMRTs are derived from frequency-dependent, mono-exponential fits to the evolving mode populations; a single SMRT thus captures the most prominent dynamics occurring after the pump thermostat is removed. MD spectra in other probe frequency ranges, as well as additional simulations for different pumped modes, are available in section S2.3 of the SI.

The most striking result is a marked increase in the low frequency SMRTs when the RDX crystal is compressed, where again this compression is used to mimic some character of an excited phonon bath. In particular, phonons involving $\mathrm{NO}_{2}$ motion (namely 180,260 , and $305 \mathrm{~cm}^{-1}$ ) show 
significant blue-shifting and broadening due to the compression. The longest lived of these excitations show SMRTs of 40-50ps, which are in stark contrast to the compression-insensitive SMRT of 1-5 ps near the pumped mode, as shown in Fig. S3. These elongated SMRTs cannot be attributed to irreversible peak shifts and broadening as both ambient and compressed samples were elevated by the pumped mode to very similar final temperatures ( 357 and $361 \mathrm{~K}$, respectively). Though the initial, compression-insensitive VET dynamics of high frequency modes are somewhat surprising given large peak shifts shown in Fig. 6, a constant high frequency SMRT further supports the separation of timescales based on VET mechanism seen in experiments. Furthermore, the largest reliable SMRT of the compressed sample ( $\sim 50 \mathrm{ps})$ challenges the assumption made that the spectra at $100 \mathrm{ps}$ can be taken as fully thermalized. While the compressed SMRTs do not yet match the experimental time constant of $200 \mathrm{ps}$, the SMRTs assume independent decay dynamics for each mode and the difference in extracted time constants suggests some degree of sequential, phonon-centered VET dynamics. Thus, these MD results suggest that an excited phonon distribution produced by the initial VET steps will itself result in elongated relaxation dynamics for lower frequency vibrations-a key, novel insight into the energy redistribution mechanisms and timescales of EMs. Follow on MD work will need to focus on the relationship between modespecific VET dynamics following a mechanical shock and the initial chemical reactions in RDX. 

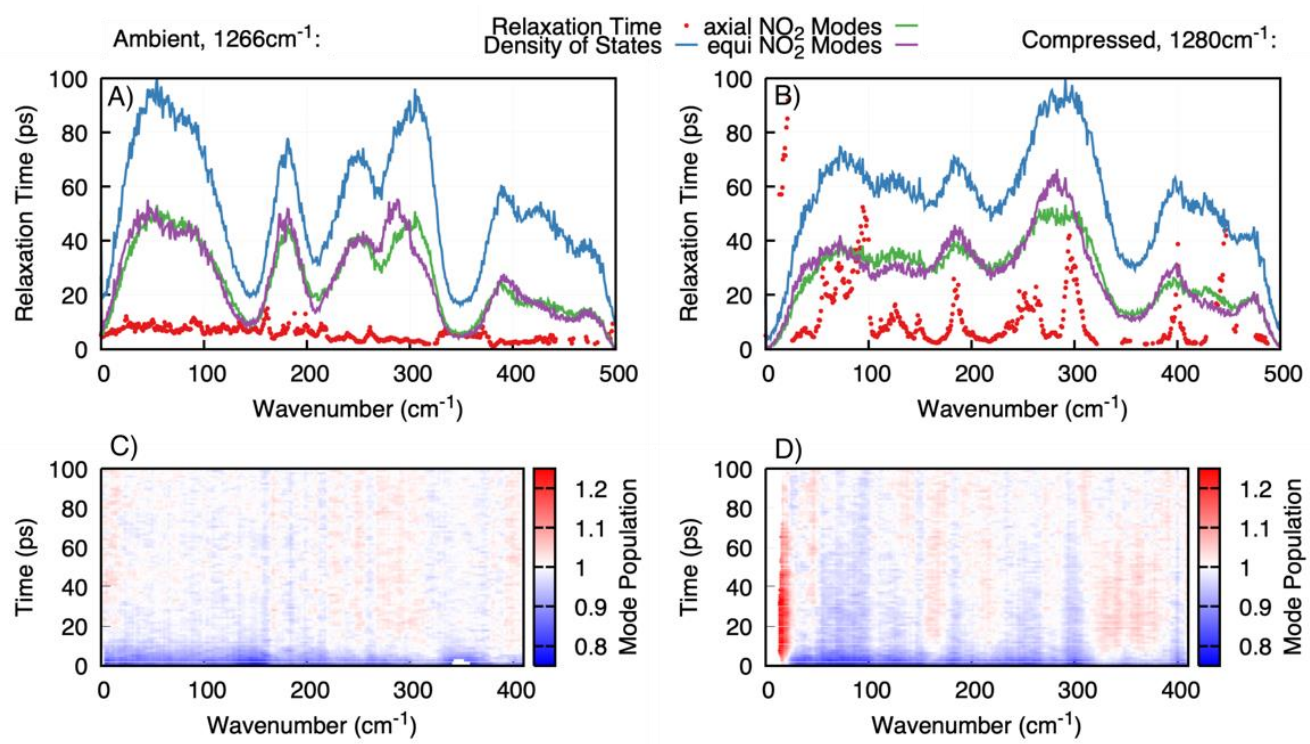

Figure 7. Pumped vibration is the $\mathrm{N}-\mathrm{N}^{(\mathrm{eq})}$ stretch, $\mathrm{CH}_{2}$ bend, and $s s-\mathrm{NO}_{2}$ mode $\left(1260 \mathrm{~cm}^{-1}\right.$, ambient pressure) in both sets of data, time origin is where the pump is removed from the probe volume. (a) Extracted exponential decay time constants as the uncompressed RDX crystal returns to an equipartition of energy among all vibrations. Total density of states (blue) and partial density of states (green, purple) highlight modes with axial versus equatorial $\mathrm{NO}_{2}$ character. (b) Time constants per-mode for a compressed sample of RDX. Two-dimensional density of states normalized to an equipartition of energy (labeled as Mode Population) for (c) ambient and (d) compressed RDX; blue regions are under-populated and red over-populated with respect to the spectra at the same temperature. See Figures S3-S5 for additional MD results.

\section{iii. Mode-Selectivity}

With a firm grasp of the origin and nature of the long-time dynamics in RDX, we finally turn our attention to the observed mode-selectivity. As an $\sigma(100 \mathrm{ps})$ time constant has now been observed in multiple EM thin films, ${ }^{13,14}$ the separation of VET timescales seems to be a materialindependent feature and correlating the longest dynamics with likewise material-independent properties would be of significant interest. Since the specific vibrational modes are inherently material-dependent, we would like to understand the mode-selective VET through the chemically intuitive lenses of select bond excitation or of other chemical moieties. In our previous study of 
PETN,${ }^{13}$ the $\sigma(100 \mathrm{ps})$ evolution was tentatively associated with modes involving the reactive nitrate ester stretch $\left(\mathrm{RO}-\mathrm{NO}_{2}\right)$ coordinate. Though a direct correspondence between vibrational mode and chemical moiety is only an accurate description in the gas-phase, such an analysis has previously suggested facile energy partitioning into the $\mathrm{N}-\mathrm{N}$ stretch of $\mathrm{RDX} \cdot{ }^{27}$ With the above caveat in mind, we thus seek such a relationship between the longest, mode-selective dynamics and an intuitive chemical bond model.

From the list of the spectral regions which show a 200 ps evolution-see section 3.B-a possible unifying characteristic appears to be vibrational modes with $\mathrm{N}-\mathrm{N}$ stretch character; for example, the mode associated with the strong time-dependent change at $1040 \mathrm{~cm}^{-1}$ appears to include significant $\mathrm{N}-\mathrm{N}^{(\mathrm{eq})}$ stretch character (see Fig. 1 f) and Table S1). However, this initial assessment is based on qualitative descriptions of mode character from the vector displacement diagrams of only one RDX molecule in the unit cell. To assess potential trends more rigorously, we have decomposed the DFT calculated IR spectrum along several likely dimensions. For all molecules within the $\alpha$-RDX unit cell, each mass-weighted harmonic mode is characterized by: $i$ ) fractional displacement of each atom type, $i i$ ) projection onto the unit cell axes (as suggested by Fig. 6), iii) fractional change of each $\mathrm{N}-\mathrm{N}$ bond length (from our initial assessment and Refs. ${ }^{27,29}$ ), and $i v$ ) fractional change of each $\mathrm{N}-\mathrm{O}$ bond length (from Fig. $7 \mathrm{~b}$ ) and the observed dynamics around $\left.1590 \mathrm{~cm}^{-1}\right)$. Of the 504 degrees of freedom in uncompressed $\alpha-\mathrm{RDX}, 61$ vibrations have a calculated IR intensity $\geq 0.1 \mathrm{e}^{2} \mathrm{amu}^{-1} ; 44$ of these modes are within the experimental probe window. Based on the overlap of the calculated and experimental IR spectra in Fig. 1, and without considering our assigned mode character, each of these 44 modes was then assigned a "yes/no" value depending on whether the calculated frequency $(\mathrm{CF})$ matches an experimental mode evolves with the 200 ps time constant. A third "neutral" value was assigned to modes whose CF could not 
be unambiguously mapped to experimental frequencies; the resulting annotated list of 61 frequencies is given in Table S2. Two examples of these decomposed IR spectra, averaged over all molecules within the unit cell, are shown in Fig. 8 with the full set shown in Figures S6 and S7; red peaks highlight modes which evolve with the 200 ps time constant while peak intensity scales with fractional change in atomic displacement, projection onto a crystal axis, or relative $\mathrm{N}-\mathrm{X}$ bond length.

In general, spectra reporting either atomic fractions of each mode or the projections onto the crystal axis (Fig. S6) do not show any obvious, global correlation with the 200 ps temporal behavior, though large $\mathrm{N}$ atom and $\mathrm{O}$ atom motions are present in the $a s-\mathrm{NO}_{2}{ }^{(\mathrm{ax})}$ modes at $\mathrm{CF} \approx$ $1610 \mathrm{~cm}^{-1}$. It is important to note that while compression along the principal crystal axes implied phonon-induced, mode-selective shifts to the high frequency vibrational spectra in general—see section 4.B.i- these particular projections do not correlate to the experimentally observed $200 \mathrm{ps}$ behavior. In contrast, spectra detailing the average relative displacements of the $\mathrm{N}-\mathrm{N}$ and $\mathrm{N}-\mathrm{O}$ bonds, shown in Figures 8 and S7, begin to show some correspondence with the longest dynamics. For example, modes with the largest relative change in $\mathrm{N}-\mathrm{N}^{(\mathrm{eq})}$ bond length tend to evolve with the 200 ps time constant while, excepting the as $-\mathrm{NO}_{2}{ }^{(\mathrm{ax})}$ modes, larger changes to the $\mathrm{N}-\mathrm{O}^{(\mathrm{ax})}$ bonds appear to favor modes which do not evolve with the 200 ps time constant. Indeed, the correlation to $\mathrm{N}-\mathrm{N}$ bond excitation appears strongest when plotting the maximum change in $\mathrm{N}-\mathrm{N}$ bond length across all three $\mathrm{N}-\mathrm{N}$ bonds, as shown in Fig. S7. Pairwise correlation plots comparing these spectral decompositions, as shown in Figures 8 c) and S8, reinforce the above conclusions: atomic displacement or crystal alignment typically results in no obvious pattern while displacements of the $\mathrm{N}-\mathrm{N}$ vs. $\mathrm{N}-\mathrm{O}$ bonds generally correlate evolution with the 200 ps time constant to excitation of the $\mathrm{N}-\mathrm{O}^{(\mathrm{ax})}$ or $\mathrm{N}-\mathrm{N}^{(\mathrm{eq})}$ bonds. 
While these spectral decompositions generally support a connection between excitation of the $\mathrm{N}-\mathrm{N}$ bond and long-time spectral evolution, even the strongest correlations - such as plotted in Fig. 8 c) - reveal multiple modes with minimal $\mathrm{N}-\mathrm{O}^{(\mathrm{ax})}$ and $\mathrm{N}-\mathrm{N}^{(\mathrm{eq})}$ bond excitation which nonetheless evolve with the $200 \mathrm{ps}$ time constant and are as of yet unexplained. Given that the 200 ps time constant arises from long-range lattice motions, this bond-centric, intramolecular picture is undoubtedly insufficient and we would need to consider changes to the larger system (i.e. extended intermolecular interactions) to determine the underlying connection. As the approximation of using a single unit cell when employing the FDM is already evident for low frequencies within the probe window—see section 3.A—accurate correlations between intra- and intermolecular modes requires further computational effort beyond the scope of the present work. Nonetheless, these initial efforts reinforce the importance ${ }^{27}$ of the reactive $\mathrm{N}-\mathrm{N}$ bond in understanding the initial energy transfer dynamics in RDX. 

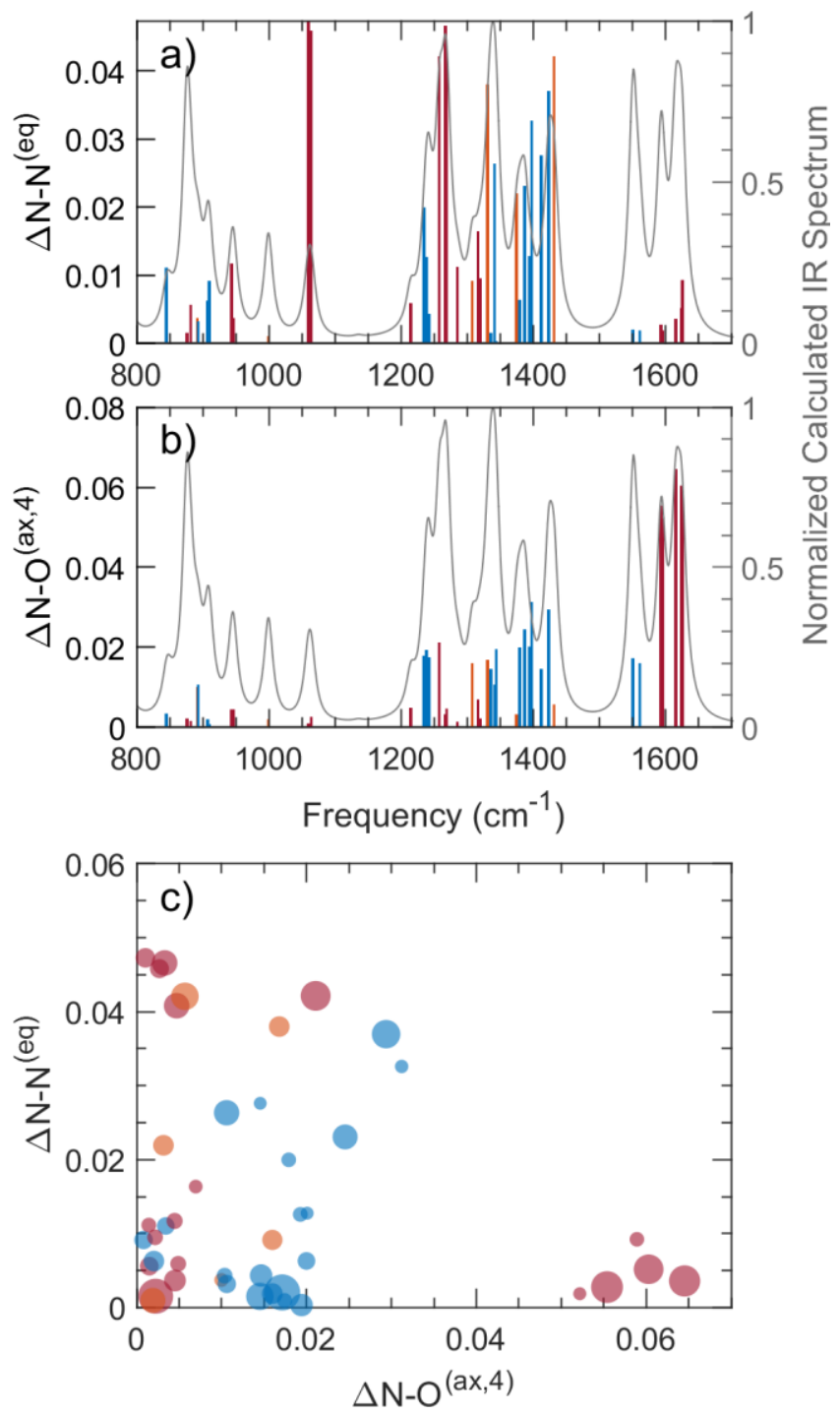

Figure 8. Example decomposed spectra showing the fractional bond length change from equilibrium in (a) the $\mathrm{N}-\mathrm{N}^{(e q)}$ or (b) one of the four $\mathrm{N}-\mathrm{O}^{(a x)}$ bonds for each frequency. Bar height reports fraction while bar color highlights frequencies which experimentally demonstrate $200 \mathrm{ps}$ evolution: red = "yes," blue = "no," and black = "neutral" (i.e. no unambiguous mapping to experiment). c) Example correlation plot comparing the relative fractional change in an $\mathrm{N}-\mathrm{O}^{(\mathrm{ax})}$ versus the $\mathrm{N}-\mathrm{N}^{(\mathrm{eq})}$ bond length for each frequency, highlighting large $\mathrm{N}-\mathrm{N}^{(\mathrm{eq})}$ and small $\mathrm{N}-\mathrm{O}^{(\mathrm{ax})}$ displacements as a reasonable predictor of long-time spectral evolution. Marker size scales with calculated IR intensity while marker color highlights frequencies which experimentally demonstrate $200 \mathrm{ps}$ evolution. Additional decomposed spectra and correlation plots may be found in Figures S6-S8.

\section{CONCLUSIONS}


In the present work, we have employed a combination of experiment and theory to demonstrate incomplete VET in RDX on hundreds of picosecond timescales, challenging commonly utilized theories of shock initiation in these molecular crystals. If we assume that the VET timescales and pathways are reversible (phonon up-pumping versus vibrational relaxation), then the results herein of $\sim 200$ ps, phonon-mediated VET, point away from the "doorway mode" concept for shock initiation ${ }^{1,2}$ in favor of direct coupling to high frequency reporter modes ${ }^{4,27}$ which lie along commonly recognized reaction pathways. ${ }^{90,91}$ Indeed, our observations of $\sigma(100$ ps) dynamics in the three energetic materials we have presently or previously investigated—RDX, PETN, ${ }^{13}$ and $\mathrm{TATB}^{14}$ - suggest that the direct coupling of phonons to reactive high frequency modes, and the breakdown of the rigid molecule approximation, may be a general feature of energetic molecular crystals. The observed mode-selectivity in these longest dynamics, coupled with DFT simulations showing compression orientation-dependent peak shifts, may provide key clues to the observed crystallographic orientation dependence on shock sensitivity. ${ }^{92}$ Finally, these MD results under static compression suggest that, following shock compression, excited phonon relaxation times will be substantially longer than have been previously assumed. ${ }^{18}$ Such a persistent, non-equilibrium phonon distribution would have more opportunity to excite higher frequency, chemically reactive coordinates through either the multiphonon up-pumping ${ }^{1,2}$ or direct coupling $^{4,27}$ pathways. Further experimental work, such as transient infrared spectroscopy of a molecular crystal under static compression, would validate these calculations and add significant insights into the interplay between compression, phonon lifetime, and VET mechanisms.

Two outstanding experimental questions remain from our present work. First, what are the ultrafast pathways responsible for the sub-0.3 ps dynamics? Elucidating these first VET steps would help explain the pump-dependent differences in the earliest transient spectra—cf. Figures 4 
c) and f). Second, what are the underlying chemical characteristics responsible for the modeselective 200 ps dynamics? In both cases, broadband 2D IR studies would unveil key mechanistic pathways critical to understanding the structure-dynamics-function relationships of EM bulk properties. Further work utilizing IR pump-THz probe holds promise to directly test the timescales of VET within the low frequency phonon modes. Finally, as our samples are well-controlled thin films of material, more work is also needed to understand the phase, morphology, and temperature dependence of VET as well as whether the phonon-mediated, reporter modes are preserved. Extending the above suite of experiments to include static pressure loads would enable new EM polymorphs to be studied as well as explore the relationship between pressure and extended phonon lifetimes. VET studies of bulk polycrystalline EM samples could further contribute to understanding the interplay between the longest dynamics observed herein and the role of microstructure (e.g. grain boundaries ${ }^{93,94}$ ) and hot-spot formation ${ }^{1,2,95,96}$ in the shock-to-detonation transition.

Though further work is needed to develop a fundamental understanding of shock sensitivity from VET timescales and pathways, such an explicit connection between VET and initial chemical reactivity would mark a critical step forward in our understanding of shock sensitivity. Indeed, currently used destructive methods for characterizing sensitivity—such as drop weight ${ }^{97}$ or friction testing ${ }^{98}$ — could be augmented or replaced with non-destructive characterizations of VET. Recent work by McGrane and co-workers ${ }^{9,10}$ measuring the ultrafast behavior of shocked, thin film samples with broadband mid-IR sources highlights the converging applicability of these spectroscopic techniques. Such augmentation would be advantageous as both a safer way to screen newly synthesized compounds as well as a means to diagnose the state-of-health of energetic materials already in use. 
Supporting Information. Characterization of the RDX thin film samples, additional theoretical results, additional experimental results, and details of the Zenodo data archive.

Author Information. The authors declare no competing financial interests.

Acknowledgements. The authors thank Michael Marquez for depositing the RDX samples and Deneille Wiese-Smith for handling storage and transport of RDX. The authors also thank M. Kathleen Alam and Darcie Farrow for their careful reading and technical feedback on this manuscript. This work is supported by the Laboratory Directed Research and Development program at Sandia National Laboratories, a multi-mission laboratory managed and operated by National Technology and Engineering Solutions of Sandia, LLC., a wholly owned subsidiary of Honeywell International, Inc., for the U.S. Department of Energy's National Nuclear Security Administration under contract DE-NA-0003525. This paper describes objective technical results and analysis. Any subjective views or opinions that might be expressed in the paper do not necessarily represent the views of the U.S. Department of Energy or the United States Government.

Data Accessibility. Experimental and computational data underpinning the present manuscript are freely available through the Zenodo data archive at DOI:10.5281/zenodo.4663415.

\section{References.}

(1) Dlott, D. D.; Fayer, M. D. Shocked Molecular Solids: Vibrational up Pumping, Defect Hot Spot Formation, and the Onset of Chemistry. J. Chem. Phys. 1990, 92 (6), 3798-3812. https://doi.org/10.1063/1.457838.

(2) Tokmakoff, A.; Fayer, M. D.; Dlott, D. D. Chemical Reaction Initiation and Hot-Spot Formation in Shocked Energetic Molecular Materials. J. Phys. Chem. 1993, 97 (9), 19011913. https://doi.org/10.1021/j100111a031. 
(3) Michalchuk, A. A. L.; Hemingway, J.; Morrison, C. A. Predicting the Impact Sensitivities of Energetic Materials through Zone-Center Phonon up-Pumping. J. Chem. Phys. 2021, 154 (6), 064105. https://doi.org/10.1063/5.0036927.

(4) Coffey, C. S.; Toton, E. T. A Microscopic Theory of Compressive Wave-induced Reactions in Solid Explosives. J. Chem. Phys. 1982, 76 (2), 949-954. https://doi.org/10.1063/1.443065.

(5) Dlott, D. D. Optical Phonon Dynamics in Molecular Crystals. Annu. Rev. Phys. Chem. 1986, 37 (1), 157-187. https://doi.org/10.1146/annurev.pc.37.100186.001105.

(6) Califano, S.; Schettino, V. Vibrational Relaxation in Molecular Crystals; 1988; Vol. 7. https://doi.org/10.1080/01442358809353204.

(7) Veysset, D.; Pezeril, T.; Kooi, S.; Bulou, A.; Nelson, K. A. Laser-Induced versus Shock Wave Induced Transformation of Highly Ordered Pyrolytic Graphite. Appl. Phys. Lett. 2015, 106 (16), 161902. https://doi.org/10.1063/1.4918929.

(8) Dresselhaus-Cooper, L. E.; Martynowych, D. J.; Zhang, F.; Tsay, C.; Ilavsky, J.; Wang, S. G.; Chen, Y.-S.; Nelson, K. A. Pressure-Thresholded Response in Cylindrically Shocked Cyclotrimethylene Trinitramine (RDX). J. Phys. Chem. A 2020, 124 (17), 3301-3313. https://doi.org/10.1021/acs.jpca.9b07637.

(9) Powell, M. S.; Sakano, M. N.; Cawkwell, M. J.; Bowlan, P. R.; Brown, K. E.; Bolme, C. A.; Moore, D. S.; Son, S. F.; Strachan, A.; McGrane, S. D. Insight into the Chemistry of PETN Under Shock Compression Through Ultrafast Broadband Mid-Infrared Absorption Spectroscopy. J. Phys. Chem. A 2020, 124 (35), 7031-7046. https://doi.org/10.1021/acs.jpca.0c03917. 
(10) Powell, M. S.; Moore, D. S.; McGrane, S. D. Insight into the Chemistry of TNT during Shock Compression through Ultrafast Absorption Spectroscopies. J. Chem. Phys. 2021, 154 (5), 054201. https://doi.org/10.1063/5.0032018.

(11) Ostrander, J. S.; Knepper, R.; Tappan, A. S.; Kay, J. J.; Zanni, M. T.; Farrow, D. A. Energy Transfer between Coherently Delocalized States in Thin Films of the Explosive Pentaerythritol Tetranitrate (PETN) Revealed by Two-Dimensional Infrared Spectroscopy. J. Phys. Chem. B 2017, 121 (6), 1352-1361. https://doi.org/10.1021/acs.jpcb.6b09879.

(12) Shi, L.; Yu, P.; Zhao, J.; Wang, J. Ultrafast Intermolecular Vibrational Energy Transfer in Hexahydro-1,3,5-Trinitro-1,3,5-Triazine in Molecular Crystal by 2D IR Spectroscopy. J. Phys. Chem. C 2020, 124 (4), 2388-2398. https://doi.org/10.1021/acs.jpcc.9b11030.

(13) Cole-Filipiak, N. C.; Knepper, R.; Wood, M.; Ramasesha, K. Sub-Picosecond to SubNanosecond Vibrational Energy Transfer Dynamics in Pentaerythritol Tetranitrate. J. Phys. Chem. Lett. 2020, 6664-6669. https://doi.org/10.1021/acs.jpclett.0c01780.

(14) Ramasesha, K.; Wood, M.; Cole-Filipiak, N.; Knepper, R. Experimental and Theoretical Studies of Ultrafast Vibrational Energy Transfer Dynamics in Energetic Materials.; Albuquerque, NM, and Livermore, CA (United States), 2020. https://doi.org/10.2172/1671386.

(15) Hill, J. R.; Dlott, D. D. Theory of Vibrational Cooling in Molecular Crystals: Application to Crystalline Naphthalene. J. Chem. Phys. 1988, 89 (2), 842-858. https://doi.org/10.1063/1.455207.

(16) Chen, S.; Tolbert, W. A.; Dlott, D. D. Direct Measurement of Ultrafast Multiphonon Up- 
Pumping in High Explosives. J. Phys. Chem. 1994, 98 (32), 7759-7766.

https://doi.org/10.1021/j100083a004.

(17) Aubuchon, C. M.; Rector, K. D.; Holmes, W.; Fayer, M. D. Nitro Group Asymmetric Stretching Mode Lifetimes of Molecules Used in Energetic Materials. Chem. Phys. Lett. 1999, 299 (1), 84-90. https://doi.org/10.1016/S0009-2614(98)01241-X.

(18) Hooper, J. Vibrational Energy Transfer in Shocked Molecular Crystals. J. Chem. Phys. 2010, 132 (1), 014507. https://doi.org/10.1063/1.3273212.

(19) Choi, C. S.; Prince, E. The Crystal Structure of Cyclotrimethylenetrinitramine. Acta Crystallogr. Sect. B Struct. Crystallogr. Cryst. Chem. 1972, 28 (9), 2857-2862. https://doi.org/10.1107/S0567740872007046.

(20) Karpowicz, R. J.; Brill, T. B. Librational Motion of Hexahydro-1,3,5-Trinitro-s-Triazine Based on the Temperature Dependence of the Nitrogen-14 Nuclear Quadrupole Resonance Spectra: The Relationship to Condensed-Phase Thermal Decomposition. $J$. Phys. Chem. 1983, 87 (12), 2109-2112. https://doi.org/10.1021/j100235a017.

(21) Karpowicz, R. J.; Brill, T. B. Comparison of the Molecular Structure of Hexahydro-1,3,5Trinitro-s-Triazine in the Vapor, Solution, and Solid Phases. J. Phys. Chem. 1984, 88 (3), 348-352. https://doi.org/10.1021/j150647a005.

(22) Politzer, P.; Ma, Y. Noncovalent Intermolecular Energetics: RDX Crystal. Int. J. Quantum Chem. 2004, 100 (5), 733-739. https://doi.org/10.1002/qua.20237.

(23) Wight, C. A.; Botcher, T. R. Thermal Decomposition of Solid RDX Begins with N-N Bond Scission. J. Am. Chem. Soc. 1992, 114 (21), 8303-8304. 
https://doi.org/10.1021/ja00047a059.

(24) Kim, E.; Thynell, S. Condensed-Phase Kinetic Rates of RDX from Confined Rapid Thermolysis/FTIR Spectroscopy. In 34th AIAA/ASME/SAE/ASEE Joint Propulsion Conference and Exhibit; American Institute of Aeronautics and Astronautics: Reston, Virigina, 1998. https://doi.org/10.2514/6.1998-3828.

(25) Maharrey, S.; Behrens, R. Thermal Decomposition of Energetic Materials. 5. Reaction Processes of 1,3,5-Trinitrohexahydro-s-Triazine below Its Melting Point. J. Phys. Chem. A 2005, 109 (49), 11236-11249. https://doi.org/10.1021/jp054188q.

(26) Patterson, J. E.; Dreger, Z. A.; Miao, M.; Gupta, Y. M. Shock Wave Induced Decomposition of RDX: Time-Resolved Spectroscopy. J. Phys. Chem. A 2008, 112 (32), 7374-7382. https://doi.org/10.1021/jp800827b.

(27) Kraczek, B.; Chung, P. W. Investigation of Direct and Indirect Phonon-Mediated Bond Excitation in $\alpha$-RDX. J. Chem. Phys. 2013, 138 (7), 074505. https://doi.org/10.1063/1.4790637.

(28) Kumar, G.; Van Gessel, F. G.; Elton, D. C.; Chung, P. W. Phonon Lifetimes and Thermal Conductivity of the Molecular Crystal $\alpha$-RDX. MRS Adv. 2019, 4 (40), 2191-2199. https://doi.org/10.1557/adv.2019.278.

(29) Kumar, G.; VanGessel, F. G.; Munday, L. B.; Chung, P. W. 3-Phonon Scattering Pathways for Vibrational Energy Transfer in Crystalline RDX. arXiv.org 2021, 2104.11762.

(30) Dang, N. C.; Dreger, Z. A.; Gupta, Y. M.; Hooks, D. E. Time-Resolved Spectroscopic 
Measurements of Shock-Wave Induced Decomposition in Cyclotrimethylene Trinitramine (RDX) Crystals: Anisotropic Response. J. Phys. Chem. A 2010, 114 (43), 11560-11566. https://doi.org/10.1021/jp106892c.

(31) An, Q.; Liu, Y.; Zybin, S. V.; Kim, H.; Goddard, W. A. Anisotropic Shock Sensitivity of Cyclotrimethylene Trinitramine (RDX) from Compress-and-Shear Reactive Dynamics. $J$. Phys. Chem. C 2012, 116 (18), 10198-10206. https://doi.org/10.1021/jp300711m.

Bai, Z.-Q.; Dai, B.; Chang, J.; Wang, J.; Ge, N.-N. Theoretical Study for Anisotropic Responses of the Condensed-Phase RDX under Shock Loadings. J. Mol. Graph. Model. 2018, 85, 316-322. https://doi.org/10.1016/j.jmgm.2018.08.009.

(33) Dick, J. J. Anomalous Shock Initiation of Detonation in Pentaerythritol Tetranitrate Crystals. J. Appl. Phys. 1997, 81 (2), 601-612. https://doi.org/10.1063/1.364201.

(34) Mathew, N.; Picu, C. R.; Chung, P. W. Peierls Stress of Dislocations in Molecular Crystal Cyclotrimethylene Trinitramine. J. Phys. Chem. A 2013, 117 (25), 5326-5334. https://doi.org/10.1021/jp401368t.

(35) Yu, G.; Zeng, Y.; Guo, W.; Wu, H.; Zhu, G.; Zheng, Z.; Zheng, X.; Song, Y.; Yang, Y. Visualizing Intramolecular Vibrational Redistribution in Cyclotrimethylene Trinitramine (RDX) Crystals by Multiplex Coherent Anti-Stokes Raman Scattering. J. Phys. Chem. A 2017, 121 (13), 2565-2571. https://doi.org/10.1021/acs.jpca.7b00069.

(36) McGrane, S. D.; Barber, J.; Quenneville, J. Anharmonic Vibrational Properties of Explosives from Temperature-Dependent Raman. J. Phys. Chem. A 2005, 109 (44), 99199927. https://doi.org/10.1021/jp0523219. 
(37) Knepper, R.; Tappan, A. S.; Wixom, R. R.; Rodriguez, M. A. Controlling the Microstructure of Vapor-Deposited Pentaerythritol Tetranitrate Films. J. Mater. Res. 2011, 26 (13), 1605-1613. https://doi.org/10.1557/jmr.2011.177.

(38) Knepper, R.; Forrest, E. C.; Marquez, M. P.; Tappan, A. S. Effect of Microstructure on the Detonation Behavior of Vapor-Deposited Pentaerythritol Tetranitrate (PETN) Films; 2018; p 150022. https://doi.org/10.1063/1.5044978.

(39) Forrest, E. C.; Knepper, R.; Brumbach, M. T.; Rodriguez, M. A.; Archuleta, K.; Marquez, M. P.; Tappan, A. S. Engineering the Microstructure and Morphology of Explosive Films via Control of Interfacial Energy. ACS Appl. Mater. Interfaces 2021, 13 (1), 1670-1681. https://doi.org/10.1021/acsami.0c10193.

(40) Castle, J. E. The Composition of Metal Surfaces After Atmospheric Exposure: An Historical Perspective. J. Adhes. 2008, 84 (4), 368-388. https://doi.org/10.1080/00218460802004477.

(41) Bebelis, S.; Nikolopoulos, P. Temperature Dependence of the Surface Energy of the LowIndex Planes of CaF2, BaF2 and SrF2. J. Mater. Eng. Perform. 2017, 26 (3), 1223-1228. https://doi.org/10.1007/s11665-017-2560-7.

(42) Brady, J. J.; Argirakis, B. L.; Gordon, A. D.; Lareau, R. T.; Smith, B. T. Polymorphic Phase Control of RDX-Based Explosives. Appl. Spectrosc. 2018, 72 (1), 28-36. https://doi.org/10.1177/0003702817712259.

(43) Karpowicz, R. J.; Sergio, S. T.; Brill, T. B. $\beta$-Polymorph of Hexahydro-1,3,5-Trinitro-sTriazine. A Fourier Transform Infrared Spectroscopy Study of an Energetic Material. Ind. Eng. Chem. Prod. Res. Dev. 1983, 22 (2), 363-365. https://doi.org/10.1021/i300010a038. 
(44) Cole-Filipiak, N. C.; Marquez, M.; Knepper, R.; Harmon, R.; Wiese-Smith, D.; Schrader, P.; Wood, M.; Ramasesha, K. Ultrafast Spectroscopic Studies of Vibrational Energy Transfer in Energetic Materials. AIP Conf. Proc. 2020, 2272 (November). https://doi.org/10.1063/12.0000848.

(45) Petersen, P. B.; Tokmakoff, A. Source for Ultrafast Continuum Infrared and Terahertz Radiation. Opt. Lett. 2010, 35 (12), 1962. https://doi.org/10.1364/OL.35.001962.

(46) Fayer, M. D. Ultrafast Infrared And Raman Spectroscopy; Practical spectroscopy; CRC Press, 2001.

(47) Infante-Castillo, R.; Pacheco-Londoño, L.; Hernández-Rivera, S. P. Vibrational Spectra and Structure of RDX and Its ${ }^{13} \mathrm{C}$ - and ${ }^{15} \mathrm{~N}$-Labeled Derivatives: A Theoretical and Experimental Study. Spectrochim. Acta - Part A Mol. Biomol. Spectrosc. 2010, 76 (2), 137-141. https://doi.org/10.1016/j.saa.2010.02.051.

(48) Kim, S. H.; Nyande, B. W.; Kim, H. S.; Park, J. S.; Lee, W. J.; Oh, M. Numerical Analysis of Thermal Decomposition for RDX, TNT, and Composition B. J. Hazard. Mater. 2016, 308, 120-130. https://doi.org/10.1016/j.jhazmat.2015.12.061.

(49) Kresse, G. Ab Initio Molecular Dynamics for Liquid Metals. J. Non. Cryst. Solids 1995, 192-193, 222-229. https://doi.org/10.1016/0022-3093(95)00355-X.

(50) Kresse, G.; Furthmüller, J. Efficiency of Ab-Initio Total Energy Calculations for Metals and Semiconductors Using a Plane-Wave Basis Set. Comput. Mater. Sci. 1996, 6 (1), 1550. https://doi.org/10.1016/0927-0256(96)00008-0.

(51) Kresse, G.; Joubert, D. From Ultrasoft Pseudopotentials to the Projector Augmented- 
Wave Method. Phys. Rev. B 1999, 59 (3), 1758-1775.

https://doi.org/10.1103/PhysRevB.59.1758.

(52) Plimpton, S. Fast Parallel Algorithms for Short-Range Molecular Dynamics. J. Comput. Phys. 1995, 117 (1), 1-19. https://doi.org/10.1006/jcph.1995.1039.

(53) Plimpton, S. J.; Thompson, A. P. Computational Aspects of Many-Body Potentials. MRS Bull. 2012, 37 (5), 513-521. https://doi.org/10.1557/mrs.2012.96.

(54) Blöchl, P. E. Projector Augmented-Wave Method. Phys. Rev. B 1994, 50 (24), 1795317979. https://doi.org/10.1103/PhysRevB.50.17953.

(55) Perdew, J. P.; Burke, K.; Ernzerhof, M. Generalized Gradient Approximation Made Simple. Phys. Rev. Lett. 1996, 77 (18), 3865-3868. https://doi.org/10.1103/PhysRevLett.77.3865.

(56) Methfessel, M.; Paxton, A. T. High-Precision Sampling for Brillouin-Zone Integration in Metals. Phys. Rev. B 1989, 40 (6), 3616-3621. https://doi.org/10.1103/PhysRevB.40.3616.

(57) Grimme, S.; Ehrlich, S.; Goerigk, L. Effect of the Damping Function in Dispersion Corrected Density Functional Theory. J. Comput. Chem. 2011, 32 (7), 1456-1465. https://doi.org/10.1002/jcc.21759.

(58) Togo, A.; Oba, F.; Tanaka, I. First-Principles Calculations of the Ferroelastic Transition between Rutile-Type and $\mathrm{CaCl}_{2}$-Type $\mathrm{SiO}_{2}$ at High Pressures. Phys. Rev. B 2008, 78 (13), 134106. https://doi.org/10.1103/PhysRevB.78.134106.

(59) Togo, A.; Tanaka, I. First Principles Phonon Calculations in Materials Science. Scr. 
Mater. 2015, 108, 1-5. https://doi.org/10.1016/j.scriptamat.2015.07.021.

(60) Skelton, J. M.; Burton, L. A.; Jackson, A. J.; Oba, F.; Parker, S. C.; Walsh, A. Lattice Dynamics of the Tin Sulphides $\mathrm{SnS}_{2}, \mathrm{SnS}$ and $\mathrm{Sn}_{2} \mathrm{~S}_{3}$ : Vibrational Spectra and Thermal Transport. Phys. Chem. Chem. Phys. 2017, 19 (19), 12452-12465. https://doi.org/10.1039/C7CP01680H.

(61) Stukowski, A. Visualization and Analysis of Atomistic Simulation Data with OVITO-the Open Visualization Tool. Model. Simul. Mater. Sci. Eng. 2010, 18 (1), 015012. https://doi.org/10.1088/0965-0393/18/1/015012.

(62) Togo, A.; Chaput, L.; Tanaka, I. Distributions of Phonon Lifetimes in Brillouin Zones. Phys. Rev. B 2015, 91 (9), 094306. https://doi.org/10.1103/PhysRevB.91.094306.

(63) Berens, P. H.; Mackay, D. H. J.; White, G. M.; Wilson, K. R. Thermodynamics and Quantum Corrections from Molecular Dynamics for Liquid Water. J. Chem. Phys. 1983, 79 (5), 2375-2389. https://doi.org/10.1063/1.446044.

(64) Boulard, B.; Kieffer, J.; Phifer, C. C.; Angell, C. A. Vibrational Spectra in Fluoride Crystals and Glasses at Normal and High Pressures by Computer Simulation. J. Non. Cryst. Solids 1992, 140, 350-358. https://doi.org/10.1016/S0022-3093(05)80795-1.

(65) Wood, M. A.; van Duin, A. C. T.; Strachan, A. Coupled Thermal and Electromagnetic Induced Decomposition in the Molecular Explosive AHMX; A Reactive Molecular Dynamics Study. J. Phys. Chem. A 2014, 118 (5), 885-895. https://doi.org/10.1021/jp406248m.

(66) Bedrov, D.; Hooper, J. B.; Smith, G. D.; Sewell, T. D. Shock-Induced Transformations in 
Crystalline RDX: A Uniaxial Constant-Stress Hugoniostat Molecular Dynamics

Simulation Study. J. Chem. Phys. 2009, 131 (3), 034712.

https://doi.org/10.1063/1.3177350.

(67) Schneider, T.; Stoll, E. Molecular-Dynamics Study of a Three-Dimensional OneComponent Model for Distortive Phase Transitions. Phys. Rev. B 1978, 17 (3), 13021322. https://doi.org/10.1103/PhysRevB.17.1302.

(68) Ceriotti, M.; Bussi, G.; Parrinello, M. Nuclear Quantum Effects in Solids Using a Colored-Noise Thermostat. Phys. Rev. Lett. 2009, 103 (3), 030603. https://doi.org/10.1103/PhysRevLett.103.030603.

(69) Ceriotti, M.; Bussi, G.; Parrinello, M. Colored-Noise Thermostats à La Carte. J. Chem. Theory Comput. 2010, 6 (4), 1170-1180. https://doi.org/10.1021/ct900563s.

(70) Dettori, R.; Ceriotti, M.; Hunger, J.; Melis, C.; Colombo, L.; Donadio, D. Simulating Energy Relaxation in Pump-Probe Vibrational Spectroscopy of Hydrogen-Bonded Liquids. J. Chem. Theory Comput. 2017, 13 (3), 1284-1292. https://doi.org/10.1021/acs.jctc.6b01108.

(71) Dettori, R.; Ceriotti, M.; Hunger, J.; Colombo, L.; Donadio, D. Energy Relaxation and Thermal Diffusion in Infrared Pump-Probe Spectroscopy of Hydrogen-Bonded Liquids. J. Phys. Chem. Lett. 2019, 10 (12), 3447-3452. https://doi.org/10.1021/acs.jpclett.9b01272.

(72) Polack, T. A Filtering Procedure for Systematic Removal of Pump-Perturbed Polarization Artifacts. Opt. Express 2006, 14 (12), 5823. https://doi.org/10.1364/oe.14.005823.

(73) Nuernberger, P.; Lee, K. F.; Bonvalet, A.; Polack, T.; Vos, M. H.; Alexandrou, A.; Joffre, 
M. Suppression of Perturbed Free-Induction Decay and Noise in Experimental Ultrafast Pump-Probe Data. Opt. Lett. 2009, 34 (20), 3226. https://doi.org/10.1364/OL.34.003226.

(74) Hamm, P. Coherent Effects in Femtosecond Infrared Spectroscopy. Chem. Phys. 1995, 200 (3), 415-429. https://doi.org/10.1016/0301-0104(95)00262-6.

(75) Yan, S.; Seidel, M. T.; Tan, H. S. Perturbed Free Induction Decay in Ultrafast Mid-IR Pump-Probe Spectroscopy. Chem. Phys. Lett. 2011, 517 (1-3), 36-40. https://doi.org/10.1016/j.cplett.2011.10.013.

(76) Lorenc, M.; Ziolek, M.; Naskrecki, R.; Karolczak, J.; Kubicki, J.; Maciejewski, A. Artifacts in Femtosecond Transient Absorption Spectroscopy. Appl. Phys. B Lasers Opt. 2002, 74 (1), 19-27. https://doi.org/10.1007/s003400100750.

(77) Mullen, K. M.; Stokkum, I. H. M. van. TIMP : An R Package for Modeling Multi-Way Spectroscopic Measurements. J. Stat. Softw. 2007, 18 (3). https://doi.org/10.18637/jss.v018.i03.

(78) Snellenburg, J. J.; Laptenok, S. P.; Seger, R.; Mullen, K. M.; Stokkum, I. H. M. van. Glotaran : A Java -Based Graphical User Interface for the R Package TIMP. J. Stat. Softw. 2012, 49 (3). https://doi.org/10.18637/jss.v049.i03.

(79) Skelton, J. M.; Jackson, A. J.; Dimitrievska, M.; Wallace, S. K.; Walsh, A. Vibrational Spectra and Lattice Thermal Conductivity of Kesterite-Structured $\mathrm{Cu}_{2} \mathrm{ZnSnS}_{4}$ and $\mathrm{Cu}_{2} \mathrm{ZnSnSe}_{4}$. APL Mater. 2015, 3 (4), 041102. https://doi.org/10.1063/1.4917044.

(80) Mukhopadhyay, S.; Parker, D. S.; Sales, B. C.; Puretzky, A. A.; McGuire, M. A.; Lindsay, L. Two-Channel Model for Ultralow Thermal Conductivity of Crystalline $\mathrm{Tl}_{3} \mathrm{VSe}_{4}$. 
Science (80-. ). 2018, 360 (6396), 1455-1458. https://doi.org/10.1126/science.aar8072.

(81) Mandal, A.; Ramasesha, K.; De Marco, L.; Tokmakoff, A. Collective Vibrations of Water-Solvated Hydroxide Ions Investigated with Broadband 2DIR Spectroscopy. $J$. Chem. Phys. 2014, 140 (20), 204508. https://doi.org/10.1063/1.4878490.

(82) Marcus, R. A. Unimolecular Dissociations and Free Radical Recombination Reactions. J. Chem. Phys. 1952, 20 (3), 359-364. https://doi.org/10.1063/1.1700424.

(83) Rosenstock, H. M.; Wallenstein, M. B.; Wahrhaftig, A. L.; Eyring, H. Absolute Rate Theory for Isolated Systems and the Mass Spectra of Polyatomic Molecules. Proc. Natl. Acad. Sci. 1952, 38 (8), 667-678. https://doi.org/10.1073/pnas.38.8.667.

(84) Graener, H.; Laubereau, A. Ultrafast Vibrational Energy Transfer of Polyethylene Investigated with Picosecond Laser Pulses. Chem. Phys. Lett. 1987, 133 (5), 378-380. https://doi.org/10.1016/0009-2614(87)87086-0.

(85) Fried, L. E.; Ruggiero, A. J. Energy Transfer Rates in Primary, Secondary, and Insensitive Explosives. J. Phys. Chem. 1994, 98 (39), 9786-9791. https://doi.org/10.1021/j100090a012.

(86) Chen, S.; Hong, X.; Hill, J. R.; Dlott, D. D. Ultrafast Energy Transfer in High Explosives: Vibrational Cooling. J. Phys. Chem. 1995, 99 (13), 4525-4530. https://doi.org/10.1021/j100013a023.

(87) Greetham, G. M.; Clark, I. P.; Young, B.; Fritsch, R.; Minnes, L.; Hunt, N. T.; Towrie, M. Time-Resolved Temperature-Jump Infrared Spectroscopy at a High Repetition Rate. Appl. Spectrosc. 2020, 74 (6), 720-727. https://doi.org/10.1177/0003702820913636. 
(88) Kim, H.; Dlott, D. D. Theory of Ultrahot Molecular Solids: Vibrational Cooling and Shock-Induced Multiphonon up Pumping in Crystalline Naphthalene. J. Chem. Phys. 1990, 93 (3), 1695-1709. https://doi.org/10.1063/1.459097.

(89) Schweigert, I. V. Ab Initio Molecular Dynamics of High-Temperature Unimolecular Dissociation of Gas-Phase RDX and Its Dissociation Products. J. Phys. Chem. A 2015, 119 (12), 2747-2759. https://doi.org/10.1021/jp510034p.

(90) Sakano, M. N.; Hamed, A.; Kober, E. M.; Grilli, N.; Hamilton, B. W.; Islam, M. M.; Koslowski, M.; Strachan, A. Unsupervised Learning-Based Multiscale Model of Thermochemistry in 1,3,5-Trinitro-1,3,5-Triazinane (RDX). J. Phys. Chem. A 2020, 124 (44), 9141-9155. https://doi.org/10.1021/acs.jpca.0c07320.

(91) Nomura, K.; Kalia, R. K.; Nakano, A.; Vashishta, P.; van Duin, A. C. T.; Goddard, W. A. Dynamic Transition in the Structure of an Energetic Crystal during Chemical Reactions at Shock Front Prior to Detonation. Phys. Rev. Lett. 2007, 99 (14), 148303. https://doi.org/10.1103/PhysRevLett.99.148303.

(92) Cai, Y.; Zhao, F. P.; An, Q.; Wu, H. A.; Goddard, W. A.; Luo, S. N. Shock Response of Single Crystal and Nanocrystalline Pentaerythritol Tetranitrate: Implications to Hotspot Formation in Energetic Materials. J. Chem. Phys. 2013, 139 (16), 164704. https://doi.org/10.1063/1.4825400.

(93) Park, S. D.; Armstrong, M. R.; Kohl, I. T.; Zaug, J. M.; Knepper, R.; Tappan, A. S.; Bastea, S.; Kay, J. J. Ultrafast Shock-Induced Reactions in Pentaerythritol Tetranitrate Thin Films. J. Phys. Chem. A 2018, 122 (41), 8101-8106. https://doi.org/10.1021/acs.jpca.8b05387. 
(94) Tarver, C. M.; Chidester, S. K.; Nichols, A. L. Critical Conditions for Impact- and ShockInduced Hot Spots in Solid Explosives. J. Phys. Chem. 1996, 100 (14), 5794-5799. https://doi.org/10.1021/jp953123s.

(95) Henson, B. F. Ignition Chemistry in HMX from Thermal Explosion to Detonation. In AIP Conference Proceedings; AIP, 2002; Vol. 620, pp 1069-1072. https://doi.org/10.1063/1.1483723.

(96) BALZER, J.; FIELD, J.; GIFFORD, M.; PROUD, W.; WALLEY, S. High-Speed Photographic Study of the Drop-Weight Impact Response of Ultrafine and Conventional PETN and RDX. Combust. Flame 2002, 130 (4), 298-306. https://doi.org/10.1016/S00102180(02)00373-5.

(97) Zeman, S.; Jungová, M. Sensitivity and Performance of Energetic Materials. Propellants, Explos. Pyrotech. 2016, 41 (3), 426-451. https://doi.org/10.1002/prep.201500351. 


\section{Supporting Information}

\section{Mode-Selective Vibrational Energy Transfer Dynamics in 1,3,5-Trinitroperhydro-1,3,5-Triazine (RDX) Thin Films}

Neil C. Cole-Filipiak ${ }^{1}$, Robert Knepper ${ }^{2}$, Mitchell Wood ${ }^{2, *}$, and Krupa Ramasesha ${ }^{1, *}$

${ }^{1}$ Combustion Research Facility, Sandia National Laboratories, Livermore, CA 94550, USA

${ }^{2}$ Sandia National Laboratories, Albuquerque, NM 87185, USA

*Electronic mail: kramase@sandia.gov, mitwood@sandia.gov.

\section{S1 RDX Sample Characterization}

a)

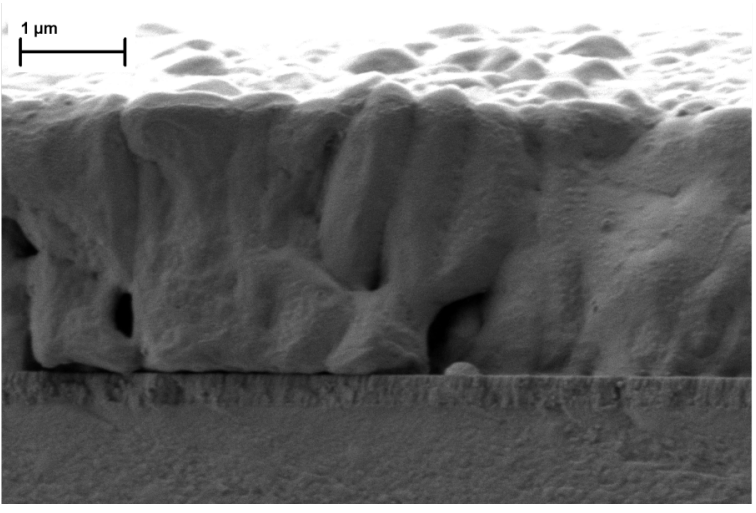

b)

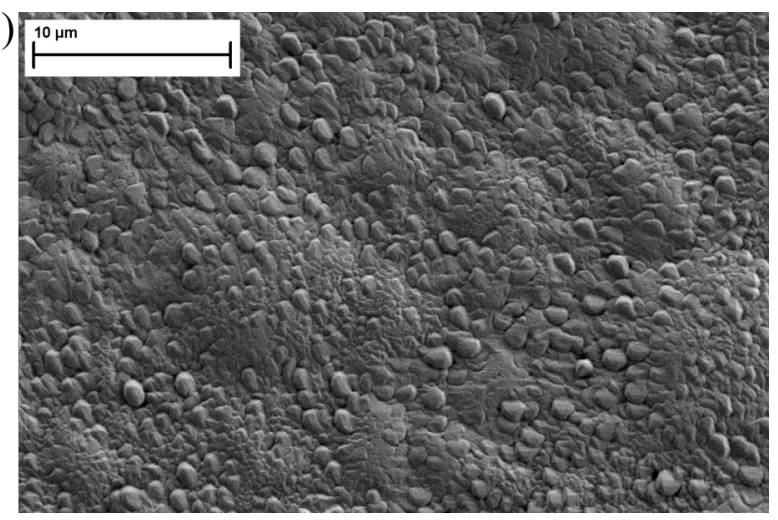

Figure S1: a) Fracture cross-section and b) surface morphology of RDX vapor deposited onto BK-7. The RDX films display a columnar morphology with columns oriented perpendicular to the surface. The surface itself reveals hillocks $(\leq 10 \mu \mathrm{m}$ lateral size and approximately 100-200 nm tall) composed of smaller RDX grains (ca. 0.5 to $1 \mu \mathrm{m}$ ). 


\section{S2 Additional Theoretical Results}

\section{S2.1 Vibrational Modes of Crystalline RDX}

Table S1: Vector displacement diagram of the major RDX modes, calculated and experimental frequencies, and mode assignments. Calculated frequencies in bold are shown as the example vector displacement diagram, values in parentheses represent a $10 \%$ compressed crystal along the [010] axis. The equatorial $\mathrm{NO}_{2}$ group is labeled " $\mathrm{E}$ " in each unique view.

\begin{tabular}{|c|c|c|c|}
\hline $\begin{array}{c}\text { Example Vector } \\
\text { Diagram }\end{array}$ & $\begin{array}{c}\text { Calculated } \\
\text { Frequency } \\
\left(\mathrm{cm}^{-1}\right)\end{array}$ & $\begin{array}{c}\text { Experimental } \\
\text { Frequency } \\
\left(\mathrm{cm}^{-1}\right)\end{array}$ & Assignment \\
\hline & $\begin{array}{c}1626,1625, \\
1616,1596, \\
\mathbf{1 5 9 4}(1780, \\
1776,1770, \\
1737,1734)\end{array}$ & $\sim 1590$ & $\begin{array}{l}\mathrm{NO}_{2}{ }^{(\mathrm{ax})} \\
\text { asymmetric } \\
\text { stretch }\end{array}$ \\
\hline & $\begin{array}{l}1562, \mathbf{1 5 5 2} \\
(1648,1643)\end{array}$ & 1540 & $\begin{array}{c}\mathrm{NO}_{2}{ }^{(\mathrm{eq})} \\
\text { asymmetric } \\
\text { stretch }\end{array}$ \\
\hline & $\begin{array}{l}\text { 1432, 1424, } \\
1412(1557 \\
1441,1440)\end{array}$ & 1460,1420 & $\begin{array}{c}\mathrm{CH}_{2} \text { scissor, } \\
\mathrm{N}-\mathrm{N} \text { stretch, } \\
\mathrm{NO}_{2} \\
\text { bend/symmetric } \\
\text { stretch }\end{array}$ \\
\hline
\end{tabular}




\begin{tabular}{|c|c|c|c|}
\hline $\begin{array}{c}\text { Example Vector } \\
\text { Diagram }\end{array}$ & $\begin{array}{l}\text { Calculated } \\
\text { Frequencies } \\
\quad\left(\mathrm{cm}^{-1}\right)\end{array}$ & $\begin{array}{c}\text { Experimental } \\
\text { Frequency } \\
\left(\mathrm{cm}^{-1}\right)\end{array}$ & Assignment \\
\hline & $\begin{array}{c}1398,1395, \\
1388,1380, \\
1375(1400, \\
1399,1376, \\
1371)\end{array}$ & 1390 & $\begin{array}{c}\mathrm{CH}_{2} \text { scissor, } \\
\mathrm{CH}_{2} \text { bend, } \mathrm{N}-\mathrm{N} \\
\text { stretch }\end{array}$ \\
\hline & $\begin{array}{c}1344,1342, \\
1336, \mathbf{1 3 3 1}, \\
1320,1317,1308 \\
(1364,1362, \\
1341,1337, \\
1326,1324, \\
1308)\end{array}$ & 1350,1320 & $\begin{array}{c}\mathrm{CH}_{2} \text { scissor, } \\
\mathrm{CH}_{2} \text { bend, } \mathrm{N}-\mathrm{N} \\
\text { stretch }\end{array}$ \\
\hline & $\begin{array}{c}1286,1270, \\
\mathbf{1 2 6 8}, 1258, \\
1243,1243 \\
(1307,1288, \\
1284,1272, \\
1261)\end{array}$ & $\sim 1270$ & $\begin{array}{l}\mathrm{N}-\mathrm{N}^{(\mathrm{eq})} \text { stretch, } \\
\mathrm{CH}_{2} \text { bend, } \mathrm{NO}_{2} \\
\text { bend/symmetric } \\
\text { stretch }\end{array}$ \\
\hline & $\begin{array}{l}\mathbf{1 2 4 0}, 1239, \\
1235(1257, \\
1245,1244)\end{array}$ & 1230,1220 & $\begin{array}{l}\text { ring } \mathrm{C}-\mathrm{N}-\mathrm{C} \\
\text { stretch, } \mathrm{CH}_{2} \\
\text { bend }\end{array}$ \\
\hline & $\begin{array}{c}1064, \mathbf{1 0 6 0} \\
(1101,1091)\end{array}$ & 1040 & $\begin{array}{c}\mathrm{N}-\mathrm{N}^{(\text {eq })} \text { stretch, } \\
\mathrm{NO}_{2} \\
\text { bend/symmetric } \\
\text { stretch, ring } \\
\mathrm{C}-\mathrm{N}-\mathrm{C} \\
\text { stretch, } \mathrm{CH}_{2} \\
\text { twist, } \mathrm{CH}_{2} \text { bend }\end{array}$ \\
\hline
\end{tabular}




\begin{tabular}{|c|c|c|c|}
\hline $\begin{array}{c}\text { Example Vector } \\
\text { Diagram }\end{array}$ & $\begin{array}{l}\text { Calculated } \\
\text { Frequencies } \\
\quad\left(\mathrm{cm}^{-1}\right)\end{array}$ & $\begin{array}{l}\text { Experimental } \\
\text { Frequency } \\
\left(\mathrm{cm}^{-1}\right)\end{array}$ & Assignment \\
\hline & 1000 (1014) & 1010 & $\begin{array}{c}\mathrm{N}-\mathrm{N}^{(\mathrm{ax})} \text { stretch, } \\
\mathrm{CH}_{2} \text { twist, } \mathrm{CH}_{2} \\
\text { wag }\end{array}$ \\
\hline & $\begin{array}{c}\text { 946, } 944(961 \\
960)\end{array}$ & 945 & $\begin{array}{c}\mathrm{N}-\mathrm{N} \text { stretch, } \\
\mathrm{CH}_{2} \text { wag, } \mathrm{NO}_{2} \\
\text { bend, ring } \\
\text { breathing }\end{array}$ \\
\hline & $\begin{array}{c}910, \mathbf{9 0 8}, 893 \\
892(928,927 \\
906,905)\end{array}$ & 930 & $\begin{array}{l}\text { ring distortion, } \\
\mathrm{CH}_{2} \text { wag, } \mathrm{NO}_{2} \\
\text { bend }\end{array}$ \\
\hline & $\begin{array}{c}882,876 \\
892)\end{array}(893$, & 880 & $\begin{array}{c}\text { out-of-plane } \\
\text { ring distortion, } \\
\mathrm{CH}_{2} \text { wag, } \mathrm{N}-\mathrm{N} \\
\text { stretch, } \mathrm{C}-\mathrm{N} \\
\text { stretch, } \mathrm{NO}_{2} \\
\text { bend }\end{array}$ \\
\hline & $846(860)$ & 840 & $\begin{array}{l}\text { in-plane ring } \\
\text { distortion, } \mathrm{NO}_{2} \\
\text { bend, } \mathrm{N}-\mathrm{N} \\
\text { stretch }\end{array}$ \\
\hline
\end{tabular}




\section{S2.2 Two-Phonon Density of States}

Part of our theory support for the experimentally observed VET comes from the analysis of the calculated two-phonon density of states where select mode populations have been modified to "pump" a vibration of interest $(\Delta 2 \mathrm{PhDoS})$. These data reveal which modes are coupled based on the selection rules of momentum conservation and allowable wavevectors within the Brillouin zone. Calculated $\Delta 2 \mathrm{PhDoS}$ between thermal occupation, i.e. $n_{\lambda}=$ BoseEinstein, and an additional Gaussian perturbed population at specific pump targeted modes are given in Fig. S2. Each of the $\Delta 2 \mathrm{PhDoS}$ spectra are summed over all sampled Brillouin zone points $(5 \times 5 \times 5 \mathrm{mesh})$. These $\Delta 2 \mathrm{PhDoS}$ can be interpreted as the change in scattering intensity when the pump has been applied to the system: a non-zero value occurs when a mode is coupled to the pumped mode and any frequencies with $\Delta 2 \mathrm{PhDoS}=0$ have no change relative to a system in equilibrium. In other words, any frequency plotted that exhibits a non-zero $\triangle 2 \mathrm{PhDoS}$ is coupled to the pumped mode given as the data series label. Given the infrared spectrum overlaid with this scattering intensity data, it is clear that there are numerous allowable two-phonon collisions that couple all native RDX modes together. Missing in these data - to complete the experimental to theory connection - are the crosssection (strength of mode-to-mode coupling) of each of the allowable transition, as discussed in the main text.

Contained in Fig. S2 are DFT predictions of the static IR spectrum (reproduced from Fig. 1) and $\Delta 2 \mathrm{PhDoS}$ for RDX; panels differ only in the two-phonon density of states with the pumped mode, see mode assignments in Table S1. An overlap of both data series is needed to identify vibrations which are both coupled to the pumped mode and experimentally observable. Further details regarding the calculation and interpretation of these $\Delta 2 \mathrm{PhDoS}$ have been detailed previously. ${ }^{1}$ 

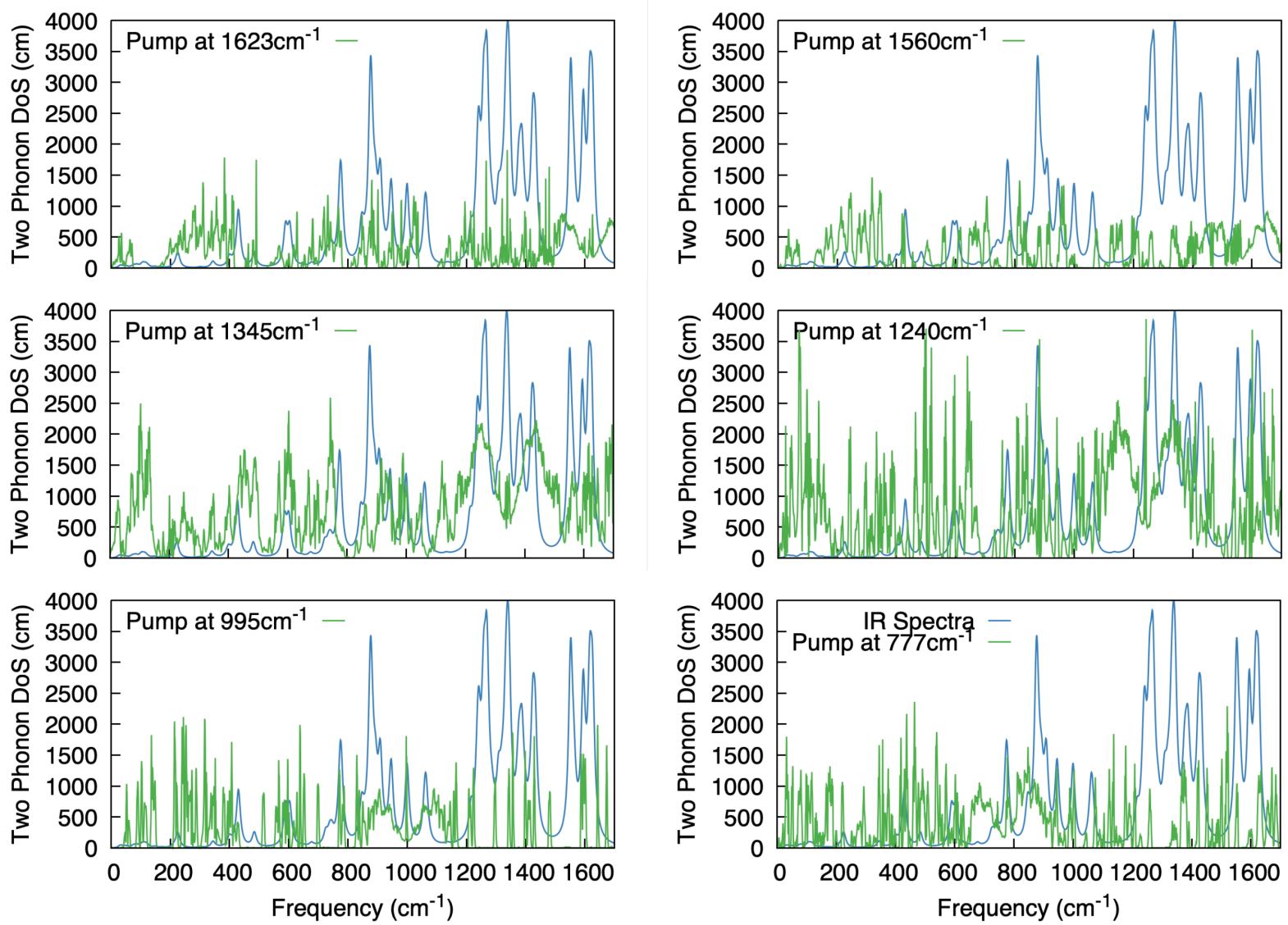

Figure S2: Two-phonon density of states prediction from VASP DFT calculations following an anharmonic (third-order) expansion of the potential energy surface of the ions. Plotted as the difference from a thermal equilibrium state (i.e. $\Delta 2 \mathrm{PhDoS}$ ), each calculation perturbs the occupation of specific modes to highlight coupling to this artificially pumped vibration. The Phonopy and Phono3py software packages are used to collect the finite displacement method calculations from VASP. 


\section{S2.3 MD Simulations}

For these classical simulations in equilibrium, each vibration of the material will carry an equipartition of the kinetic energy $\left(\frac{1}{2} k_{b} T\right)$, regardless of the mode frequency. All MD simulations are evolved through a finite timestep integration given some prescription of the thermodynamic ensemble. For example, coupling to an external thermal bath to maintain control of the temperature constitutes a simulation in the canonical ensemble, and these adjustments to the equations of motion are colloquially known as "thermostats." Alternatively, an isolated system without a thermostat evolves in the micro-canonical ensemble, the ideal ensemble from which to sample vibrational spectra.

Additional SMRT fits and 2D spectra for frequency ranges containing the pumped mode, Figures S3 and S4, as well as low frequency modes that demonstrate elongated relaxation times in compressed RDX, Fig. S5. It is important to stress that the fitted SMRT are going to be most sensitive to the initial relaxation process as opposed to population redistribution toward the end of the simulation. While we do expect the evolving non-equilibrium phonon modes to perturb higher lying vibrations, as was observed in experiment, the effect will be subtle ( $<10 \%$ population changes) compared to the initial non-equilibrium state that pushes pumped (and coupled) modes up to twice an equipartition of energy. Future work will need to apply a similar fitting procedure as the experimental mid-IR data, allowing for multiple distinct timescales of relaxation to be extracted from the MD data. 

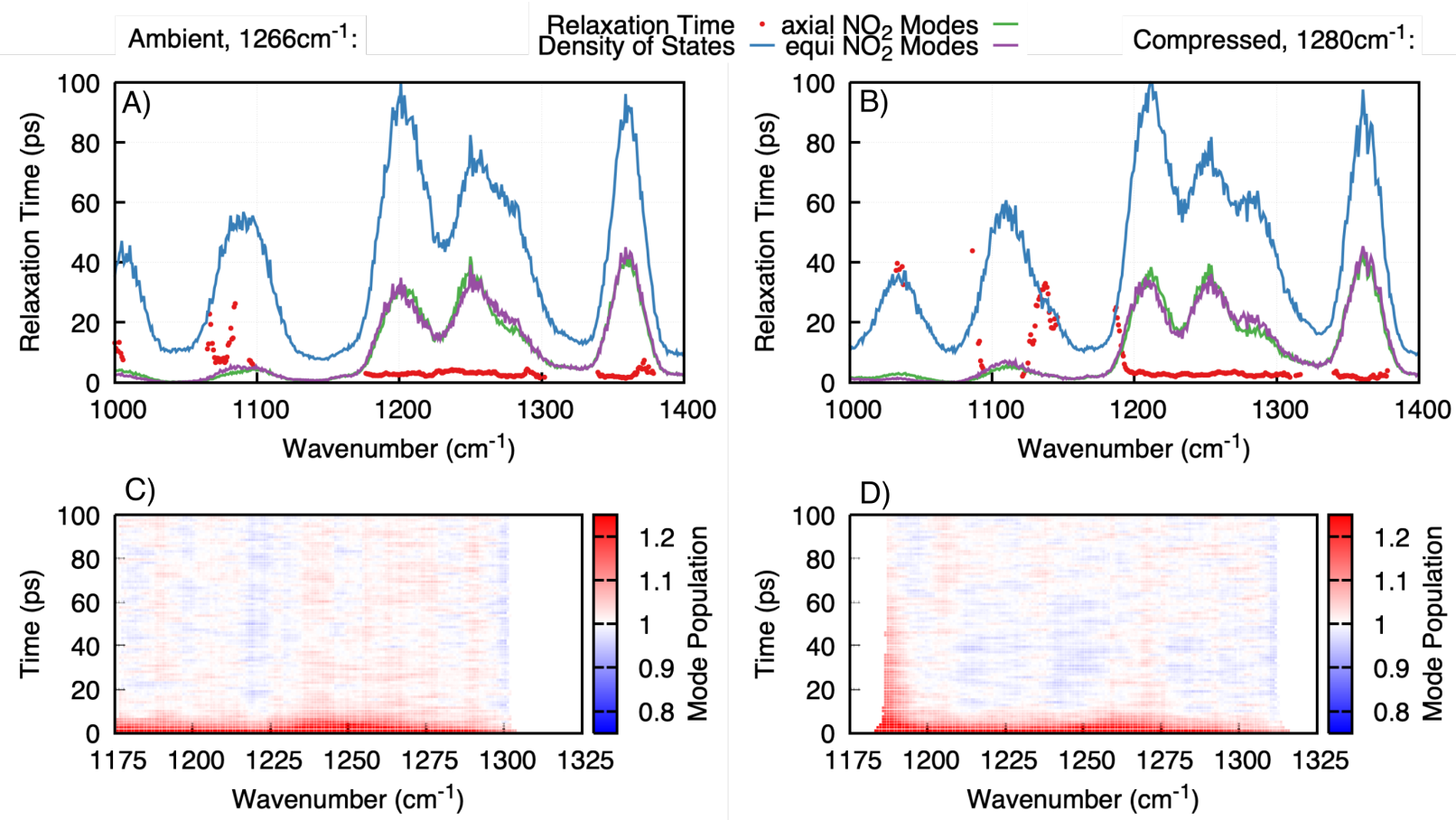

Figure S3: Pumped vibration is the $\mathrm{N}-\mathrm{N}^{(e q)}$ stretch, $\mathrm{CH}_{2}$ bend, and $\mathrm{NO}_{2}$ bend/symmetric stretch mode $\left(1260 \mathrm{~cm}^{-1}\right.$, ambient pressure) in both sets of data, time origin is where the pump thermostat is removed from the probe volume. (a) Extracted exponential decay time constants as the uncompressed RDX crystal returns to an equipartition of energy among all vibrations. Total density of states (blue) and partial density of sates (green, purple) highlight modes with axial versus equatorial $\mathrm{NO}_{2}$ character. (b) Time constants per-mode for a 10\% [010] compressed sample of RDX. Two-dimensional density of states normalized to an equipartition of energy (labeled as Mode Population) for (c) ambient and (d) compressed RDX; blue regions are under-populated and red over-populated with respect to the spectra at the same temperature. Frequency range shown highlights the pumped modes, and how there is no significant change in SMRT with compression. Fitted SMRT with high error are omitted from (a) and (b), while data is omitted from (c) and (d) at each minimum of the total density of states to avoid over-analyzing weak signal changes. 

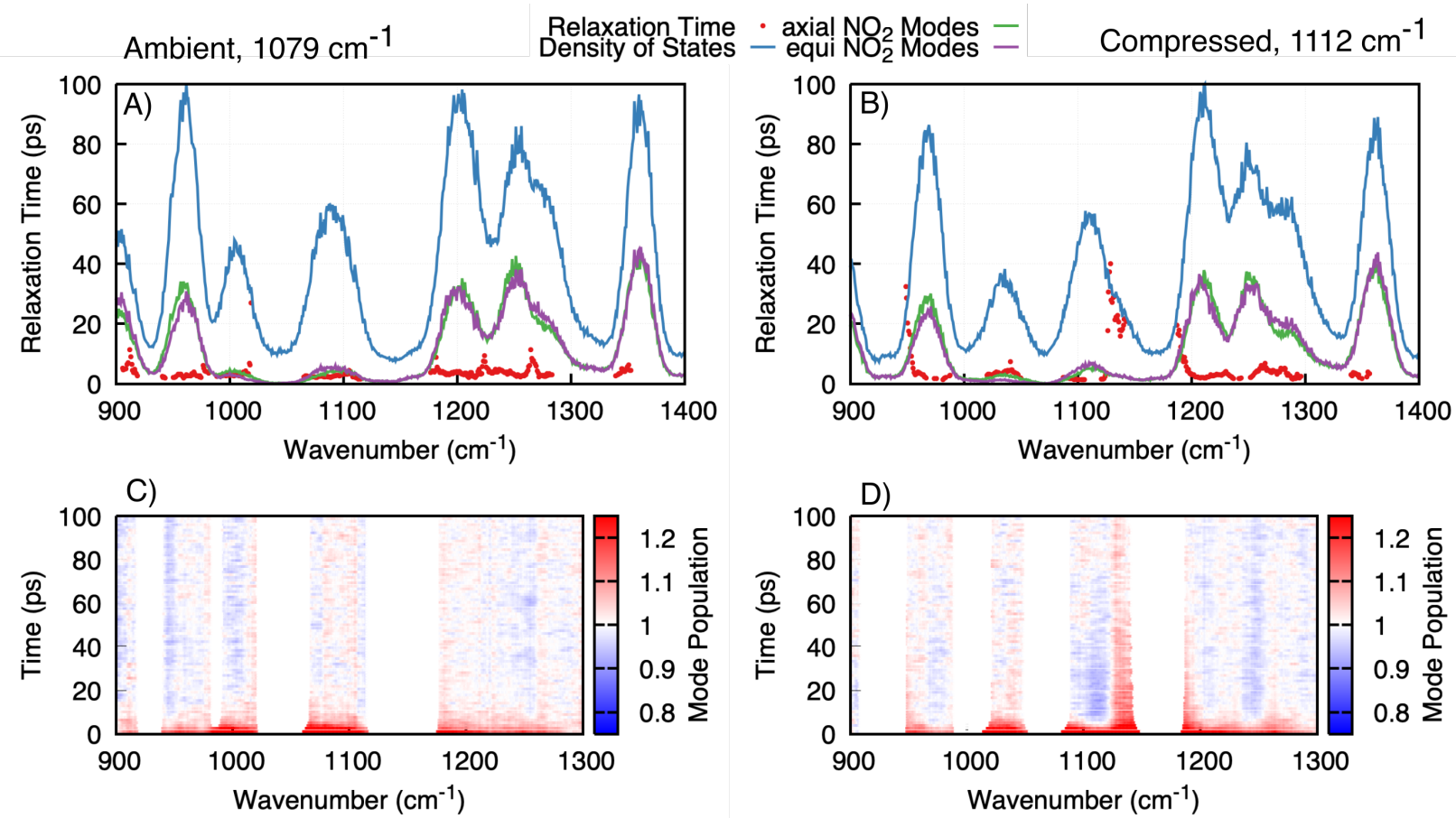

Figure S4: Pumped vibration is the combination of $\mathrm{N}-\mathrm{N}^{(\mathrm{eq})}$ stretch, $\mathrm{NO}_{2}$ bend/symmetric stretch, ring $\mathrm{C}-\mathrm{N}-\mathrm{C}$ stretch, $\mathrm{CH}_{2}$ twist, $\mathrm{CH}_{2}$ bend in both sets of data, time origin is where the pump thermostat is removed from the probe volume. Pumped mode has weak $\mathrm{NO}_{2}$ character, as seen in (a) and (b), and neither ambient nor compressed system shows significant evolution beyond the $\sim 10$ ps timescale. Shifting and broadening of peaks causes edge frequencies to central peaks to be spuriously fit with longer time dynamics than may be actually present. 

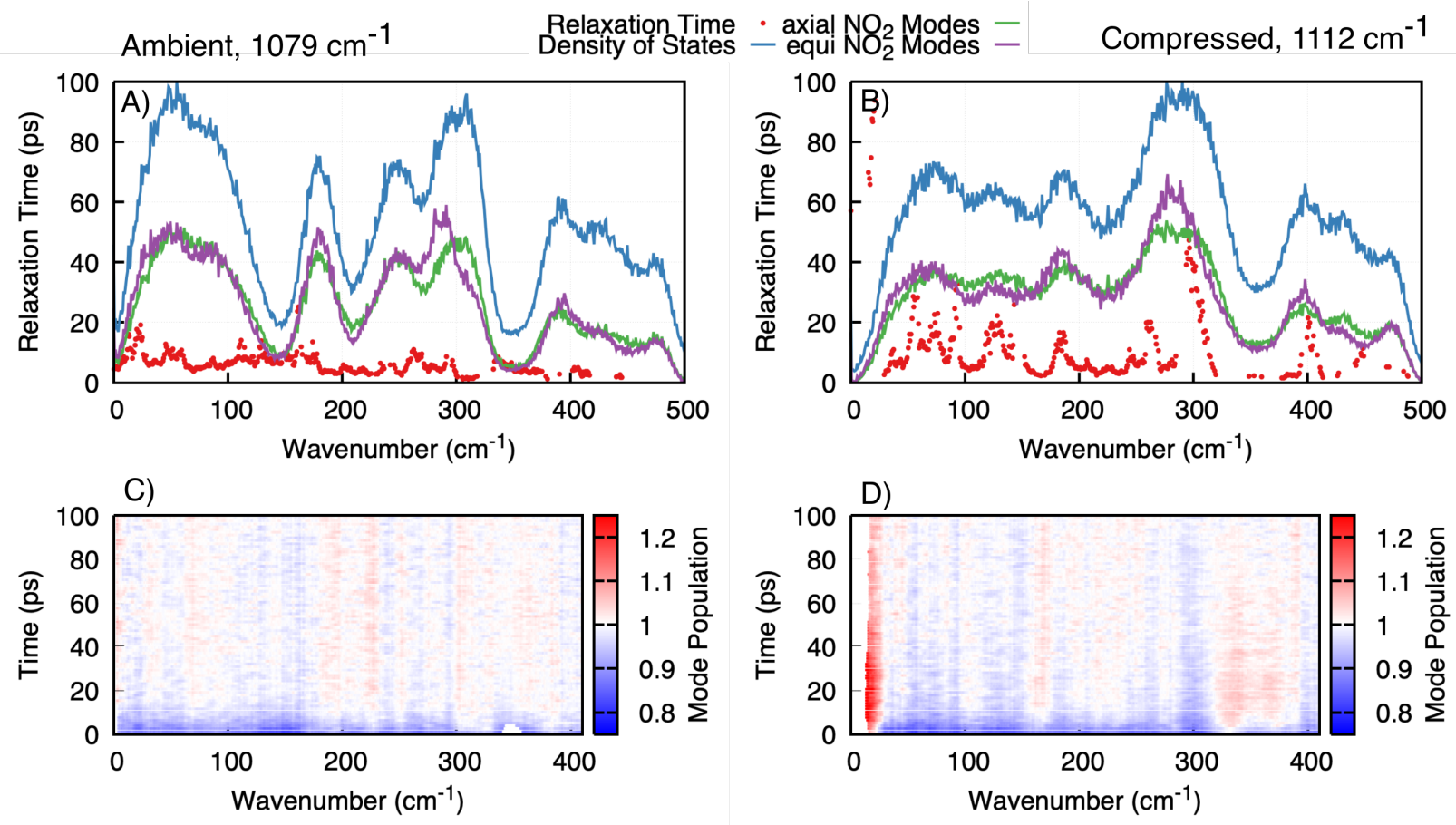

Figure S5: Pumped vibration is the combination of $\mathrm{N}-\mathrm{N}^{(\mathrm{eq})}$ stretch, $\mathrm{NO}_{2}$ bend/symmetric stretch, ring $\mathrm{C}-\mathrm{N}-\mathrm{C}$ stretch, $\mathrm{CH}_{2}$ twist, $\mathrm{CH}_{2}$ bend in both sets of data, time origin is where the pump thermostat is removed from the probe volume. As with other pumped high frequency modes, the SMRT of these low frequency modes show much longer time evolution where the sample is compressed. 


\section{S2.4 Mode-Selectivity of the 200 ps Evolution}

Table S2: Calculated harmonic modes of $\alpha$-RDX with IR intensities $\geq 0.1 \mathrm{e}^{2} \mathrm{amu}^{-1}$. Modes within the experimental probe window are then assigned a yes/no/neutral value for evolving with the 200 ps time constant; see main text for details.

\begin{tabular}{|c|c|c|}
\hline $\begin{array}{l}\text { Calc. Freq. } \\
\quad\left(\mathrm{cm}^{-1}\right)\end{array}$ & $\begin{array}{c}\text { Calc. IR } \\
\text { Intensity } \\
\left(\mathrm{e}^{2} \mathrm{amu}^{-1}\right)\end{array}$ & $\begin{array}{c}\text { Expt. } 200 \mathrm{ps} \\
\text { Evolution? }\end{array}$ \\
\hline 226 & 0.191 & - \\
\hline 430 & 0.289 & - \\
\hline 430 & 0.536 & - \\
\hline 485 & 0.148 & - \\
\hline 589 & 0.469 & - \\
\hline 604 & 0.325 & - \\
\hline 605 & 0.165 & - \\
\hline 725 & 0.144 & - \\
\hline 738 & 0.141 & - \\
\hline 746 & 0.111 & - \\
\hline 774 & 0.977 & - \\
\hline 778 & 0.573 & - \\
\hline 846 & 0.424 & no \\
\hline 876 & 2.455 & yes \\
\hline 882 & 0.507 & yes \\
\hline 892 & 0.171 & neutral \\
\hline 893 & 0.488 & no \\
\hline 908 & 0.686 & no \\
\hline 910 & 0.493 & no \\
\hline 944 & 0.316 & yes \\
\hline 946 & 0.793 & yes \\
\hline 1000 & 1.137 & neutral \\
\hline 1060 & 0.590 & yes \\
\hline 1065 & 0.539 & yes \\
\hline 1215 & 0.288 & yes \\
\hline 1235 & 0.216 & no \\
\hline 1239 & 0.229 & no \\
\hline 1240 & 0.859 & no \\
\hline 1243 & 0.233 & no \\
\hline 1243 & 0.286 & no \\
\hline 1258 & 1.724 & yes \\
\hline 1268 & 1.185 & yes \\
\hline 1270 & 1.188 & yes \\
\hline
\end{tabular}




\begin{tabular}{|c|c|c|}
\hline Calc. Freq. & IR Int. & $200 \mathrm{ps} ?$ \\
\hline 1286 & 0.215 & yes \\
\hline 1308 & 0.648 & neutral \\
\hline 1317 & 0.155 & yes \\
\hline 1321 & 0.285 & yes \\
\hline 1331 & 0.685 & neutral \\
\hline 1336 & 1.398 & no \\
\hline 1342 & 1.168 & no \\
\hline 1345 & 0.857 & no \\
\hline 1375 & 0.691 & neutral \\
\hline 1380 & 0.433 & no \\
\hline 1388 & 1.121 & no \\
\hline 1395 & 0.110 & no \\
\hline 1398 & 0.138 & no \\
\hline 1413 & 0.107 & no \\
\hline 1424 & 1.531 & no \\
\hline 1432 & 1.417 & neutral \\
\hline 1552 & 2.630 & no \\
\hline 1562 & 0.692 & no \\
\hline 1594 & 1.984 & yes \\
\hline 1597 & 0.110 & yes \\
\hline 1616 & 1.913 & yes \\
\hline 1625 & 1.695 & yes \\
\hline 1626 & 0.210 & yes \\
\hline 3025 & 0.364 & - \\
\hline 3075 & 0.181 & - \\
\hline 3075 & 0.739 & - \\
\hline 3095 & 0.264 & - \\
\hline 3107 & 0.354 & - \\
\hline
\end{tabular}



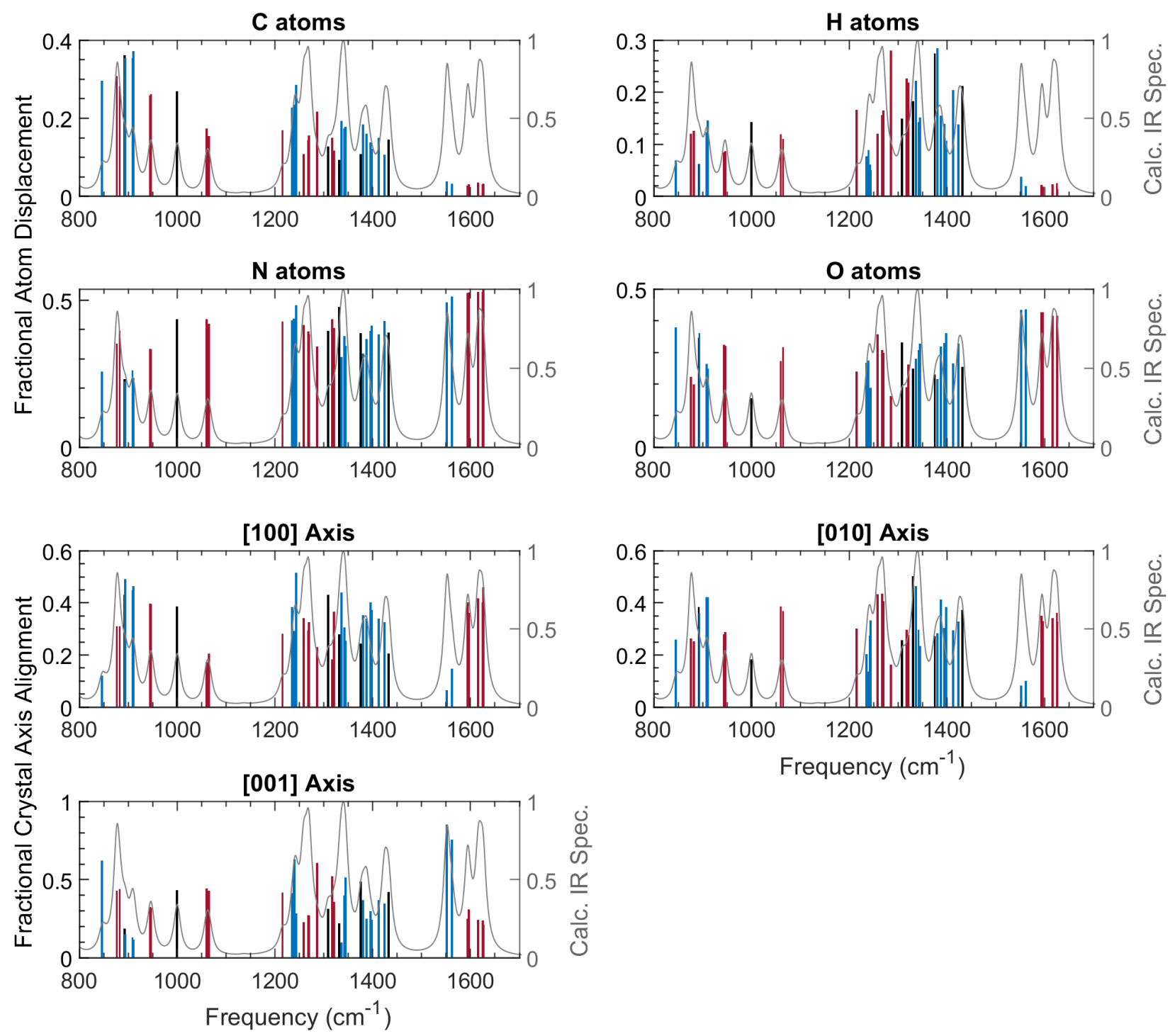

Figure S6: Fraction of each harmonic mode associated with each atom type (top four plots) or projected onto each crystal axis (bottom three plots) averaged over all eight RDX molecules in the unit cell. Bar height reports fraction while bar color highlights frequencies which experimentally demonstrate 200 ps evolution: red = "yes," blue = "no," and black = "neutral." Also plotted is the normalized, calculated IR spectrum from Fig. 1. 

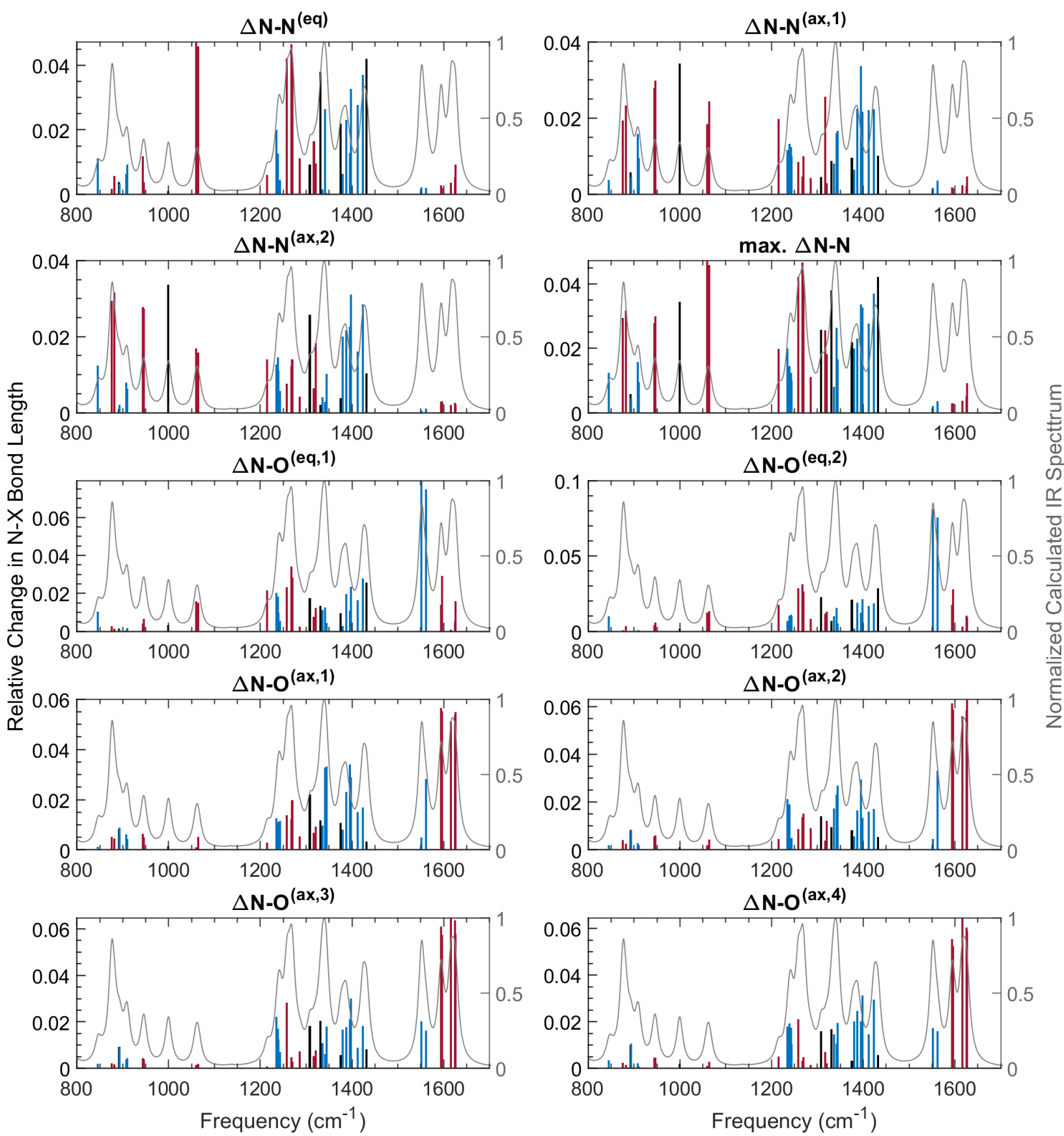

Figure S7: Fractional change of each $\mathrm{N}-\mathrm{X}$ bond length for each frequency averaged over all eight RDX molecules in the unit cell. Bar height reports fraction while bar color highlights frequencies which experimentally demonstrate 200 ps evolution: red = "yes," blue = "no," and black = "neutral." Also plotted is the normalized, calculated IR spectrum from Fig. 1. 

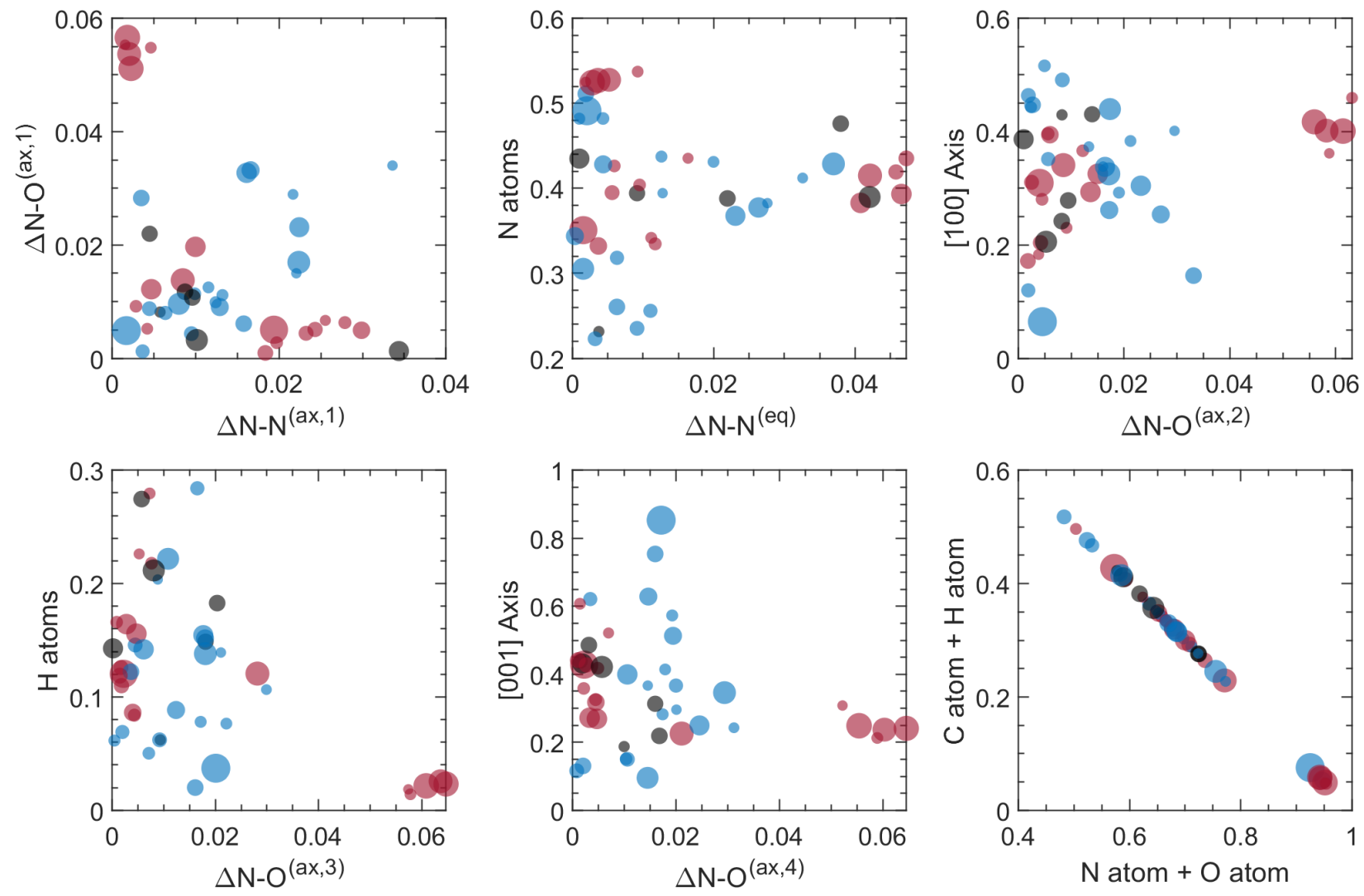

Figure S8: Example correlation plots comparing the relative fractional changes in $\mathrm{N}-\mathrm{X}$ bond lengths for each frequency. Marker size scales with calculated IR intensity while marker color highlights frequencies which experimentally demonstrate 200 ps evolution: red = "yes," blue $=$ "no," and black = "neutral." 


\section{S3 Additional Experimental Results}

The full set of all transient spectra are shown as false-color surface plots in Figures 2, 3, S9 and S11. These plots visualize changes in the transient absorption signal as a function of time-delay ( $x$ axis) and frequency ( $y$ axis) by mapping spectral intensity to the color coordinate. For example, in Fig. 2 in the main manuscript, a value of $0 \Delta \mathrm{mOD}$ has been assigned to white; blue corresponds to negative, bleach features (due to stimulated emission or loss of ground state absorption); and positive, transient absorption (TA) features appear in red. Vibrational excitation of isolated frequencies at early time-delays results in bleach/TA pairs which appear as parallel features of different colors. Dynamics, such as decay of an excitation, appear as a change in color towards the baseline, $0 \Delta \mathrm{mOD}$ value. While the colormap used in Figures 2 and 3 is useful for highlighting sign changes, other features such as intensity changes may not be clear. To help visualize all of the transient features, Figures 2 and 3 have been reproduced in Figures S9 and S11 using an alternate colormap.

Two thermal difference infrared spectra are shown in Fig. S14, which may be calculated as follows:

$$
-\log _{10}\left(\frac{\text { RDX transmission spectrum at } T_{2}}{\text { RDX transmission spectrum at } T_{1}}\right)
$$

with temperatures $T_{2}>T_{1}$. These thermal difference spectra highlight changes to the vibrational spectrum due to sample heating (i.e. exciting a higher energy phonon population distribution). The qualitative similarity of the positive/negative signal couplets in the thermal difference spectrum and transient specta at $\Delta t \geq 10 \mathrm{ps}$ - shown in Figures 4 , S10, and S12 - suggests that dynamics within the excited phonon bath are responsible for the longest observed dynamics. 

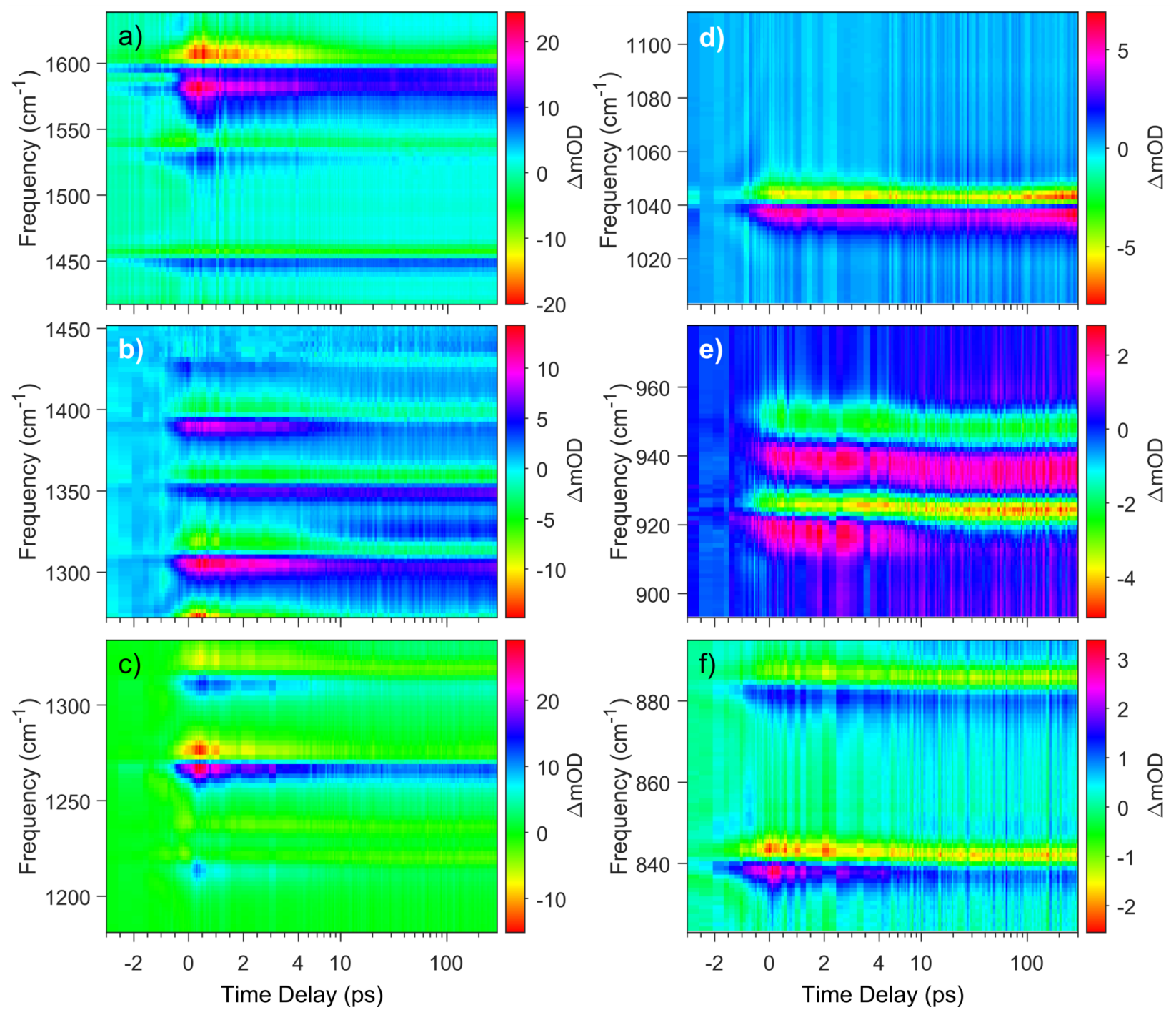

Figure S9: Reproduction of Fig. $2\left(1530 \mathrm{~cm}^{-1}\right.$ pump) using an alternate colormap; singularities are shown in white. Time-delays are shown in linear steps up to 4 ps and logarithmic thereafter. 

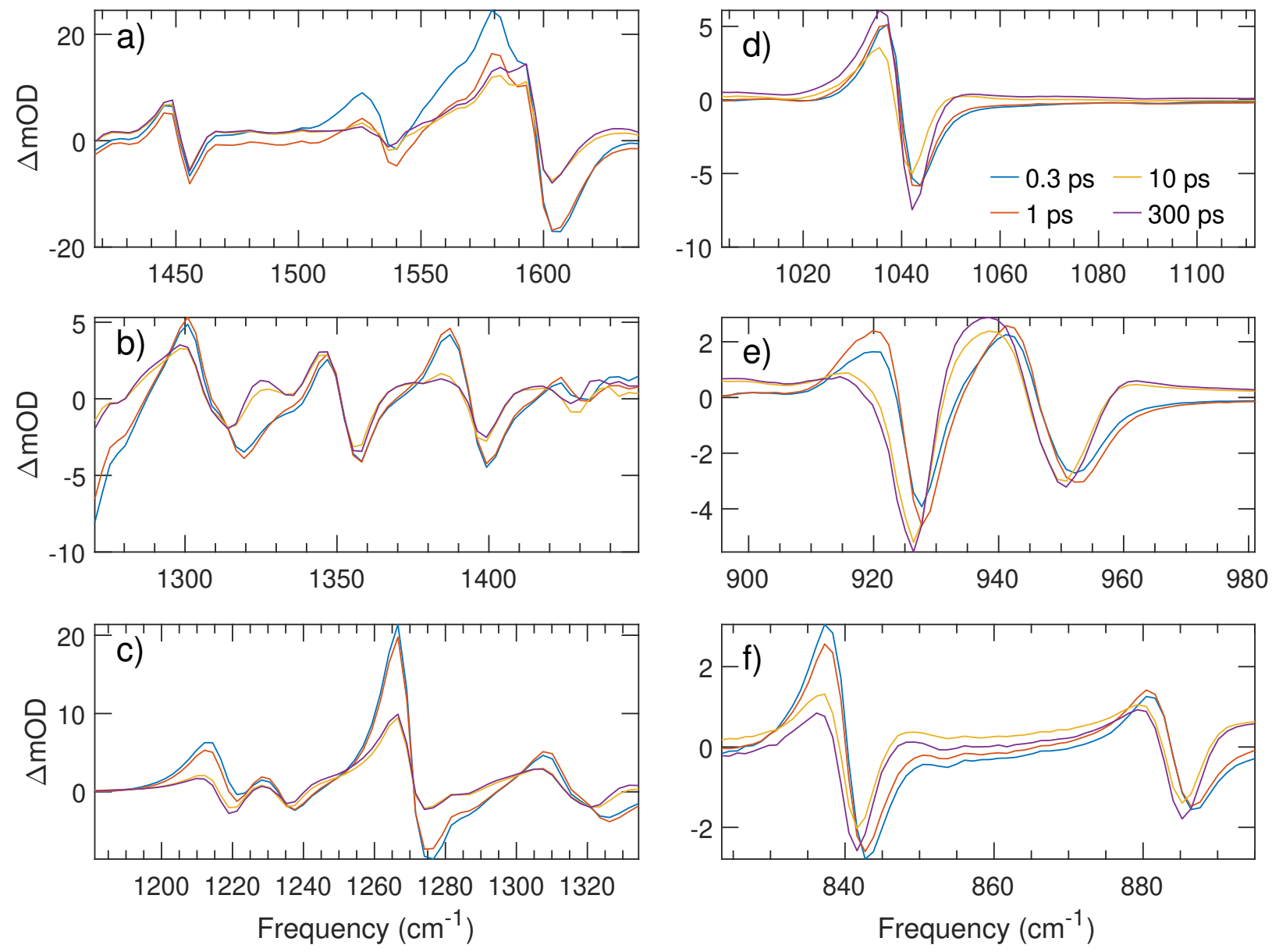

Figure S10: Transient spectra $\left(1530 \mathrm{~cm}^{-1}\right.$ pump) taken at each spectrometer grating position and multiple time delays. Spectra shown in part a) are from the transient shown in Fig. 2 following the PFID filtering process as discussed in the main manuscript. 


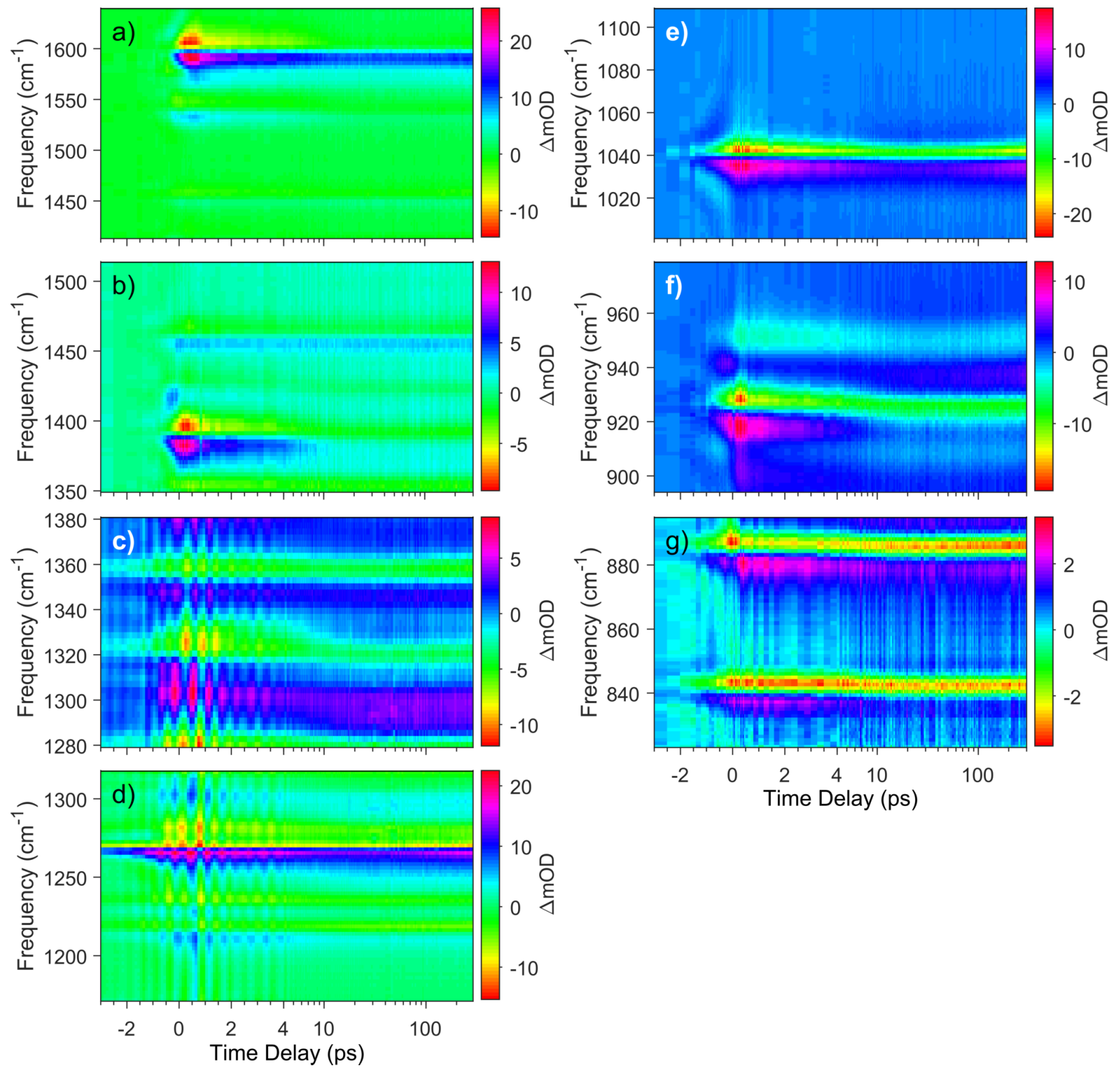

Figure S11: Reproduction of Fig. 3 (1260 $\mathrm{cm}^{-1}$ pump) using an alternate colormap; singularities are shown in white. Time-delays are shown in linear steps up to 4 ps and logarithmic thereafter. 

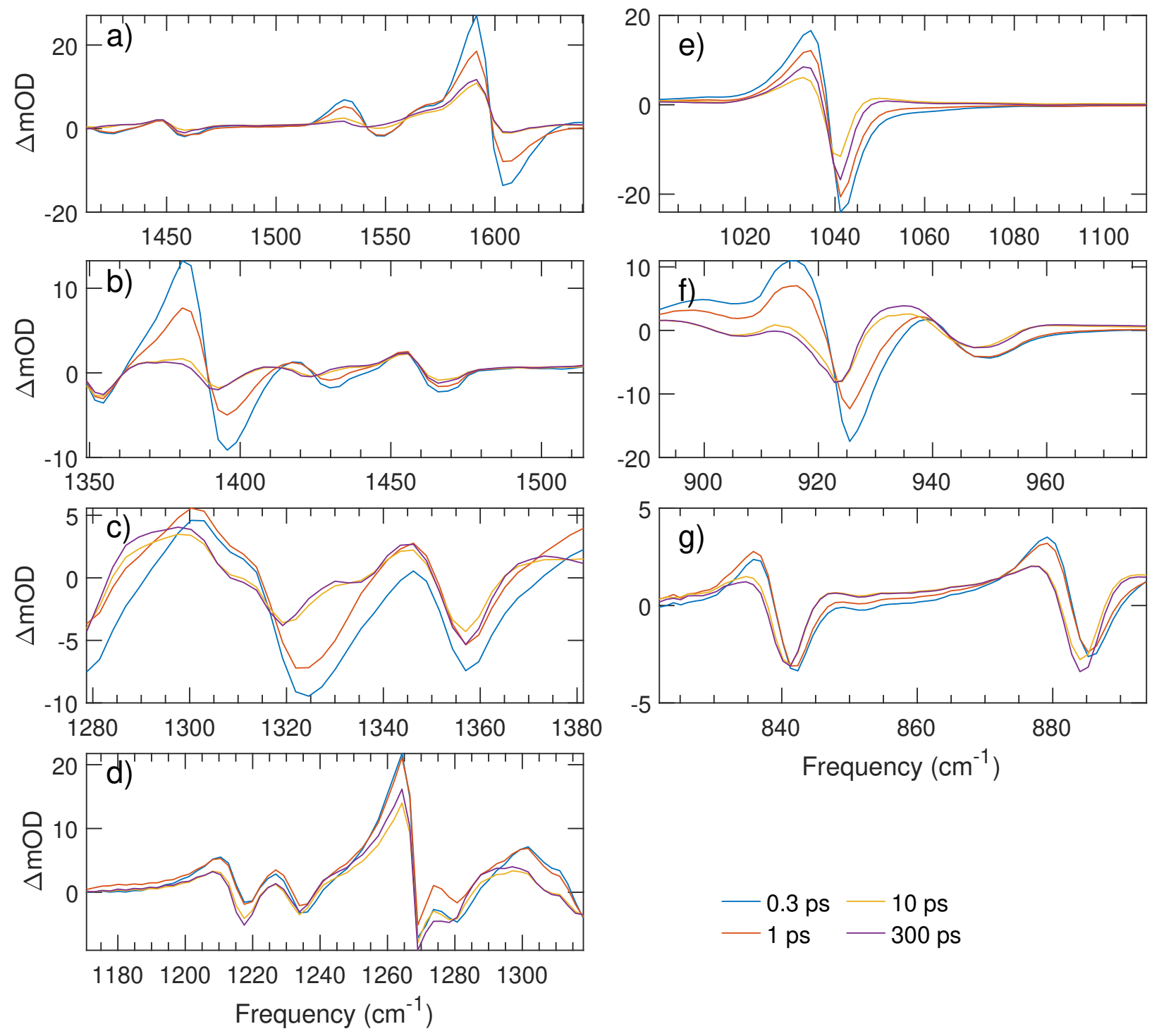

Figure S12: Transient spectra $\left(1260 \mathrm{~cm}^{-1}\right.$ pump) taken at each spectrometer grating position and multiple time delays. Spectra shown in parts c) and d) are from the transient shown in Fig. 3 following the PFID filtering process as discussed in the main manuscript. 

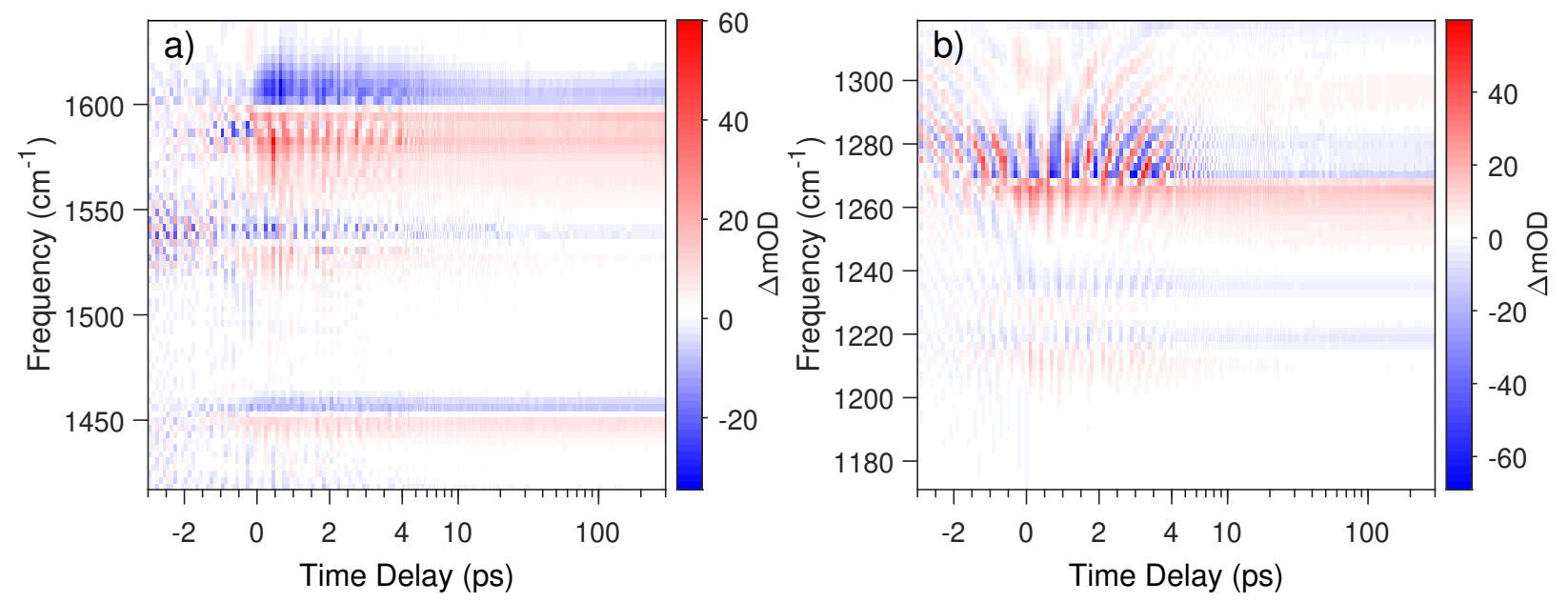

Figure S13: Transient spectra from the degerate experiments at the a) $1530 \mathrm{~cm}^{-1}$ pump and b) $1260 \mathrm{~cm}^{-1}$ pump prior to the PFID filtering process as discussed in the main manuscript.

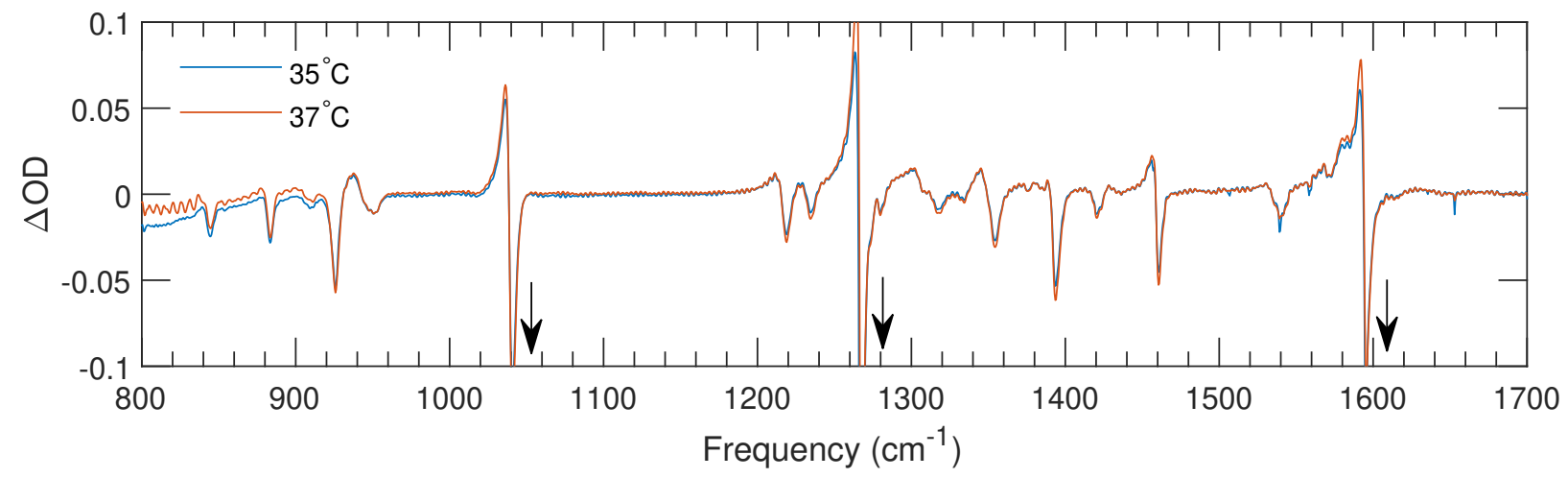

Figure S14: Thermal difference infrared spectra of RDX at $35^{\circ} \mathrm{C}$ and $37^{\circ} \mathrm{C}$. The baseline drift for frequencies $\lesssim 900 \mathrm{~cm}^{-1}$ is due to decreased transmission through the $\mathrm{CaF}_{2}$ window.

\section{S4 Kinetic Models and Fitting}

\section{S4.1 Fitting Transient Data}

For the present work, all transient data were globally fit using the Glotaran software package. ${ }^{2,3}$ In the absence of a complete kinetic picture for RDX, a sequential decay mechanism (e.g. $\mathrm{A} \rightarrow \mathrm{B} \rightarrow \mathrm{C}$ ) was assumed for all kinetic models. Fit time constants are given in Table 1 with the corresponding evolution associated difference spectra in Figs. S15 and S17; fit residuals are shown in Figs. S16 and S18.

Time delays were corrected to place $\Delta t=0$ at the position determined by an IRF measurement in InSb or Ge. Fits to each spectral window assumed an IRF of $0.3 \mathrm{ps}$ FWHM for both pump frequencies. Multiple ultrafast lifetimes were used to fit the transients near $\Delta t=0$; the shortest, sub-0.3 ps lifetime may not exclusively represent pump-induced RDX 

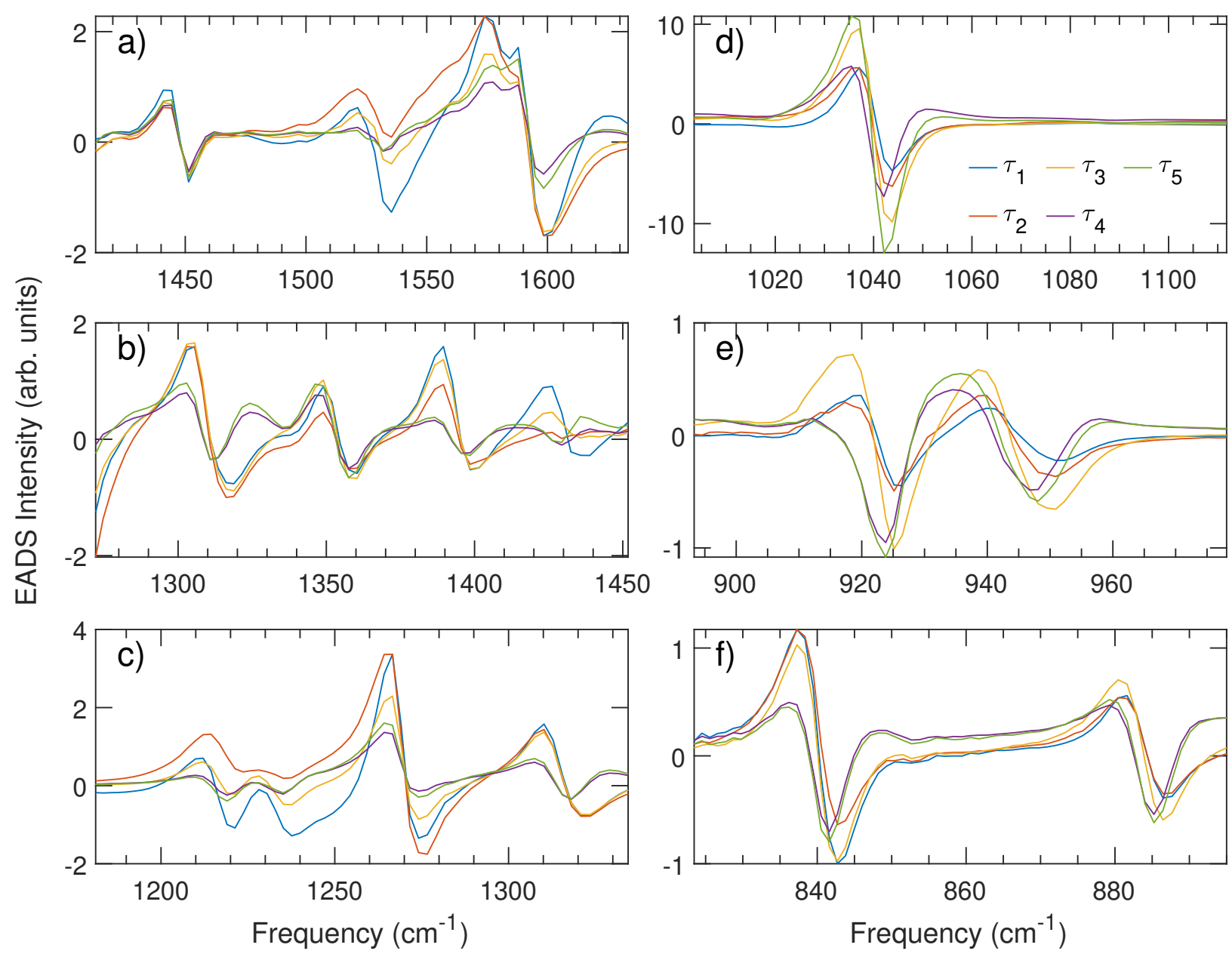

Figure S15: Evolution associated difference spectra (EADS) from global fits to the $1530 \mathrm{~cm}^{-1}$ pump transient absorption data. Time constants are given in Table 1 in the main text. EADS have been associated with $\tau_{1}$ have been scaled for visual clarity.

vibrational dynamics. Glotaran does not model dynamics at $\Delta t<0$; fits in this region will always be poor (see Figs. S16 and S18). For these data, global fitting assumes time-dependent vibrational state populations with time-independent absorption spectra. 

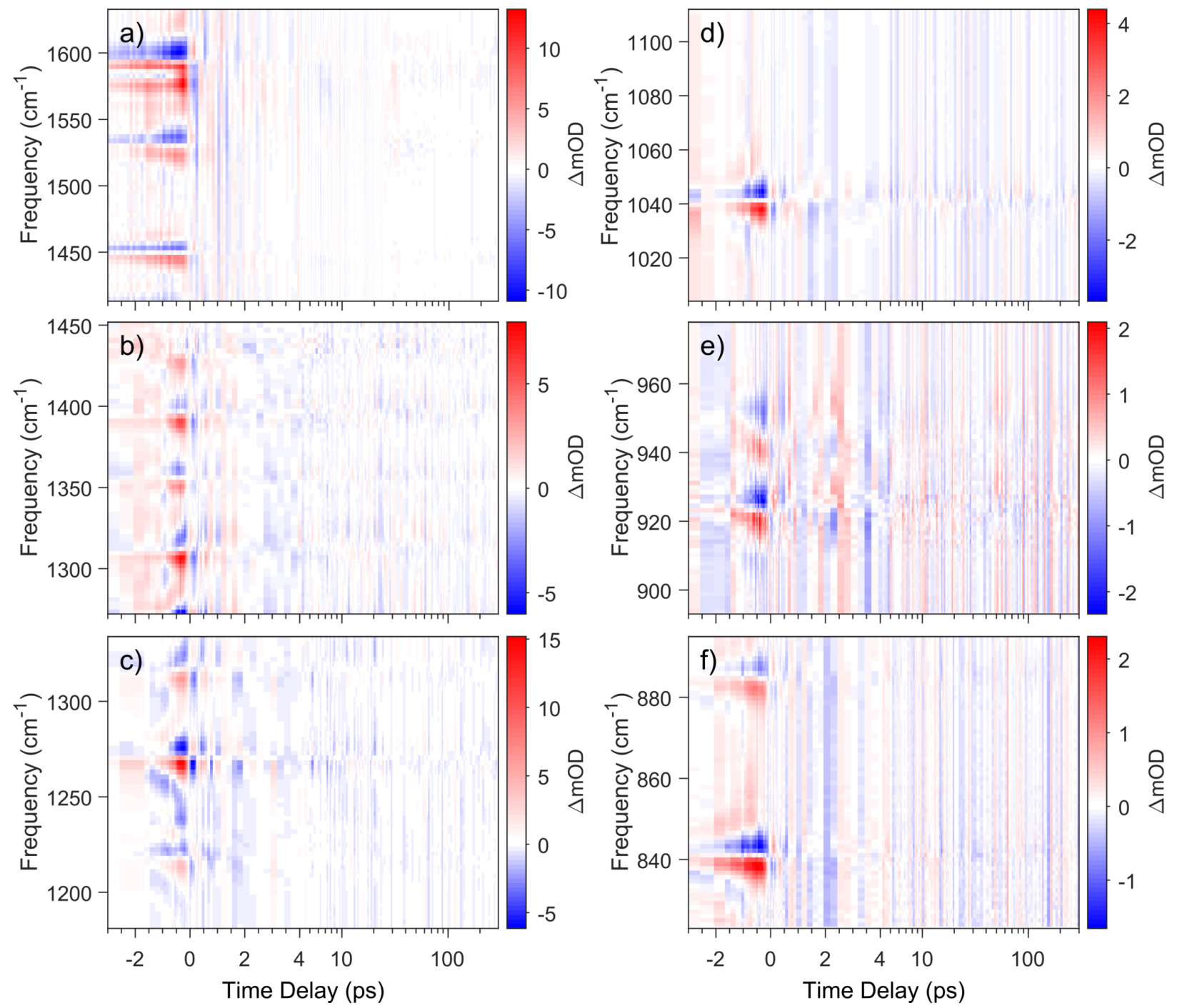

Figure S16: Fit residuals from global fits to the $1530 \mathrm{~cm}^{-1}$ pump transient absorption data using the EADS from Fig. S15 and time constants from Table 1 in the main text. 

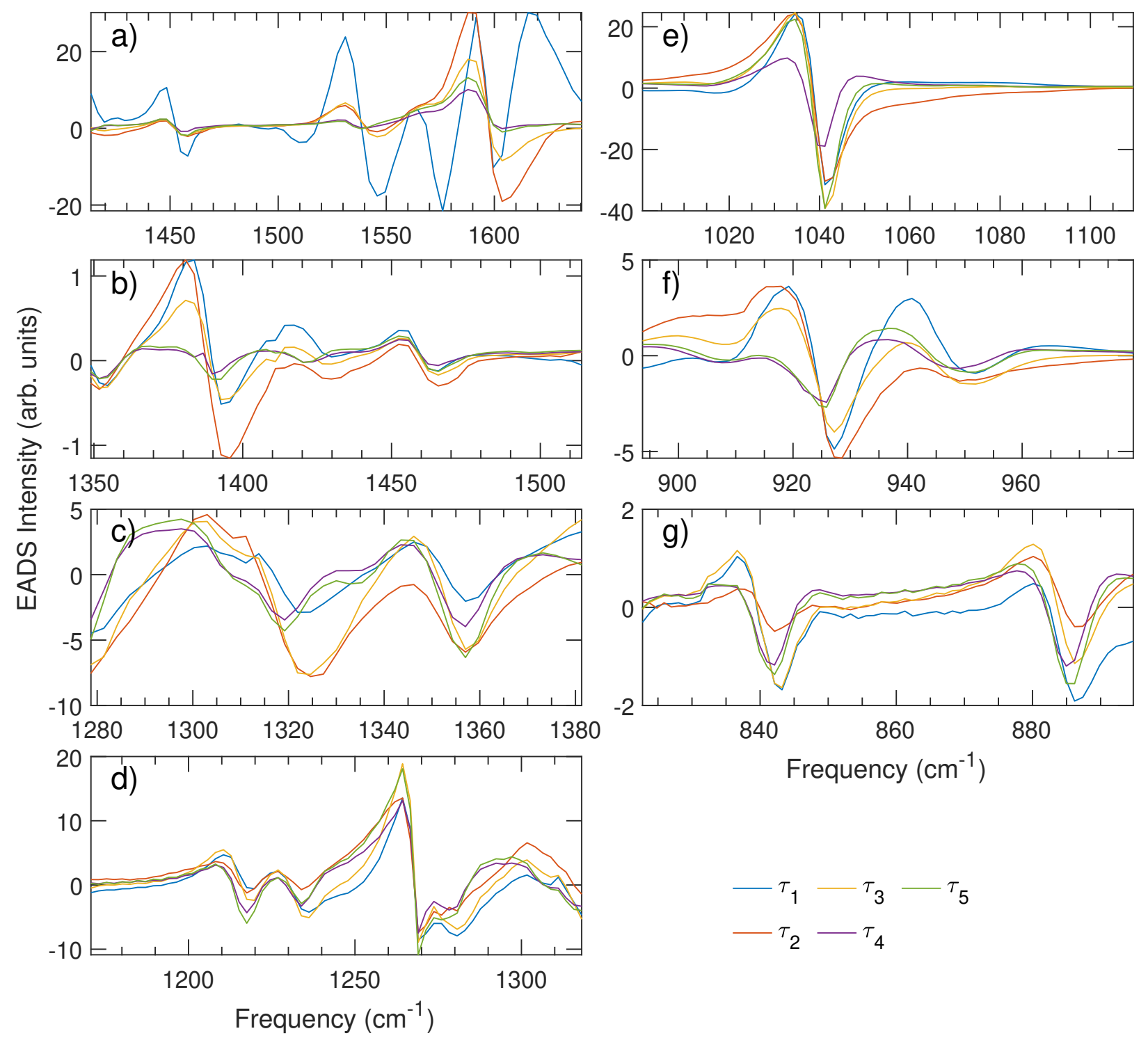

Frequency $\left(\mathrm{cm}^{-1}\right)$

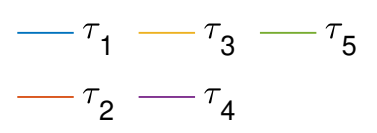

Figure S17: Evolution associated difference spectra (EADS) from global fits to the $1260 \mathrm{~cm}^{-1}$ pump transient absorption data. Time constants are given in Table 1 in the main text. EADS associated with $\tau_{1}$ have been scaled for visual clarity. 

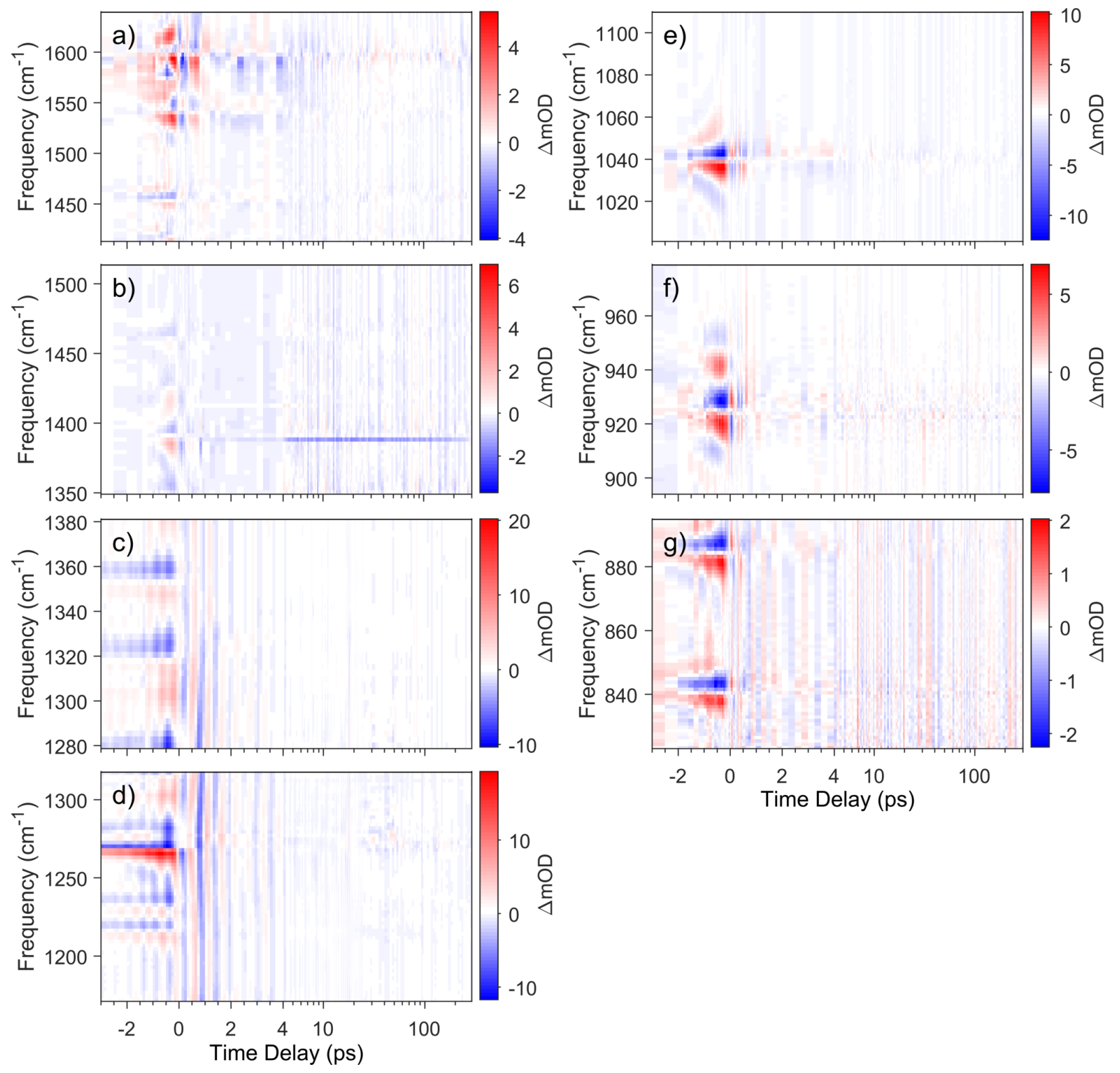

Figure S18: Fit residuals from global fits to the $1260 \mathrm{~cm}^{-1}$ pump transient absorption data using the EADS from Fig. S17 and time constants from Table 1 in the main text. 

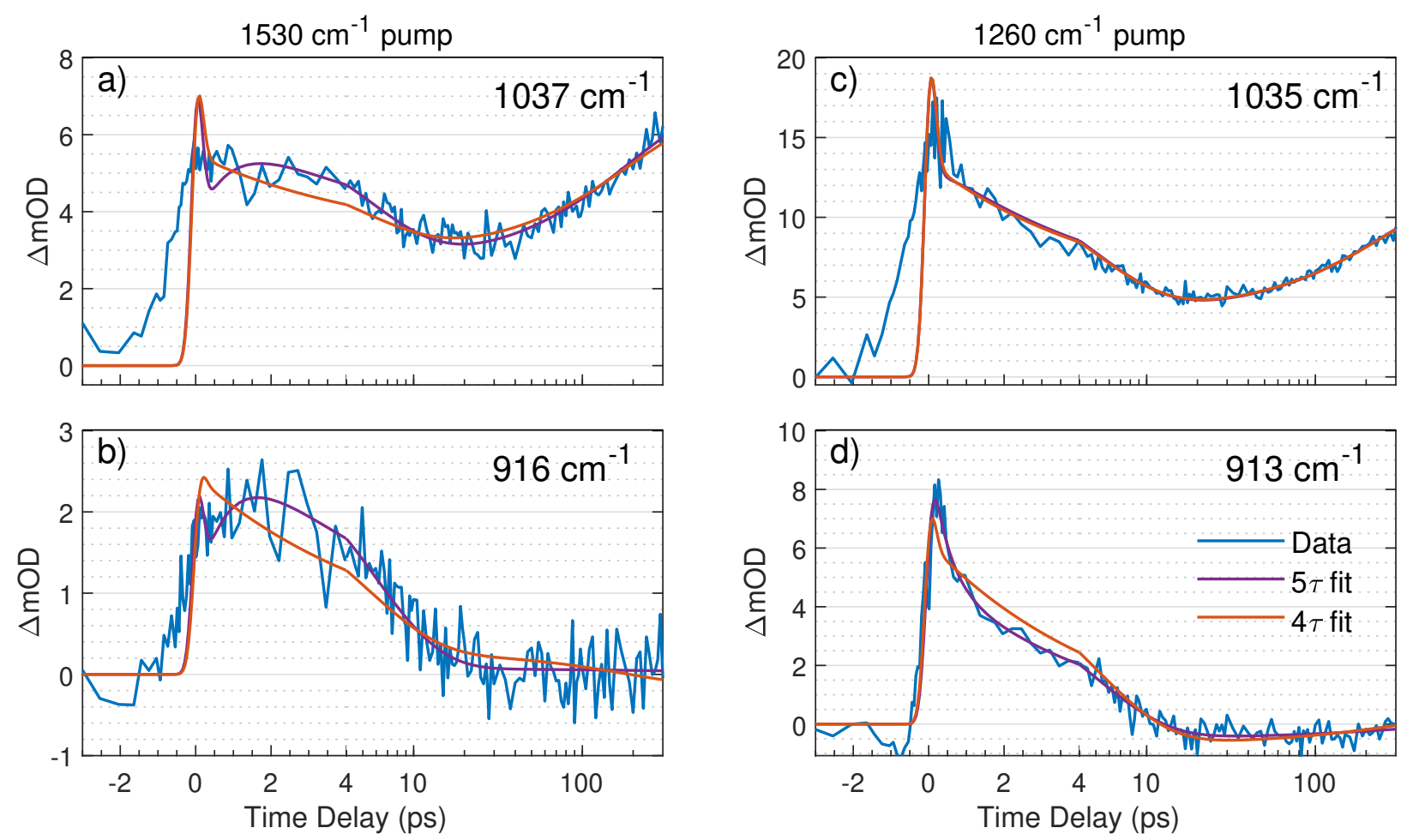

Figure S19: Transient behavior of select frequencies following excitation at $1530 \mathrm{~cm}^{-1}$ (a and b) and $1260 \mathrm{~cm}^{-1}$ (c and d). Example fits from the $5 \tau$ and $4 \tau$ kinetic models are also shown to highlight the contribution of a sub-ps time constant; see main text for further details of the fit models. Time-delays are linear up to 4 ps and logarithmic thereafter. 

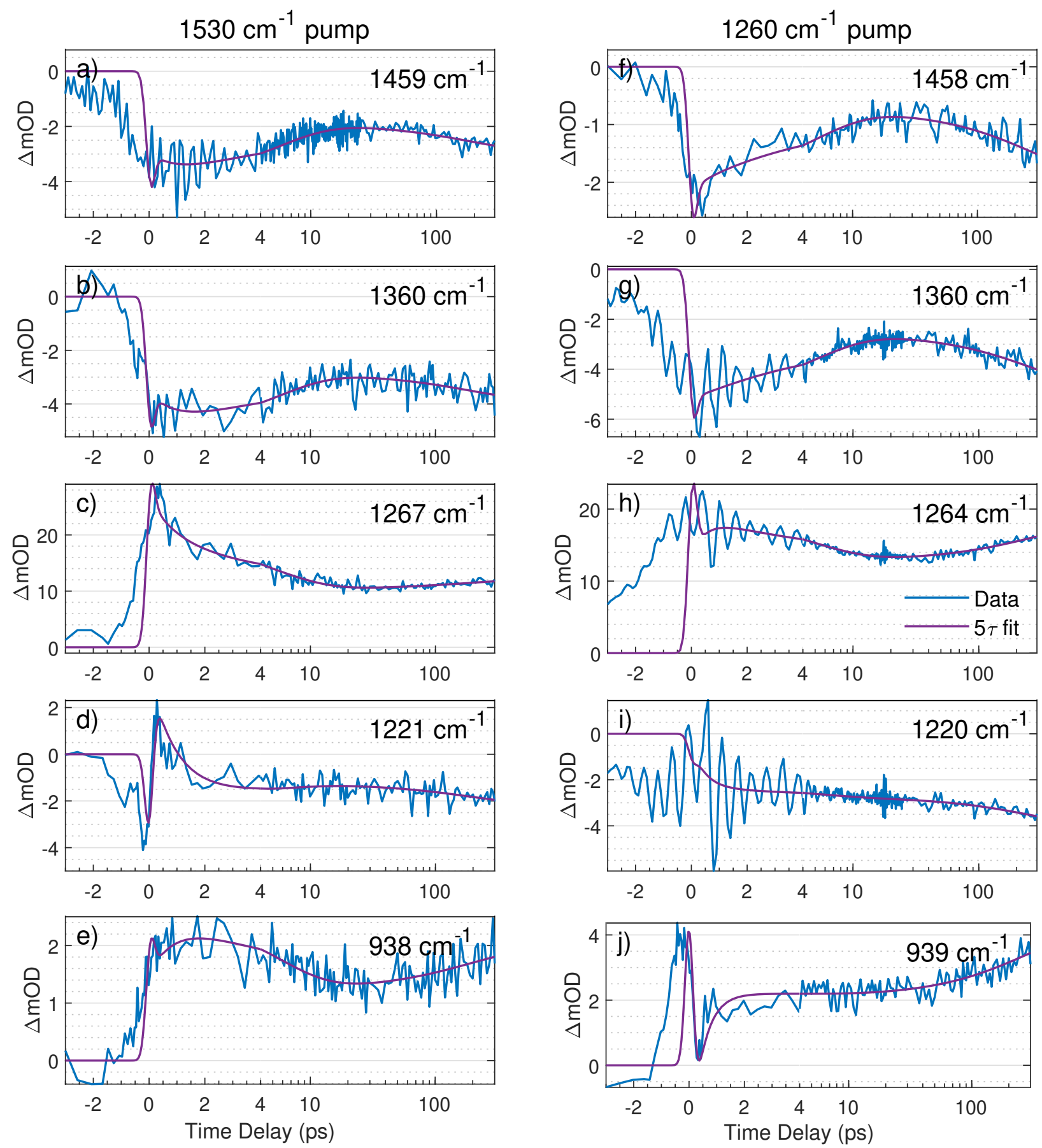

Figure S20: Additional single-frequency transients highlighting the 200 ps temporal evolution following excitation at $1530 \mathrm{~cm}^{-1}$ (left column) and $1260 \mathrm{~cm}^{-1}$ (right column). Transients at other frequencies showing 200 ps behavior are shown in Figures 5, S19, and S21. 

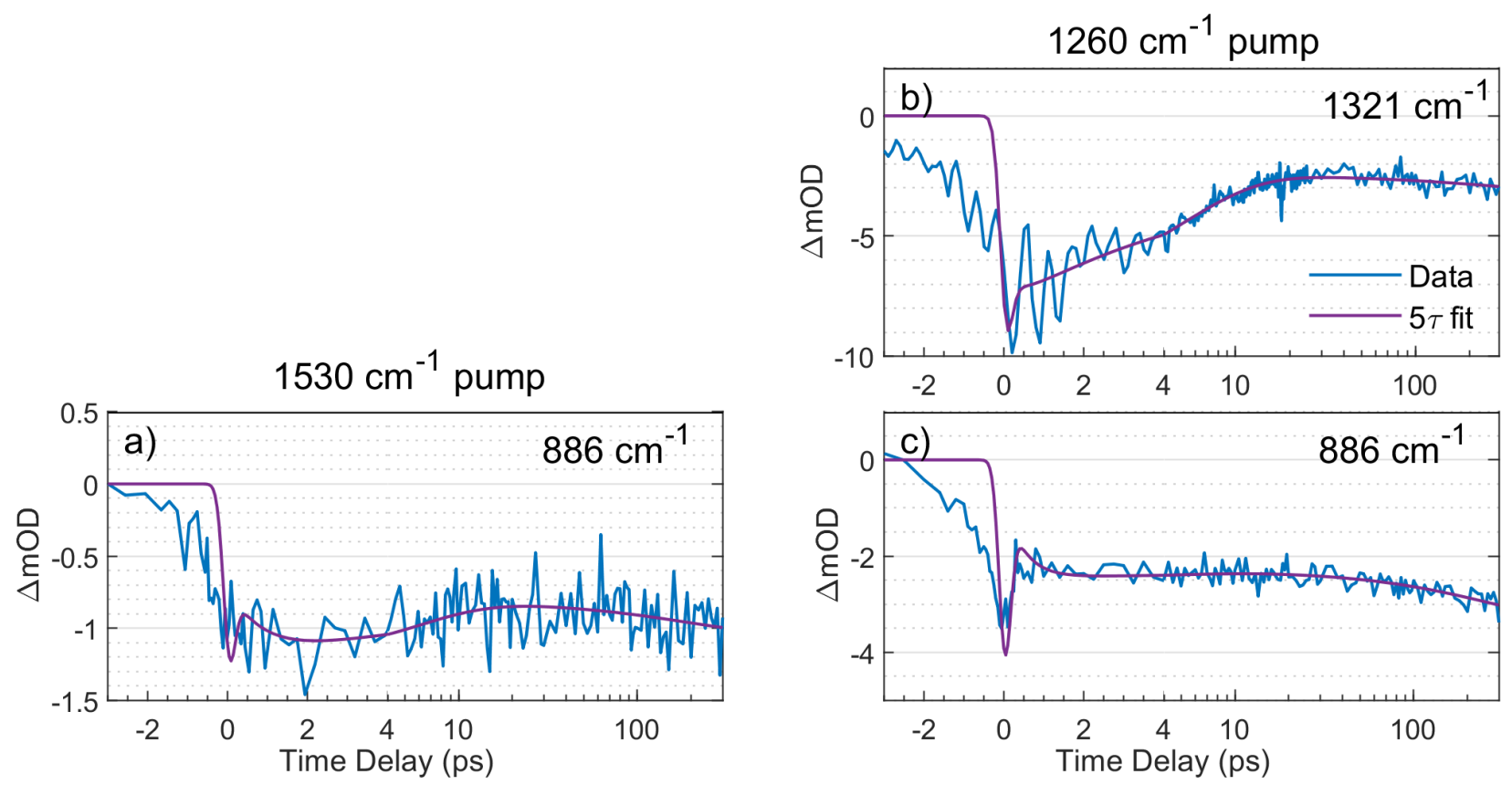

Figure S21: Additional single-frequency transients highlighting the 200 ps temporal evolution only observed following excitation at $1260 \mathrm{~cm}^{-1}$. The transient at $886 \mathrm{~cm}^{-1}$ following the $1530 \mathrm{~cm}^{-1}$ pump is also shown for signal-to-noise comparison. Transients at other frequencies showing 200 ps behavior are shown in Figures 5, S19, and S20.
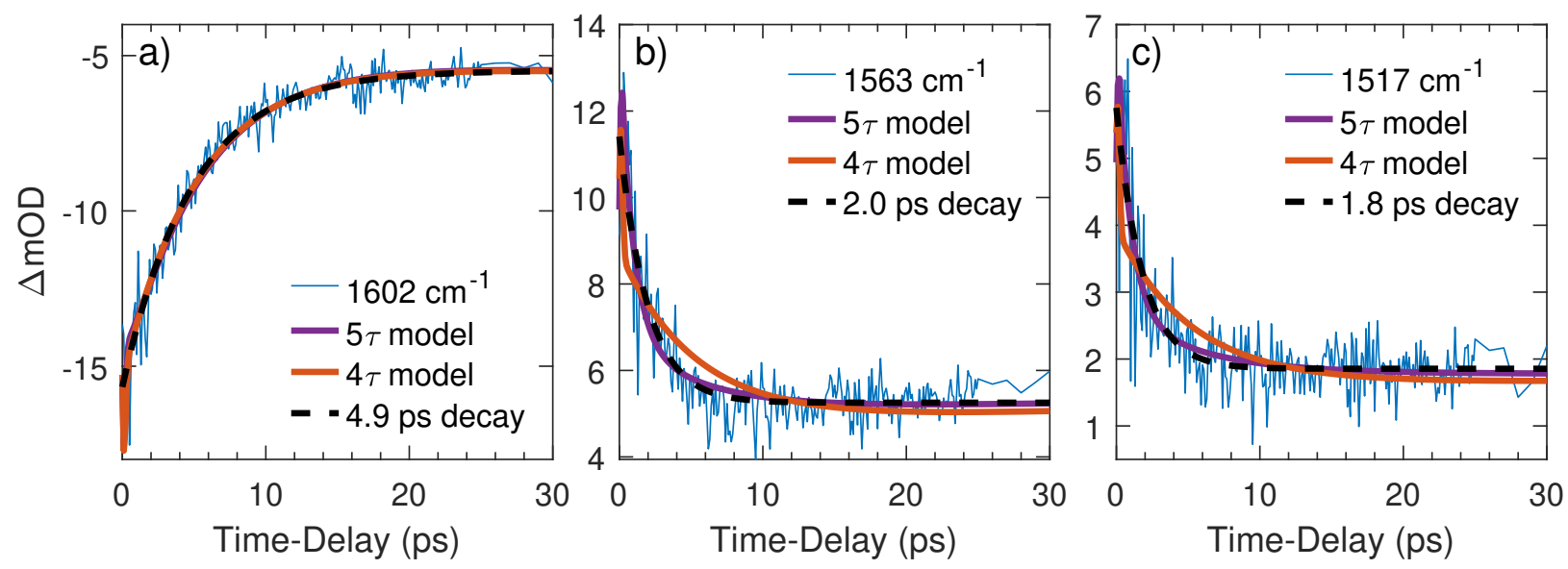

Figure S22: Transient behavior of select frequencies from the $\mathrm{NO}_{2}$ stretch modes following excitation at $1530 \mathrm{~cm}^{-1}$. For comparison to the results of Shi et al.,$^{4}$ each frequency lane $(\sim 7$ $\mathrm{cm}^{-1}$ wide) has been fit with a monoexponential decay function; time constants from these models are given in the figure legends. Example fits from the $5 \tau$ and $4 \tau$ kinetic models - as discussed in the main text - are also shown for comparison. 


\section{S5 Details of the Zenodo Archive}

Data underpinning the present results are freely available through the Zenodo data repository at doi:10.5281/zenodo.4663415.

\section{Experimental Data}

All data are saved as a .csv file. Each transient .csv file is labeled according to molecule, pump wavelength, spectrometer grating position, file contents, pump laser power, polarization, and a date (e.g. RDX8_9.5um_TA_1.4mW_para_15Sep2020.csv). The first column contains frequencies (in $\mathrm{cm}^{-1}$ ) while the first row indexes each time delay (in ps). Transient spectra at select time delays, i.e. spectra presented in Figures 4, S10, and S12, are similarly labeled (e.g. RDX8_11.65um_spec_1.6mW_para_15Sep2020.csv) with the same data organization within each file. Select transient spectra shown at the $1520 \mathrm{~cm}^{-1}\left(1530 \mathrm{~cm}^{-1}\right.$ pump) or 1330 and $1240 \mathrm{~cm}^{-1}\left(1260 \mathrm{~cm}^{-1}\right.$ pump) regions were taken from the $6.6 \mu \mathrm{m}, 7.5 \mu \mathrm{m}$, and $8 \mu \mathrm{m}$ "TA" datasets respectively. The static RDX FTIR and $35^{\circ} \mathrm{C}$ ThDIR spectra are given as two column .csv files: the first column is frequency (in $\mathrm{cm}^{-1}$ ) while the second column is either transmission or $\Delta \mathrm{OD}$.

\section{Theory Data}

Included in the data repository are all of the necessary inputs to perform the vibrational analysis from FDM using the Phonopy software package. ${ }^{5}$ The minimized RDX crystal used in the finite displacement calculations is given as a POSCAR file format to be used in VASP, the enumeration of all displacements used in the first anharmonic expansion is given in disp_fc3.yaml. The collection of all individual VASP calculations into second- and thirdorder force constants are contained in the .hdf 5 files. The remaining files contain all of the details for the Phonopy calculations done herein, per-mode population adjustments in the JDOS are achieved by modifying the phono3py/phonon3/triplets. py source code file which is distributed with all versions of the code. Additionally, individual normal mode displacements are provided as anime_*.xyz files to be read in by most visualization packages, we have used OVITO. ${ }^{6}$ To produce the displacement vector diagrams shown throughout this work, an OVITO scene file (version 3.3+) EigenModeVis_Solid_RDX.ovito has been supplied.

For the MD simulations, the modified interatomic potential and an example LAMMPS input file for a GLE pump-probe simulation are supplied. The structure file of $\alpha$-RDX has been relaxed at $1 \mathrm{~atm}$ of pressure and $300 \mathrm{~K}$ given the adjustments made to the $\mathrm{N}-\mathrm{O}, \mathrm{C}-\mathrm{H}$, $\mathrm{O}-\mathrm{N}-\mathrm{O}$, and $\mathrm{H}-\mathrm{C}-\mathrm{H}$ bond strengths. These adjustments were made based on qualitative agreements between MD and VASP predicted IR spectra, and should not be considered as a general use potential for RDX without further validation. 


\section{References}

[1] N. C. Cole-Filipiak, R. Knepper, M. Wood, and K. Ramasesha, J. Phys. Chem. Lett. 11, 6664 (2020).

[2] K. Mullen and I. van Stokkum, J. Stat. Software 18, 1 (2007).

[3] J. Snellenburg, S. Laptenok, R. Seger, K. Mullen, and I. van Stokkum, J. Stat. Software 49, 1 (2012).

[4] L. Shi, P. Yu, J. Zhao, and J. Wang, J. Phys. Chem. C 124, 2388 (2020).

[5] A. Togo and I. Tanaka, Scr. Mater. 108, 1 (2015).

[6] A. Stukowski, Modell. Simul. Mater. Sci. Eng. 18, 015012 (2009). 
